\title{
A Century of Gestalt Psychology in Visual Perception: I. Perceptual Grouping and Figure-Ground Organization
}

\author{
Johan Wagemans \\ University of Leuven (KU Leuven) and Institute of Advanced \\ Studies (IEA-Paris), Paris, France
}

\author{
Michael Kubovy \\ University of Virginia
}

Mary A. Peterson

University of Arizona

\author{
James H. Elder \\ York University \\ Stephen E. Palmer \\ University of California, Berkeley \\ Manish Singh \\ Rutgers, The State University of New Jersey-New Brunswick
}

\author{
Rüdiger von der Heydt \\ Johns Hopkins University
}

\begin{abstract}
In 1912, Max Wertheimer published his paper on phi motion, widely recognized as the start of Gestalt psychology. Because of its continued relevance in modern psychology, this centennial anniversary is an excellent opportunity to take stock of what Gestalt psychology has offered and how it has changed since its inception. We first introduce the key findings and ideas in the Berlin school of Gestalt psychology, and then briefly sketch its development, rise, and fall. Next, we discuss its empirical and conceptual problems, and indicate how they are addressed in contemporary research on perceptual grouping and figure-ground organization. In particular, we review the principles of grouping, both classical (e.g., proximity, similarity, common fate, good continuation, closure, symmetry, parallelism) and new (e.g., synchrony, common region, element and uniform connectedness), and their role in contour integration and completion. We then review classic and new image-based principles of figure-ground organization, how it is influenced by past experience and attention, and how it relates to shape and depth perception. After an integrated review of the neural mechanisms involved in contour grouping, border ownership, and figure-ground perception, we conclude by evaluating what modern vision science has offered compared to traditional Gestalt psychology, whether we can speak of a Gestalt revival, and where the remaining limitations and challenges lie. A better integration of this research tradition with the rest of vision science requires further progress regarding the conceptual and theoretical foundations of the Gestalt approach, which is the focus of a second review article.
\end{abstract}

Keywords: Gestalt, grouping principles, figure-ground organization, neural mechanisms, vision science

This article was published Online First July 30, 2012.

Johan Wagemans, Laboratory of Experimental Psychology, University of Leuven (KU Leuven), Leuven, Belgium, and Institute of Advanced Studies (IEA-Paris), Paris, France; James H. Elder, Centre for Vision Research, York University, Toronto, Ontario, Canada; Michael Kubovy, Department of Psychology, University of Virginia; Stephen E. Palmer, Department of Psychology, University of California, Berkeley; Mary A. Peterson, Department of Psychology and Cognitive Science Program, University of Arizona; Manish Singh, Department of Psychology and Center for Cognitive Science, Rutgers, The State University of New Jersey-New Brunswick; Rüdiger von der Heydt, Mind/Brain Institute, Johns Hopkins University.

Johan Wagemans was supported by the Methusalem program from the Flemish Government (METH/08/02) for a research program aimed at the integration of Gestalt psychology into modern vision science (see www .gestaltrevision.be) and by fellowships from the Research Fund (FWOFlanders) and from IEA-Paris during his sabbatical. James H. Elder was supported by NSERC, OCE, and GEOIDE. Michael Kubovy was supported by National Science Foundation (NSF) Grants BCS 1027120 and
1027259. Stephen E. Palmer was supported by NSF Grants BCS 1059088 and 0745820. Mary A. Peterson was supported by NSF Grant BCS0960529. Manish Singh was supported by National Institutes of Health (NIH) Grant EY021494 (joint with Jacob Feldman) and NSF Grant DGE 0549115 (Rutgers IGERT in Perceptual Science). Rüdiger von der Heydt was supported by NIH Grants EY02966 and EY16281 and by Office of Naval Research Grant N000141010278. Any opinions, findings, and conclusions or recommendations expressed in this material are those of the authors and do not necessarily reflect the views of the funding agencies.

We would like to thank Stephanie Poot for administrative help with the reference list, the copyrights, and the layout, as well as M. Dorothee Augustin, Wouter Braet, Peter Claessens, Maarten Demeyer, Lee deWit, Sergei Gepshtein, Frouke Hermens, Ruth Kimchi, Ervin Poljac, Peter Van der Helm, and Cees van Leeuwen for comments on previous drafts.

Correspondence concerning this article should be addressed to Johan Wagemans, University of Leuven (KU Leuven), Laboratory of Experimental Psychology, Tiensestraat 102, Box 3711, BE-3000 Leuven, Belgium. E-mail: johan.wagemans@psy.kuleuven.be 


\section{General Introduction}

Exactly 100 years ago, Wertheimer (1912) published his paper on phi motion-perception of pure motion, without object motion-which many consider to be the beginning of Gestalt psychology as an important school of thought. The present status of Gestalt psychology is ambiguous. On the one hand, many psychologists believe that the Gestalt school died with its founding fathers in the 1940s or after some devastating empirical findings regarding electrical field theory in the 1950 s, or that it declined because of fundamental limitations that blocked further progress, while stronger theoretical and experimental frameworks arose in the 1960s and 1970s that have dominated the field ever since (e.g., cognitive science, neuroscience). On the other hand, almost all psychology textbooks still contain a Gestalt-like chapter on perceptual organization (although often poorly connected to the other chapters), and new empirical papers on Gestalt phenomena appear with increasing frequency.

We are convinced that Gestalt psychology is still relevant to current psychology in several ways. First, questions regarding the emergence of structure in perceptual experience and the subjective nature of phenomenal awareness (e.g., visual illusions, perceptual switching, context effects) continue to inspire contemporary scientific research, using methods and tools that were not at the Gestaltists' disposal. Second, the revolutionary ideas of the Gestalt movement continue to challenge some of the fundamental assumptions of mainstream vision science and cognitive neuroscience (e.g., elementary building blocks, channels, modules, informationprocessing stages). Much progress has been made in the field of nonlinear dynamical systems, both theoretically and empirically (e.g., techniques to measure and analyze cortical dynamics), progress that allows modern vision scientists to surpass some of the limitations in old-school Gestalt psychology as well as those in mainstream vision research.

The centennial anniversary of Gestalt psychology is therefore an excellent opportunity to take stock of what we have discovered about core Gestalt phenomena of perceptual organization and how our understanding of the underlying mechanisms has evolved since Wertheimer's seminal contribution. Due to this review's scope, we divide it in two parts: This article deals with perceptual grouping and figure-ground organization, whereas the second covers modern developments regarding the general conceptual and theoretical frameworks that underlie Gestalt ideas (e.g., holism, emergence, dynamics, simplicity). In Table 1, we provide an overview of the topics covered in the first review article, together with the section headings, the questions or issues being raised, and some of the answers provided. One of the aims of our review is to remove the many misunderstandings surrounding Gestalt psychology, which are listed separately in Table 2, along with a more balanced view on the actual state of affairs.

To put these two reviews in perspective, we first introduce the key findings and ideas of the founders of Gestalt psychology, along with a brief sketch of its further development, rise, and fall (for an extensive treatment, see Ash, 1995). The historical section ends with a discussion of Gestalt psychology's empirical and conceptual problems and an indication of how these limitations are being addressed in current research. We then review current research on perceptual grouping and figure-ground organization in more detail in the remaining sections. We focus on these two topics because they were the most important ones in the Gestalt tradition of perceptual organization and still are today, even for vision in general.

\section{A Brief History of Gestalt Psychology}

This section addresses four questions regarding Gestalt psychology: (a) How did it start? (b) What does it stand for? (c) How did it evolve? (d) Where does it stand now?

\section{The Emergence of Gestalt Psychology}

What Max Wertheimer discovered in 1912 was called phi motion, a special case of apparent motion. (For an excellent discussion of its historical importance, see R. Sekuler, 1996; for a demonstration of the phenomenon and for a review of its misrepresentation in later sources, see Steinman, Pizlo, \& Pizlo, 2000.) According to the conventional view of apparent motion, we see an object at several successive positions and motion is then added subjectively. If this were correct, then an object would have to be seen as moving, and at least two positions - the starting and end points - would be required to produce seen motion. Neither of these conditions held in the case of phi motion. In the key experiment, a white strip was placed on a dark background in each of two slits in the wheel of a tachistoscope, and the rotation speed was adjusted to vary the time required for the light to pass from one slit to the next (i.e., the interval between the two). Above a certain threshold value $(\sim 200 \mathrm{~ms})$, observers saw the two lines in succession. With much shorter intervals $(\sim 30 \mathrm{~ms})$, the two lines appeared to flash simultaneously. At the optimal stage ( $\sim 60 \mathrm{~ms})$, observers perceived a motion that could not be distinguished from real motion. When the interval was decreased slightly below 60 $\mathrm{ms}$, after repeated exposures, observers perceived motion without a moving object - that is, pure phenomenal or phi motion. Although only three observers were tested, "the characteristic phenomena appeared in every case unequivocally, spontaneously, and compellingly" (Wertheimer, 1912/1961, p. 1042). In the same paper, Wertheimer proposed a physiological model described in terms of a short circuit and a flooding back of the current flow ("transverse functions of a special kind;" Wertheimer, 1912/1961, p. 1085), which produced what he called "a unitary continuous whole-process" (Wertheimer, 1912/1961, p. 1087). He then extended this theory to the psychology of pure simultaneity (for the perception of form or shape) and of pure succession (for the perception of rhythm or melody). These extensions were decisive for the emergence of Gestalt theory.

\section{Essentials of Gestalt Theory}

The phi phenomenon was the perception of a pure process, a transition that could not be composed from more primitive percepts of a single object at two locations. In other words, perceived motion was not added subjectively after the sensory registration of two spatiotemporal events but had its own phenomenological characteristics and ontological status. From this phenomenon, Wertheimer concluded that structured wholes or Gestalten, rather than sensations, are the primary units of mental life. This was the key idea of the new and revolutionary Gestalt theory, developed by Wertheimer and his colleagues in Berlin. An overview of how the 
Table 1

Overview of the Article With Section Numbers and Headings, Questions and Issues Raised, and Answers Provided

\begin{tabular}{|c|c|}
\hline Section number & Section title \\
\hline 1 & General Introduction \\
\hline 2 & A Brief History of Gestalt Psychology \\
\hline 2.1 & The Emergence of Gestalt Psychology \\
\hline 2.2 & Essentials of Gestalt Theory \\
\hline 2.3 & $\begin{array}{l}\text { Further Development, Rise, and Fall of Gestalt } \\
\text { Psychology }\end{array}$ \\
\hline 2.4 & The Current Status of Gestalt Psychology \\
\hline 3 & Perceptual Grouping \\
\hline 3.1 & Introduction \\
\hline 3.2 & New Principles of Grouping \\
\hline 3.3 & Grouping Principles in Discrete Static Patterns \\
\hline 3.4 & Grouping Principles in Discrete Dynamic Patterns \\
\hline 3.5 & At What Level Does Grouping Happen? \\
\hline 4 & Contour Integration and Completion \\
\hline 4.1 & Introduction \\
\hline 4.2 & Grouping Principles for Contour Integration \\
\hline 4.3 & Contour Completion \\
\hline 4.3.1 & Modal and amodal completion \\
\hline 4.3 .2 & Grouping and shape problem \\
\hline 4.3 .3 & Contour interpolation and extrapolation \\
\hline
\end{tabular}

\subsubsection{Surface geometry and layout}

4.4 Some General Issues Regarding Perceptual Grouping and Contour Integration

$\begin{array}{lll}5 & 4.5 & \text { Conclusion } \\ 5.1 & \text { Figure-Ground Organization } \\ 5.1 & \text { Introduction }\end{array}$

We explain why an extensive review of 100 year of research on perceptual organization is valuable.

We address four questions regarding Gestalt psychology:

(1) How did it start?

(2) What does it stand for?

(3) How did it evolve?

(4) Where does it stand now?

-We distinguish grouping and figure-ground organization.

-We enumerate the classic grouping principles: proximity, similarity, common fate, symmetry, parallelism, continuity, closure.

-We review progress in our understanding of perceptual grouping since the early days of Gestalt psychology; specifically:

(1) We discuss a number of additional principles that have been discovered since the initial set was described: generalized common fate, synchrony, common region, element connectedness, uniform connectedness.

(2) We demonstrate how at least some grouping principles can be measured experimentally and expressed in quantitative laws:

(a) When several orientations can be perceived based on grouping by proximity in a particular dot lattice, the outcome is determined by the relative distance alone, not by the angle between the competing organizations (affecting the global symmetry of the lattice and how it looks).

(b) When grouping by proximity and grouping by similarity are concurrently applied to the same pattern, the two principles are combined additively.

(3) We review a century of research on grouping in dynamic patterns, including Korte's laws, element and group motion in Ternus displays, space-time coupling versus space-time tradeoff.

(4) We demonstrate that grouping principles operate at multiple levels: Provisional grouping takes place at each stage of processing, possibly with feedback from higher levels to lower ones, until a final, conscious experience arises of a grouping that is consistent with the perceived structure of the 3-D environment.

We distinguish contour grouping (integration) and contour completion.

We discuss the grouping principles that play a role in contour integration: proximity, good continuation, similarity, closure, symmetry, parallelism, convexity.

We review several issues regarding contour completion; specifically:

We distinguish modal and amodal completion.

We distinguish the grouping problem and the shape problem.

-We distinguish contour interpolation and extrapolation.

-We address two questions:

(a) What geometric properties of the visible contours are used by human vision?

(b) How are these variables combined to define the shape of the contour?

We discuss the role of surface geometry and layout in contour completion.

We address the following general questions regarding perceptual grouping and contour integration:

(1) To what extent are the Gestalt laws innate or learned?

(2) How are they combined?

(3) How can they be jointly represented in accurate computational models and useful algorithms?

-We distinguish the structuralist and Gestalt positions.

-We discuss Wertheimer's criteria to demonstrate that past experience affects initial figure-ground organization. 
Table 1 (continued)

\begin{tabular}{|c|c|c|}
\hline Section number & Section title & Questions/issues/answers \\
\hline 5.2 & $\begin{array}{l}\text { Classic Image-Based Configural Principles of Figure- } \\
\text { Ground Organization }\end{array}$ & $\begin{array}{l}\text { We discuss the classic configural principles of figure-ground } \\
\text { organization: convexity, symmetry, small region, surroundedness. }\end{array}$ \\
\hline 5.3 & $\begin{array}{l}\text { New Image-Based Principles of Figure-Ground } \\
\text { Organization }\end{array}$ & $\begin{array}{l}\text { We discuss new image-based principles of figure-ground organization: } \\
\text { lower region, top-bottom polarity, extremal edges and gradient cuts, } \\
\text { edge-region grouping, articulating motion, advancing region motion, } \\
\text { contour entropy as a ground cue ( }+ \text { part salience, axiality). }\end{array}$ \\
\hline 5.4 & $\begin{array}{l}\text { Nonimage-Based Influences on Figure-Ground } \\
\text { Perception }\end{array}$ & $\begin{array}{l}\text { We discuss the evidence for nonimage-based influences on figure-ground } \\
\text { organization: past experience, attention and perceptual set. }\end{array}$ \\
\hline 5.5 & $\begin{array}{l}\text { Figure-Ground Organization in Relation to Shape and } \\
\text { Depth Perception }\end{array}$ & $\begin{array}{l}\text { We discuss how figure-ground organization relates to shape and depth } \\
\text { perception. }\end{array}$ \\
\hline 5.6 & Conclusion & \\
\hline 6 & $\begin{array}{l}\text { Neural Mechanisms in Contour Grouping, Figure- } \\
\text { Ground Organization, and Border-Ownership } \\
\text { Assignment }\end{array}$ & \\
\hline 6.1 & Introduction & $\begin{array}{l}\text {-We review the neurophysiological studies investigating the neural } \\
\text { mechanisms in contour grouping, figure-ground organization, and } \\
\text { border-ownership assignment in an integrated way. } \\
\text { - In doing so, we demonstrate how contemporary neuroscience has } \\
\text { embraced Gestalt ideas, while doing justice to Hubel and Wiesel's } \\
\text { heritage in the following three ways: }\end{array}$ \\
\hline 6.2 & Context Integration in Illusory Contours & $\begin{array}{l}\text { (1) We demonstrate how the responses of cortical neurons can depend on } \\
\text { the parameters of the stimulus in its receptive field as well as on the } \\
\text { properties of the overall configuration in the visual field. }\end{array}$ \\
\hline 6.3 & $\begin{array}{l}\text { Figure-Ground Organization and Border-Ownership } \\
\text { Assignment }\end{array}$ & $\begin{array}{l}\text { (2) We substantiate the Gestalt postulate of autonomous organization } \\
\text { processes that form primary units of perception. }\end{array}$ \\
\hline 6.4 & Involuntary Organization and Volitional Attention & $\begin{array}{l}\text { (3) We refine our understanding about the role of attention in these } \\
\text { processes of perceptual organization. }\end{array}$ \\
\hline 6.5 & Conclusion & \\
\hline 7 & General Discussion and Conclusion & \\
\hline 7.1 & The Swinging Pendulum of Gestalt History & \\
\hline 7.2 & Gestalt Research Anno 2012 & \\
\hline 7.3 & $\begin{array}{l}\text { Limitations and Challenges to Contemporary Research } \\
\text { on Perceptual Organization }\end{array}$ & \\
\hline 7.4 & Conclusion & \\
\hline
\end{tabular}

Berlin school of Gestalt psychology distinguished itself from the dominant view of structuralism and empiricism, as well as of related Gestalt schools, is given in Table 3.

The notion of Gestalt had already been introduced into psychology by Christian von Ehrenfels in his essay "On Gestalt Qualities" (1890/1988). Based on the observation that humans can recognize two melodies as identical even when no two corresponding notes in them have the same frequency, von Ehrenfels argued that these forms must possess a "Gestalt quality" - a characteristic that is immediately given, along with the elementary sensations that serve as its foundation, a characteristic that is dependent on its constituent objects but rises above them. For von Ehrenfels, Gestalt qualities rest unidirectionally on sense data: Wholes are more than the sums of their parts, but the parts are the foundation (Grundlage) of the whole. In contrast, Wertheimer claimed that functional relations determine what will appear as the whole and what will appear as parts (i.e., reciprocal dependency). Often the whole is grasped even before the individual parts enter consciousness. The contents of our awareness are by and large not additive but possess a characteristic coherence. They are structures that are segregated from the background, often with an inner center, to which the other parts are related hierarchically. Such structures or Gestalten are different from the sum of the parts. They arise from continuous global processes in the brain, rather than combinations of elementary excitations.
With this step, Wertheimer separated himself from the Graz school of Gestalt psychology, represented by Alexius Meinong, Christian von Ehrenfels, and Vittorio Benussi. They maintained a distinction between sensation and perception, the latter produced on the basis of the former. The Berlin school, represented by Max Wertheimer, Kurt Koffka, and Wolfgang Köhler, considered a Gestalt as a whole in itself, not founded on any more elementary objects. In their view, perception was not the product of sensations but arose through dynamic physical processes in the brain. As a result, the Berlin school also rejected stage theories of perception proposed by the Leipzig school, represented by Felix Krüger and Friedrich Sander, in which the gradual emergence of Gestalten (Aktualgenese or microgenesis) played a central role. Although the Berlin theorists adhered to a nonmechanistic theory of causation and did not analyze the processes into stages, they did believe that the functional relations in the emergence of Gestalts could be specified by laws of perceptual organization.

\section{Further Development, Rise, and Fall of Gestalt Psychology}

Two major developments are generally considered as highlights in the history of Gestalt psychology: Köhler's discussion of "physical Gestalten" (1920/1938) and Wertheimer's proposal of "Gestalt laws of perceptual organization" (1923/1938c). Köhler extended 
Table 2

Common Misunderstandings About Gestalt Psychology

Gestalt psychology is completely dead and buried because its limitations have never been overcome.

Gestalt psychology was a radical, simpleminded theory that has been rejected.

All fundamental issues pertaining to perceptual grouping and figure-ground organization are solved.

\section{A. General}

Interesting work in the Gestalt tradition is still being carried out and many limitations and shortcomings have been overcome or addressed (see

Many of the ideas of Gestalt psychology are still very much alive. A century of research has allowed several more synthetic positions, integrating some of the original Gestalt positions with alternative positions (see

Important problems regarding perceptual grouping and figure-ground organizations are still unsolved. Some of these are mentioned in the course of the discussion in this article. There are still some controversial issues and open questions that continue to stimulate contemporary research. A number of challenges are listed separately in the final section of this article (see

\section{B. Specific}

Grouping principles are mere textbook curiosities only distantly related to normal perception.

Gestalt psychology has claimed that all Gestalt laws are innate and that learning or past experience can never play a role.

The Gestalt theory about brain function is rejected by the empirical evidence.

Vague Gestalt notions about whole-processes in the brain are now completely replaced by precise single-cell recordings demonstrating that neurons operate like primitive detectors.
Grouping principles pervade virtually all perceptual experiences because they determine the objects and parts we perceive in the environment.

Gestalt psychology has emphasized the autonomy of the Gestalt laws but it has not claimed that all Gestalt laws are innate and that learning or past experience can never play a role.

Köhler's specific conjecture about electromagnetic brain fields appears to be rejected by experiments by Lashley and Sperry, but advances in neurophysiology have confirmed the existence of preattentive mechanisms of visual organization postulated by Gestalt theory. The more abstract notion of the brain as a physical Gestalt can also be implemented as recurrent networks with closed feedback loops, which can be proven to converge to an equilibrium state of minimum energy.

Neurophysiology has come a long way since Hubel and Wiesel's atomistic approach to orientation-selectivity of single cells in cat and monkey cortex, taken as prototypical feature detectors. The current literature emphasizes the role of contextsensitive, autonomous processes within recurrent networks. the Gestalt concept from perception and behavior to the physical world, thus attempting to unify holism (i.e., the doctrine stressing the importance of the whole) and natural science. He proposed to treat the neurophysiological processes underlying Gestalt phenomena in terms of the physics of field continua rather than that of particles or point-masses. In such continuous field systems, which he called strong Gestalten, the mutual dependence among the parts is so great that no displacement or change of state can occur without influencing all the other parts of the system. Köhler showed that stationary electric currents, heat currents, and all phenomena of flow are strong Gestalten in this sense. These he distinguished from what he called weak Gestalten, which do not show this mutual interdependence.

In addition, Köhler (1920) tried to construct a specific testable theory of brain processes that could account for perceived Gestalten in vision. He thought of visual Gestalten as the result of an integrated process in what he referred to as "the entire optical sector" (Köhler, 1920/1938, p. 211), including retina, optical tract, and cortical areas, as well as transverse functional connections among conducting nerve fibers (i.e., a recurrent neural network in modern terms). He proposed an electrical field theory, in which "the lines of flow are free to follow different paths within the homogeneous conducting system, and the place where a given line of flow will end in the central field is determined in every case by the conditions in the system as a whole" (Köhler, 1920/1938, p. 50. In modern terms, Köhler had described the visual system as a self-organizing physical system.

These ideas led Köhler to postulate a psychophysical isomorphism between the psychological reality and the brain events underlying it: "Actual consciousness resembles in each case the real structural properties of the corresponding psychophysiological process" (Köhler, 1920/1938, p. 38). By this he meant functional instead of geometrical similarity indicating that brain processes do not take the form of the perceived objects themselves. In addition, he insisted that such a view does not prescribe complete homogeneity of the cortex but is perfectly compatible with functional articulation. Experiments to establish the postulated connections between experienced and physical Gestalten in the brain were at the time nearly impossible to conduct, but decades later, Köhler attempted to do so (see below).

Around the same time, Max Wertheimer (1922) further developed his Gestalt epistemology and outlined the research practice of experimental phenomenology that was based on it. He first stated the principles publically in a manifesto published in Volume 1 of Psychologische Forschung in 1922. Wertheimer called for descriptions of conscious experience in terms of the units people naturally perceive, rather than the artificial ones imposed by standard scientific methods. By assuming that conscious experience is composed of units analogous to physical point-masses or chemical elements, psychologists constrain themselves to a piecemeal inquiry into the contents of consciousness, building up higher entities from constituent elements, using associative connections. In fact, such and-summations (Und-Summe), as Wertheimer called them, appear "only rarely, only under certain characteristic conditions, only within very narrow limits, and perhaps never more than approximately" (Wertheimer, 1922/1938a, p. 13). Rather, what is given in experience "is itself in varying degrees 'structured' ('gestaltet'), it consists of more or less definitely structured wholes and 
Table 3

Key Claims by the Berlin School of Gestalt Psychology in Opposition to Other Schools

Berlin school of Gestalt psychology

(Wertheimer, Köhler, Koffka)
Opposing schools
Structured wholes or Gestalten are the primary units of mental life.

Experimental phenomenology: Perceptual experience must be described in terms of the units people naturally perceive.

Percepts arise on the basis of continuous whole-processes in the brain; percepts organize themselves by mutual interactions in the brain.

Perceptual organization is based on innate, intrinsic, autonomous laws.

Simplicity or minimum principle.

Gestalten (structured experiences, wholes) are different from the sum of the parts.

Two-sided or reciprocal dependency between parts and wholes:

- There are specifiable functional relations that decide what will appear or function as a whole and what as parts.

-Often the whole is grasped even before the individual parts enter consciousness.

Perception "emerges" through self-organization; perception arises nonmechanistically through an autonomous process in the brain.

No analysis into stages, but functional relations in the emergence of Gestalts can be specified by Gestalt laws of perceptual organization.

Holism integrated with natural science (physical Gestalten, isomorphism, minimum principle).
Structuralism/associationism/empiricism (von Helmholtz, Wundt)

Sensations are the primary units of mental life.

Introspection: Perceptual experience must be analyzed as combinations of elementary sensations of physical stimuli as their building blocks.

Percepts are associated combinations of elementary excitations.

Perceptual organization is based on perceptual learning, past experience, intentions.

Likelihood principle

Graz school of Gestalt psychology (Meinong; von Ehrenfels, Benussi)

Gestalt qualities are more than the sum of the constituent primary sensations.

One-sided dependency between parts and wholes (the wholes depend on the parts, but the parts do not depend on the whole).

Perception is "produced" on the basis of sensations.

Leipzig school of Gestalt psychology (Krüger, Sander) Stage theory: Aktualgenese, microgenesis.

Mystic holism, segregated from natural science. whole-processes with their whole-properties and laws, characteristic whole-tendencies and whole-determinations of parts" (Wertheimer, 1922/1938a, p. 14). The perceptual field does not appear to us as a collection of disjointed sensations, but possesses a particular organization of spontaneously combined and segregated objects.

In 1923, Wertheimer published a follow-up paper, which was an attempt to elucidate the fundamental principles of that organization. The most general principle was the so-called law of Prägnanz, stating, in its most general sense, that the perceptual field and objects within it will take on the simplest and most encompassing (ausgezeichnet) structure permitted by the given conditions. For Köhler (1920), this tendency towards the Prägnanz of the Gestalt was just another example that phenomenal Gestalten were like physical Gestalten: As shown by Maxwell and Planck, all processes in physical systems, left to themselves, show a tendency to achieve the maximal level of stability (homogeneity, simplicity, symmetry) with the minimum expenditure of energy allowed by the prevailing conditions. More specific principles that determine perceptual organization according to Wertheimer were proximity, similarity, uniform density, common fate, direction, good continuation and whole properties (or Ganzeigenschaften) such as closure, equilibrium, and symmetry.

Empirical work on these principles existed before Wertheimer's landmark paper (for a recent review, see Vezzani, Marino, \& Giora, 2012), but now the general claim that perceptual experience is organized was turned into a complex open-ended research program aimed at the discovery of the laws or principles governing perceptual organization in both its static and dynamic aspects. It is this research program that Wertheimer, Koffka, and Köhler started to work on with their students, once they had acquired professorships at major universities in Germany in the 1920s and 1930s. We cannot cover this flourishing period of the Berlin school of Gestalt psychology extensively here, but a few highlights that deserve mentioning in passing are studies by Kurt Gottschaldt on embedded figures (1926), Joseph Ternus on phenomenal identity (1926), Karl Duncker on induced motion (1929), Wolfgang Metzger on a homogeneous Ganzfeld (1930) and motion in depth (1934). In the meantime, Gestalt thinking also affected research on other sense modalities (e.g., binaural hearing by Erich von Hornbostel), on learning and memory (e.g., Otto von Lauenstein and Hedwig von Restorff), and on thought (e.g., Karl Duncker). Later, Gestalt theory was also applied to action and emotion (by Kurt Lewin), to neuropathology and the organism as a whole (by Adhemar Gelb and Kurt Goldstein), and to film theory and aesthetics (by Rudolf Arnheim). This period marked the high point but not the end of Gestalt psychology's theoretical development, its research productivity, and its impact on German science and culture.

Around this time, Gestalt theory also started to have some impact on research in the United States, mainly owing to Wolfgang Köhler and Kurt Koffka (see King \& Wertheimer, 2005, Chapter 10). For instance, Koffka's (1935) notion of vector fields inspired some interesting empirical work published in the American Journal of Psychology (Brown \& Voth, 1937; Orbison, 1939). Reviews 
of Gestalt psychology appeared in Psychological Review on a regular basis (e.g., Helson, 1933; Hsiao, 1928), a comprehensive book on state-of-the-art Gestalt psychology was published as early as 1935 (Hartmann, 1935), and 3 years later Ellis's (1938) influential collection of translated excerpts of core Gestalt readings made some of the original sources accessible to a non-Germanspeaking audience. Already in 1922, at Robert Ogden's invitation, Koffka had published a full account of the Gestalt view on perception in Psychological Bulletin.

At first sight, Gestalt theory seemed to develop rather consistently, from studying the fundamental laws of psychology first under the simplest conditions, in elementary problems of perception, before including complex sets of conditions, and turning to other domains such as memory, thinking, emotion, aesthetics, and so forth. At the same time, however, the findings obtained did not always fit the original theories, which posed serious challenges to the Gestalt framework. Even more devastating to the development of Gestalt psychology was the emergence of the Nazi regime in Germany from 1933 to World War II. In this period, many of the psychology professors at German universities lost their posts because of the discrimination against and prosecution of Jews, so they emigrated to the United States to take on new positions there. The works by German psychologists who stayed, for instance, Edwin Rausch's monograph on "summative" and "nonsummative" concepts (1937) and Wolfgang Metzger's (1941) psychology textbook, were largely ignored outside Germany. Metzger's synoptic account of research on the Gestalt theory of perception entitled Gesetze des Sehens (Laws of Seeing), first published in 1936 and later reissued and vastly expanded three times, was only translated into English in 2006.

After emigrating to the United States, the founding fathers of Gestalt psychology did not perform many new experiments. Instead, they mainly wrote books in which they outlined their views (e.g., Koffka, 1935; Köhler, 1940; Wertheimer, 1945). The major exception was Köhler who had taken up physiological psychology using electroencephalograph (EEG) recording and other methods in an attempt to directly verify his isomorphism postulate. Initially, his work with Hans Wallach on figural aftereffects appeared to support his interpretation in terms of satiation of cortical currents (Köhler \& Wallach, 1944). Afterwards, he was able to directly measure cortical currents-as EEG responses picked up from electrodes at the scalp—whose flow direction corresponded to the direction of movement of objects in the visual field (Köhler \& Held, 1949).

Soon after that breakthrough, however, Lashley, Chow, and Semmes (1951) performed a more critical test of Köhler's electric field theory and its underlying postulate of isomorphism. If the flows of current picked up from the scalp in Köhler and Held's experiments indeed reflected the organized pattern of perception and not merely the applied stimulation, and if that pattern of perception would result from a global figure-field across the whole cortex, a marked alteration of the currents should distort the perception of these visual figures. By inserting metallic strips and metal pins in large regions of the visual cortex of rhesus monkeys, Lashley et al. could short-circuit the cortical currents. Surprisingly, the monkeys could still perform the learned shape discriminations, demonstrating that global cortical currents were not a necessary condition for pattern perception. In subsequent experiments, Sperry, Miner, and Myers (1955) performed extensive slicing and dense impregnation with metallic wires across the entire visual cortex of cats, and showed that these animals too could still perform rather difficult shape discriminations (e.g., between a prototypical triangle and distorted variants). Together, these two studies effectively ruled out electrical field theory as an explanation of cortical integration and undermined the empirical basis of any isomorphism between cortical flows of current and organized patterns of perception. Köhler (1965) naturally reacted to these developments but his counterarguments and suggestions for further experiments were largely ignored, and to most scientists at the time, the matter was closed. Electrical field theory, which had been one of the pillars of Gestalt psychology's scientific basis, was considered dead and buried.

While Gestalt psychology declined in the English-speaking world after World War II, Italy remained a stronghold of Gestalt psychology. For instance, Metzger dedicated the third edition of his Gesetze des Sehens to his "Italian and Japanese friends." Among his friends were Musatti, Metelli, and Kanizsa-three major figures in Italian psychology. In spite of being Benussi's student and successor (from the Graz school), Cesare Musatti was responsible for introducing the Berlin school of Gestalt psychology in Italy and training important students in this tradition-most notably Metelli and Kanizsa, whose contributions continue to be felt today. Fabio Metelli is best known for his work on the perception of transparency (e.g., Metelli, 1974). Gaetano Kanizsa's most famous studies were performed in the 1950s with papers on subjective contours (e.g., the so-called Kanizsa triangle), modes of color appearance, and phenomenal transparency (Kanizsa, 1954, 1955a, 1955b), although their impact came much later, when he started to publish in English (Kanizsa, 1976, 1979).

In addition to Italy, Gestalt psychology was also strong in Belgium and in Japan. Albert Michotte became famous for his work on the perception of causality (1946/1963), arguing strongly against an inferential, associationist, empiricist account of it, like other Gestalt psychologists had done for other aspects of perception. For him, causality is perceived directly, not derived from more primitive sensations through some cognitive operation, and this percept could be shown to be tightly coupled to specific higher order attributes in the spatiotemporal events presented to observers. He also introduced the notions of modal and amodal completion (Michotte, Thinès, \& Crabbé, 1964), and studied several configural influences on these processes. (For a further discussion of Michotte's heritage, see Wagemans, van Lier, \& Scholl, 2006.) Building on earlier collaborations of Japanese students with major German Gestalt psychologists (e.g., Sakuma with Lewin, Morinaga with Metzger), Gestalt psychology continued to develop in Japan after World War II. For instance, Oyama did significant work on figural aftereffects (e.g., Sagara \& Oyama, 1957) and perceptual grouping (e.g., Oyama, 1961).

\section{The Current Status of Gestalt Psychology}

Despite signs of well-deserved respect in the United States and in Germany (e.g., Köhler's American Psychological Association presidency in 1957, Wertheimer's posthumous Wilhelm Wundt Medal in 1983), the ideas of the Gestaltists were received with ambivalence. On the one hand, they were recognized for raising central issues and provoking important debates in psychology, theoretical biology, and other fields, but on the other hand, their 
mode of thinking and research style did not sit comfortably in the intellectual and social climate of the postwar world, and they were confronted with vehement criticism. Two sets of explanations have been given for this outcome (Ash, 1995). The first emphasizes institutional, political, and biographical contingencies. Koffka, Köhler, and Wertheimer all left for the United States and obtained positions where they could do excellent research but could not train doctoral students. The Gestalt school's further expansion was also handicapped by the early deaths of Max Wertheimer in 1943 and Kurt Koffka in 1941, as well as many other Gestalt psychologists of the first and second generations (e.g., Duncker, Gelb, Lauenstein, Lewin, von Restorff). In Germany, Metzger, Rausch, and Gottschaldt did have a large number of doctoral students, but few of them carried on in the Gestalt tradition. A notable exception is Lothar Spillmann, who obtained his doctorate with Metzger in Münster in 1964 and who has pioneered the impact of Gestalt ideas in modern neurophysiology ever since (e.g., Spillmann, 1999, 2009).

The second set of explanations concerns scientific issues of a methodological and conceptual nature (summarized in the left column of Table 4). Compared to the rigor of psychophysics and behaviorism, Gestalt psychology was severely criticized for offering mere demonstrations, using either very simple or confounded stimuli, formulating laws with little precision, and adding new laws for every factor shown to have an influence on perceptual organization. In the 1950 s and 1960s, its critics increasingly insisted on causal explanations, by which they meant cognitive operations in the mind that could be modeled as computer algorithms or neural mechanisms that could be attributed to the properties of single cells that were discovered by Hubel and Wiesel in that period. In addition, serious conceptual limitations appeared when Gestalt thinking was extended to other areas such as personality and social psychology (e.g., Richard Crutchfield, Solomon Asch, Fritz Heider, David Krech). The further the metaphors were stretched, the harder it became to connect them to Köhler's concept of a self-organizing brain and his speculations about electromagnetic brain fields.

Despite these criticisms, Gestalt thinking did not disappear from the stage completely. In the slipstream of Shannon's information theory, a few researchers tried to provide a quantitative underpinning to the central Gestalt notion of simplicity (e.g., Attneave, 1954; Attneave \& Arnoult, 1956; Hochberg \& McAlister, 1953; Leeuwenberg, 1969, 1971; for a review, see Hatfield \& Epstein, 1985). A number of independent, original scientists working on perception and information processing kept some Gestalt issues on the research agenda (e.g., Fred Attneave, Wendell Garner, Julian Hochberg, Irvin Rock). These became more prominent again with the discovery of true Gestalt phenomena such as global precedence in hierarchical letters (e.g., Navon, 1977), configural superiority effects based on emergent features (e.g., Pomerantz, Sager, \& Stoever, 1977), and the importance of hierarchical structure in perceptual representations (e.g., Palmer, 1977). The experimental paradigms were derived from standard methods in cognitive psychology, and the results were incorporated into mainstream information-processing accounts (e.g., J. Beck, 1982; Kubovy \& Pomerantz, 1981). In the major alternative approaches to visual perception-the ecological (e.g., J. J. Gibson, 1971) and computational (e.g., Marr, 1982) approaches-the influence of Gestalt thinking has also been acknowledged explicitly. In the last 2 or 3 decades, perceptual grouping and figure-ground organizationthe most central topics of Berlin school research-have returned to center stage (e.g., Kimchi, Behrmann, \& Olson, 2003), although the relationship to the original Gestalt theory (e.g., two-sided dependency between wholes and parts, minimum principle) is not always clear.

In the remainder of this article, as well as in a second more theoretically oriented article (Wagemans et al., 2012), we review the later developments in more detail (summarized in the right

Table 4

Problems in Old-School Gestalt Psychology and How They Are Solved in Contemporary Research

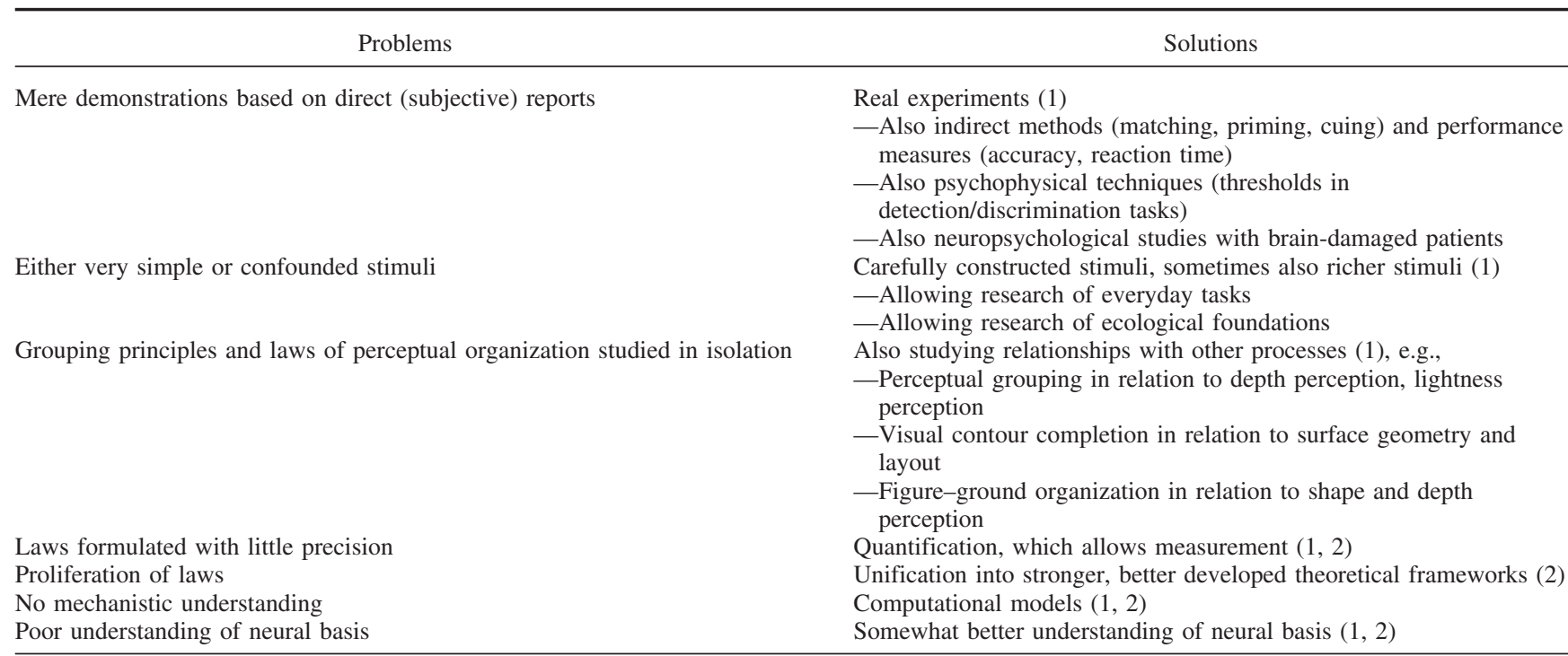

Note. $1=$ Article $1 ; 2=$ Article 2 . 
column of Table 4). We start with research on perceptual grouping in simple displays (Section 3) and extend this to contour grouping, integration, and completion in more complex shapes and realworld images (Section 4). In the next section, we cover research on figure-ground perception, where many of the factors affecting grouping, in addition to unique factors, exert an influence (Section 5). Although links to neural mechanisms are mentioned throughout, we also provide a more integrated account of the literature on the neural mechanisms of contour grouping and figure-ground organization in a separate section (Section 6). This review demonstrates that research from the last 2 or 3 decades has addressed (and partially solved) some of the major methodological and conceptual shortcomings in old-school Gestalt psychology.

\section{Perceptual Grouping}

\section{Introduction}

Historically, the visual phenomenon most closely associated with perceptual organization is grouping: the fact that observers perceive some elements of the visual field as going together more strongly than others. Indeed, perceptual grouping and perceptual organization are sometimes presented as though they were synonymous. They are not. Grouping is one particular kind of organizational phenomenon, albeit a very important one. Another is figure-ground organization. In general, grouping determines what the qualitative elements of perception are, and figure-ground determines the interpretation of those elements in terms of their shapes and relative locations in the layout of surfaces in the 3-D world.

Max Wertheimer first posed the problem of perceptual grouping in his groundbreaking 1923 paper by asking what stimulus factors influence the perceived grouping of discrete elements. He first demonstrated that equally spaced dots do not group together into larger perceptual units, except as a uniform line (see Figure 1A), and then noted that when he altered the spacing between adjacent dots so that some dots were closer than others, the closer ones grouped together strongly into pairs (see Figure 1B). This factor of relative distance, which Wertheimer called proximity, was the first of his famous laws or (more accurately) principles of grouping.

Wertheimer went on to illustrate other grouping principles, several of which are portrayed in Figure 1. Figures 1C, 1D, and 1E demonstrate different versions of the general principle of similarity: All else being equal, the most similar elements (in color, size, and orientation for these examples) tend to be grouped together. Another powerful grouping factor is common fate: All else being equal, elements that move in the same way tend to be grouped together. Notice that both common fate and proximity can actually be considered special cases of similarity grouping, with velocity and position as the relevant properties, respectively. Further factors influencing perceptual grouping of more complex elements, such as lines and curves, include symmetry (see Figure 1G), parallelism (see Figure 1H), and continuity or good continuation (see Figure 1I). Continuity is important in Figure 1I because observers perceive it as containing two continuous intersecting lines rather than as two angles whose vertices meet at a point. Figure 1J illustrates the effect of closure: All else being equal, elements that form a closed figure tend to be grouped together. This display also shows that closure can dominate continuity, since
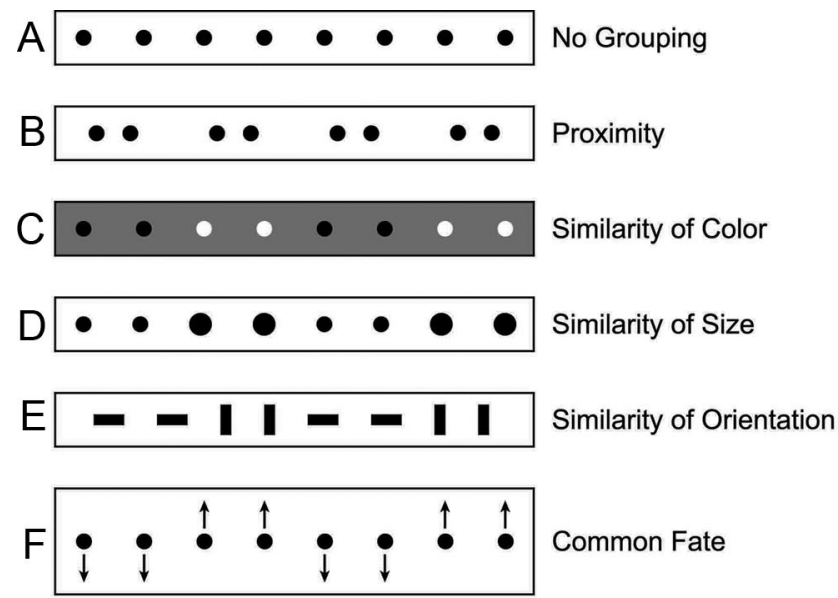

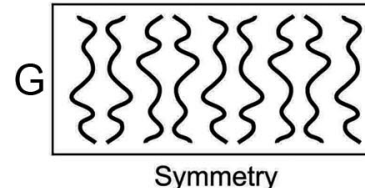

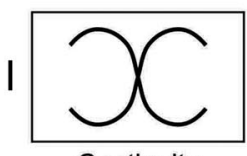

Continuity
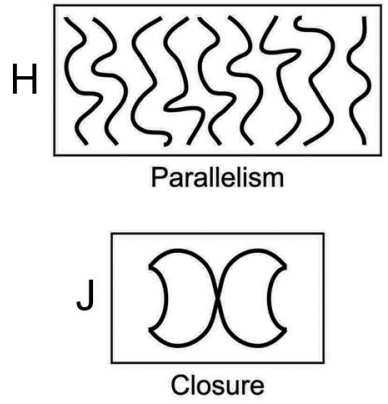

Common Region
Figure 1. Illustration of several grouping principles. Adapted from "Perceptual Organization in Vision," by S. E. Palmer, in Stevens' Handbook of Experimental Psychology: Vol. 1. Sensation and Perception (p. 183), ed. by H. Pashler, 2002, New York: NY, Wiley. Copyright 2002 by John Wiley and Sons. Adapted with permission.

the very same elements that were organized as two intersecting lines in Figure 1I are now organized as two angles meeting at a point in Figure 1J.

One might think that such grouping principles are mere textbook curiosities only distantly related to normal perception. On the contrary, they pervade virtually all perceptual experiences because they determine the objects and parts that people perceive in the environment. (Hence, they also affect other sensory modalities; for a thorough discussion of grouping principles in audition, see Bregman, 1990; for a recent review of Gestalt principles in tactile perception, see Gallace \& Spence, 2011.) A practical application of the Gestalt principles is camouflage, which results when the same grouping processes that would normally make an organism stand out from its environment as a separate object cause it to be grouped together with its surroundings instead. For instance, the same leopard that is clearly visible when it is seen in a tree against the uniform sky is difficult to see against a mottled, leafy backdrop- until it moves. Even perfect static camouflage is undone by the principle of common fate. In sum, camouflage and camouflage breaking provide an ecological rationale for the principles of grouping.

Since the early days of Gestalt psychology, considerable progress has been made, including (a) the discovery of additional 
principles, (b) the experimental measurement of the strength of grouping factors and the development of quantitative laws, as well as (c) new insights into the level of processing at which perceptual grouping happens. These new developments are described in the next section, where we also discuss their possible structural and ecological basis.

\section{New Principles of Grouping}

Generalized common fate. One of the most powerful of the classic grouping principles is common fate-the tendency for elements that move together to be perceived as a unitary entity (Wertheimer, 1923). The possibility that Wertheimer may have had a much broader range of phenomena in mind, however, is suggested by a passage of his seminal article that is not widely known because it was not included in Ellis's translation (Wertheimer, 1923/1938c): "Also this principle [of common fate] is valid in a wide range of conditions; how wide is not yet investigated here" (Wertheimer, 1923, p. 316, our own translation). In this vein, A. B. Sekuler and Bennett (2001) presented an extension of common fate to grouping by common luminance changes. They found that when elements of a visual scene become brighter or darker simultaneously, even if they have different luminances throughout, observers have a powerful tendency to group those elements perceptually. It is as though the principle of common fate operates not only for the common motion of elements through 3-D physical space, but through luminance space as well. The structural rationale for generalized common fate is clear: It is another example of similarity grouping, but based on similarity of changes in feature values, such as luminance or position, rather than on the similarity of the feature values themselves. An ecological rationale for grouping by common luminance changes might lie in the simultaneous brightening or darkening that occurs across a spatial area when the level of illumination changes (e.g., with the appearance of sunlight or shadows; see also van den Berg, Kubovy, \& Schirillo, 2011).

Synchrony. Synchrony is the tendency for elements that change simultaneously to be grouped together (Alais, Blake, \& Lee, 1998; S.-H. Lee \& Blake, 1999). The changes do not have to be in the same direction, however, as they do in generalized common fate. A random field of black and white dots whose luminances change in polarity randomly over time against a gray background, for example, will segregate into two distinct regions if the dots in one area change synchronously rather than randomly. Grouping by synchrony can be considered as an even more general form of common fate in which the simultaneous changes do not have to involve either motion, as in classic common fate, or common direction of change, as in generalized common fate. The structural basis for grouping by synchrony is clear: the simultaneous occurrence of visible changes of the elements that are grouped. Such grouping makes sense because it reflects a strong temporal regularity in the stimulus event.

The ecological rationale behind grouping by synchrony is far less clear, however. Objects in the natural environment seldom change their properties in different directions or along different dimensions in temporal synchrony. Indeed, it is difficult even to devise plausible examples of ecological situations that would exhibit this kind of temporal regularity without some form of extended common fate being involved. Nevertheless, synchrony grouping may arise from some very general nonaccidentalness detection mechanism, possibly connected to the perception of causality (e.g., Michotte, 1946/1963). The argument is that the temporal coincidence of multiple changes is unlikely to be due to chance alone, and so it must have some common underlying cause related to an ecological event that relates the synchronously changing elements.

A radically different and quite controversial rationale is that temporal synchrony of changes drives grouping because synchrony of neural firings is the physiological mechanism by which the brain codes all forms of grouping (e.g., Milner, 1974; von der Malsburg, 1981). The argument is that if the environment drives the neural substrate to produce synchronous firing by virtue of synchronous changes, the changing elements will automatically be grouped because of the synchronous firing. Some researchers report evidence that seems to support this claim (e.g., Gray \& Singer, 1989; Singer \& Gray, 1995), but others disagree (e.g., Shadlen \& Movshon, 1999). This issue is discussed further in the second article (Wagemans et al., 2012, Section 4). Further controversy surrounds synchrony grouping because it has been claimed that such grouping effects are actually produced by stimulus artifacts that can be detected by the early visual system (Farid, 2002; Farid \& Adelson, 2001). These challenges are complex, but the bottom line is that both the existence of grouping synchrony and the mechanism by which it occurs are currently unclear. In general, these controversies show quite clearly that the interest in perceptual grouping principles remains strong in contemporary research.

Common region. Common region is the tendency for elements that lie within the same bounded area (or region) to be grouped together (Palmer, 1992). An illustration is provided in Figure $1 \mathrm{~K}$, where the black dots that lie within the same ovals are likely to be grouped into pairs. The structural basis for grouping by common region appears to be that all the elements within a given region share the topological property of being inside of or contained by some larger surrounding contour. If it is viewed as similarity of containment, it can be related to several other grouping principles based on similarity (e.g., color, orientation, and size). Common region also appears to have an ecological rationale arising from textures and hierarchically embedded parts. When a bounded region encloses a number of image elements, they are likely to be elements on the surface of a single object, such as a leopard's spots or the features of a face, rather than independent objects that just happen accidentally to lie within the same bounding contour.

Experimental evidence for the existence of common region as a grouping factor comes from studies using the repetition discrimination time or RDT method (D. M. Beck \& Palmer, 2002; Palmer $\&$ Beck, 2007). In a speeded discrimination task, observers were able to report the shape of a repeated element more quickly in a line of otherwise alternating shapes (e.g., squares and circles) when the repeated shapes were located within the same surrounding region than when they were located in two separate regions.

Element connectedness. Element connectedness is the tendency for distinct elements that share a common border to be grouped together. The important structural basis for this form of grouping is the topological property of connectedness (Palmer \& Rock, 1994). Connectedness can be considered as the limiting case of the classic factor of proximity, but Palmer and Rock (1994) argued that framing it this way puts the cart before the horse, in the 
sense that one needs distinct units to speak meaningfully about their distance in the first place. The compelling rationale for element connectedness is ecological: Pieces of matter that are physically connected to each other in 3-D space are the primary candidates for being parts of the same object, largely because they tend to behave as a single unit. The bristles, metal band, and handle of a paint brush, for example, constitute a single object in large part because of their connectedness, as demonstrated by the fact that when you push one part, the other parts move rigidly along with it.

The effectiveness of element connectedness was demonstrated in a behavioral task using the RDT method (Palmer \& Beck, 2007). As was the case for grouping by common region, displays with elements that were connected to each other produced reliably faster responses than displays with unconnected elements. Another behavioral result that provides striking support for the importance of element connectedness comes from a neuropsychological study by Humphreys and Riddoch (1993). Their patient, who was afflicted with Balint's syndrome-a condition resulting from bilateral damage to parietal cortex that results in a deficit in perceiving more than a single object at any given time-was unable to discriminate between arrays containing many circles of just one color (either all red or all green) and arrays in which half of the circles were red and the other half green. However, if pairs consisting of one red circle and one green circle were connected by lines, the same patient was able to make the discrimination between one-color and two-color arrays. Unifying a pair of circles through element connectedness thus appears to enable these patients to perceive them as a single perceptual object so that they could see two circles at once, a feat that was impossible for them in the absence of the connecting line.

Uniform connectedness. This principle represents something of a departure from standard Gestalt ideas about perceptual organization because it addresses the question of how the initial organization into elements might occur. In his classic article on grouping, Wertheimer (1923) never actually mentioned where the to-be-grouped elements came from. Presumably, he believed that they were somehow derived from the grouping principles he articulated, but Palmer and Rock (1994) argued that they arise from the earlier organizational process of uniform connectedness (UC), which is the principle by which the visual system initially partitions an image into a set of mutually exclusive connected regions having uniform (or smoothly changing) properties, such as luminance, color, texture, motion, and depth. The UC elements thus created form the entry level units into a part-whole hierarchy that is created by grouping together different UC regions and, if necessary, by parsing them into lower level elements at deep concavities (e.g., Hoffman \& Richards, 1984).

Palmer and Rock's (1994) claims regarding the foundational status of UC have not been uniformly accepted. Peterson (1994), for instance, argued that UC is one of many properties relevant to partitioning the visual field, and that UC units are not entry-level units. Kimchi (2000) examined the role of UC in experiments designed to reveal the gradual emergence, or microgenesis, of organizational processes using a primed matching task with displays containing connected or disconnected elements. The complex results she obtained were not consistent with UC being the sole determinant of entry-level units in a part-whole hierarchy, as Palmer and Rock proposed. Rather, they showed that collinearity and closure were at least as important, if not more so, in the initial organization that can be tapped by such methods. Nevertheless, the theoretical rationale for some organizational process like UC to contribute to creating a set of potential perceptual units on which further grouping and parsing can operate seems sound. Indeed, something like it is a standard assumption in most theories of computational vision (e.g., Marr, 1982).

Whereas the research on grouping principles, as described above, provides predictions about what elements in a display are likely to be grouped together, it does not reveal how strong each of the grouping principles is. This is the focus of the next sections, covering studies applying static and dynamic stimuli, respectively.

\section{Grouping Principles in Discrete Static Patterns}

Conceptual background. Starting with Wertheimer (1923), researchers have often used dot lattices to quantify grouping. A dot lattice is a collection of dots in the plane that is invariant under at least two translations, $a$ (with length $|a|$ ) and $b$ (whose length is $|b| \geq|a|$ ). These two lengths, and the angle between the vectors, $\gamma$ (constrained by $60^{\circ} \leq \gamma \leq 90^{\circ}$ ), define the basic structure of the lattice by defining the parallelogram between each quartet of dots in the lattice (Kubovy, 1994, extending the work of Bravais, 1850/1949; see Figure 2A). The diagonals of this parallelogram are denoted $c$ and $d$ (where $|c| \leq|d|$ ). In its canonical orientation, $a$ is horizontal.
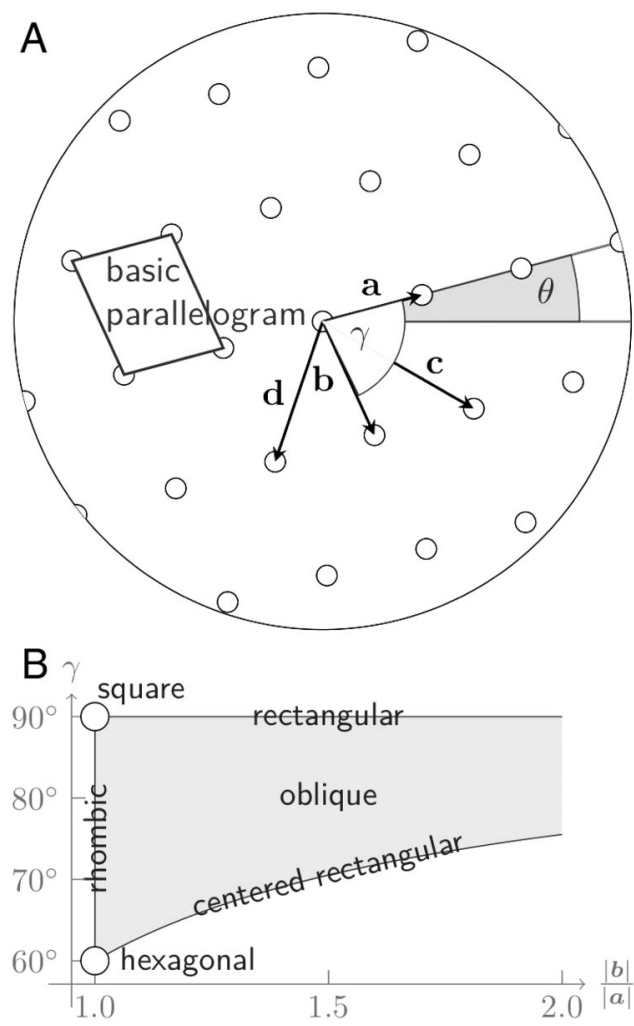

Figure 2. A: Defining features of a dot lattice stimulus. B: Twodimensional space and nomenclature of dot lattices. Adapted from "The Perceptual Organization of Dot Lattices," by M. Kubovy, 1994, Psychonomic Bulletin \& Review, 1, pp. 183, 184, 187. Copyright 1994 by Springer. Adapted with permission. 
More generally, the orientation of the lattice can be defined by the angle $\theta$ (measured counterclockwise) and $|a|$ is called the scale of the lattice. Since scale is irrelevant to the invariant properties of the lattice and unimportant for grouping over a reasonable range, the relevant parameters are $|b| /|a|$ and $\gamma$ (see Figure 2B). With these two parameters, six different types of lattices can be defined, each characterized by their symmetry properties.

When grouping by proximity is pitted against grouping by similarity, displays consist of at least two kinds of elements (called motifs), separated by one of the translation components, resulting in dimotif lattices (see Figure 3; Grünbaum \& Shephard, 1987). In order to characterize the relation between two grouping principles, one must construct grouping indifference curves (see Figure 4), similar to the indifference curves used in microeconomics (Krantz, Luce, Suppes, \& Tversky, 1971): Imagine a consumer who would be equally satisfied with a market basket consisting of $1 \mathrm{~kg}$ of meat and $4 \mathrm{~kg}$ of potatoes and another consisting of $2 \mathrm{~kg}$ of meat and 1
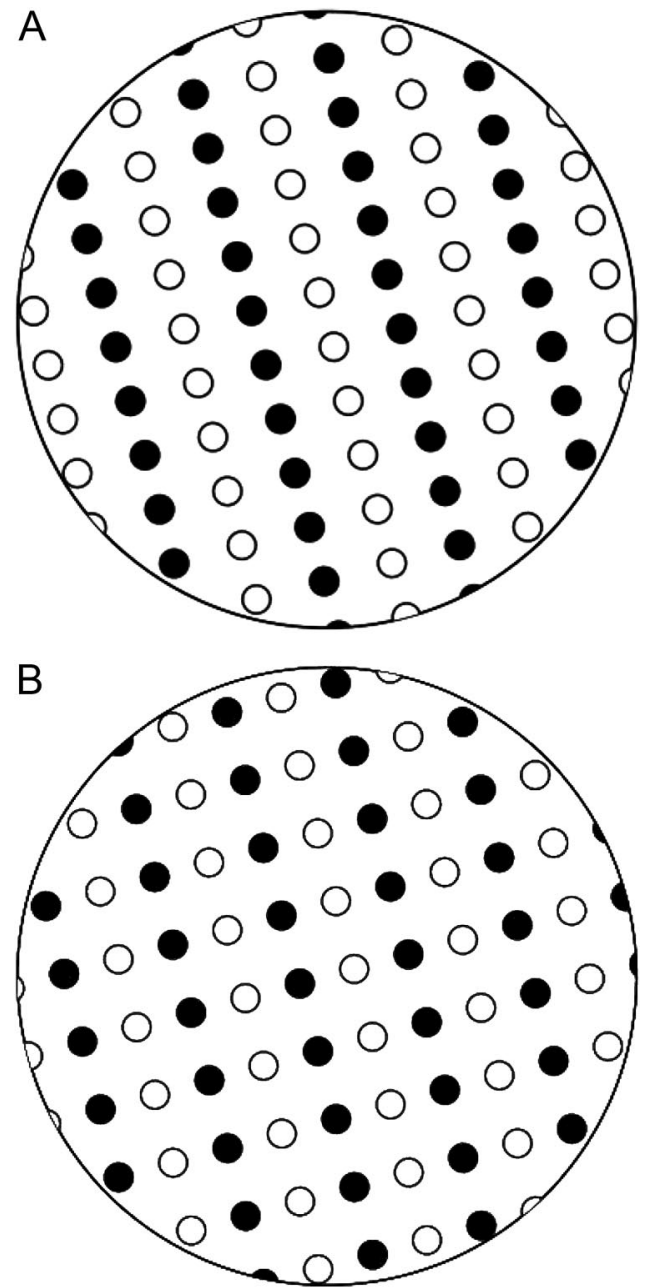

Figure 3. Two dimotif rectangular dot lattices with $|b| /|a|=1.2$. A: The elements are the same in the direction of the shortest distance and different along the second shortest distance. Grouping by proximity is reinforced by similarity. B: The elements are different in the direction of the shortest distance and same along the second shortest distance. Grouping by proximity is opposed by similarity. $\mathrm{kg}$ of potatoes. In such a case, the (meat, potato) pairs $(1,4)$ and $(2,1)$ both lie on an indifference curve.

With these tools in hand, two important questions regarding perceptual grouping can be formulated. First, when several orientations can be perceived based on grouping by proximity in a particular dot lattice, what determines the preferred grouping? Is the outcome determined by the relative distance alone, or also by the angle between the competing organizations (an aspect that affects the global symmetry of the lattice and the way the overall configuration looks)? Second, when grouping by proximity and grouping by similarity are concurrently applied to the same pattern, what rule governs their joint application? Are these two principles combined additively or not? We briefly review the most important attempts to address these two questions. The initia studies always pitted grouping by proximity against grouping by similarity.

Initial attempts to quantify grouping by proximity by pitting it against similarity. The first to systematically study grouping by proximity in interaction with similarity was Rush (1937). In her experiment, she showed observers sequences of dot lattices in which the distance between dots in one orientation was held constant, and the distance between dots in another orientation was reduced from trial to trial. She assumed-incorrectly, as argued below-that one could measure the strength of the two principles by finding their point of equilibrium, and concluded that "it may be said that Similarity equals about $1.5 \mathrm{~cm}$ of Proximity" (Rush, 1937, p. 90).

Roughly 2 decades later, Hochberg and Silverstein (1956, unaware of Rush's work, as a footnote in Hochberg \& Hardy, 1960, attests) also set out to solve the problem of measuring the strength of grouping by similarity by pitting it against grouping by proximity. In manipulating luminance differences or distances between dots, they produced grouping indifference curves (see Figure 4). Reanalysis showed that an additive combination of proximity and similarity described their results best. Unfortunately, the logic employed by Hochberg and his colleagues suffered from the same flaw as Rush. Their method produced only one grouping indifference curve- the one for which both groupings are in equilibrium. Their method cannot produce grouping indifference curves for which one principle is 2 times or 3 times as strong as the other, which are needed to measure the relative strengths of the two principles.

Quinlan and Wilton (1998) studied the relations between grouping by proximity and two forms of grouping by similarity (by color and by shape). Their stimuli consisted of strips of seven elements, with the center element as the target. They manipulated proximity by slightly shifting the left or right set of three elements, and they also manipulated color and shape similarity. Observers were asked to rate the degree to which the target grouped with the elements on the left or on the right. Although the conception of the experiment is elegant, its reach was curtailed because each grouping principle was either present or absent. Had Quinlan and Wilton used a design in which each type of grouping was a multilevel factor, they could have addressed the additivity question (i.e., the second question introduced above), but they did not.

Oyama, Simizu, and Tozawa (1999) presented rectangular dimotif lattices for $3 \mathrm{~s}$ and asked observers to indicate continuously with a joystick whether they saw horizontal or vertical grouping. The horizontal separation was increased by $15^{\prime}$ after a 


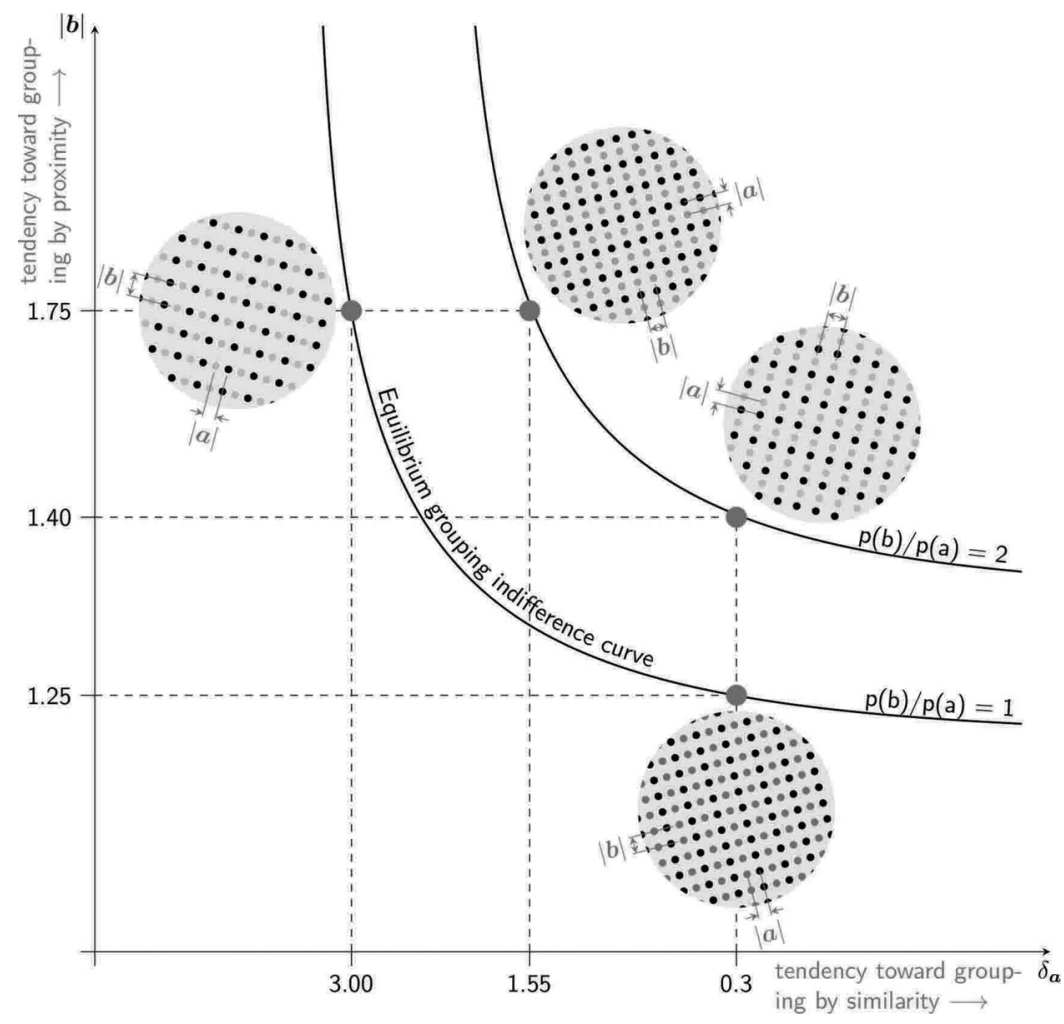

Figure 4. Two grouping indifference curves. The abscissa, $\delta_{a}$, represents the difference in luminance between adjacent elements of $a$. The ordinate, $|b|$, represents the distance between the dots of $b$ (assuming $|a|=1$ ). Only the equilibrium grouping indifference curve is achievable without independently measuring the strength of grouping by proximity. The methods to be described later allow us to plot a family of indifference curves. (The $\theta$ values are different for each of the four dot lattices.)

horizontal response, and decreased by that amount after a vertical response. Using a double-staircase method, the ratio of vertical to horizontal distances was determined that matched a particular dissimilarity. This method produced more complete equilibrium grouping indifference curves than were obtained by Hochberg and his colleagues.

The pure distance law and the additivity of grouping principles. Oyama (1961) was the first to show that one can measure the strength of grouping by proximity without pitting it against another grouping principle (e.g., grouping by similarity). Using rectangular dot lattices at a fixed orientation, he recorded the amount of time subjects reported seeing the competing horizontal and vertical groupings. The ratio of the time they saw the horizontal and vertical organizations was found to be a power function of the ratio of the horizontal and vertical distances $t_{\mathrm{h}} / t_{\mathrm{v}}=\left(d_{\mathrm{h}} /\right.$ $\left.d_{\mathrm{v}}\right)^{-\alpha}$, with $\alpha \approx 2.89$.

Using dot lattices at near equilibrium, Kubovy and Wagemans (1995) and Kubovy, Holcombe, and Wagemans (1998) demonstrated that grouping by proximity can be understood as the outcome of a probabilistic competition among potential perceptual organizations. The basic idea is simple. If the distances between dots in two orientations of the lattice are equal, the chances of seeing one orientation or the other are equal too. If one distance becomes larger than the other, the relative chance of seeing that orientation decreases. If the ratio of the longer to the shorter vector is larger than about 1.5 , grouping along that orientation is almost never seen. Kubovy and colleagues presented different kinds of dot lattices for $300 \mathrm{~ms}$ each and asked observers to indicate the perceived orientation. They could then use the frequencies of the perceived orientations over a large number of trials as estimates of the probabilities, and plot the relative frequencies as a function of relative distance. Their results (shown schematically in Figure 5) were remarkable. All the values of the log-odds fell on the same line, called the attraction function. Its slope is a person-dependent measure of sensitivity to proximity. Although the (relative) strength of grouping decays as an exponential function of (relative) distance, the attraction function in log-space is linear. The fact that all data points - obtained with all pairs of distances and all relative orientations (i.e., all points in the 2-D lattice space of Figure 2B) — could be fitted well by a single straight line indicates that grouping by proximity depends only on the relative distance between dots in competing organizations, not on the overall configuration in which the competition occurs (i.e., the lattice type, each with its own symmetry properties). Hence, this result, which was called the pure distance law, provided a satisfactory answer to the first question raised above.

Once it has been established how grouping varies as a function of relative distance, the effect of conjoined grouping principles can be determined by measuring a family of grouping indifference curves. Kubovy and van den Berg (2008) presented participants 


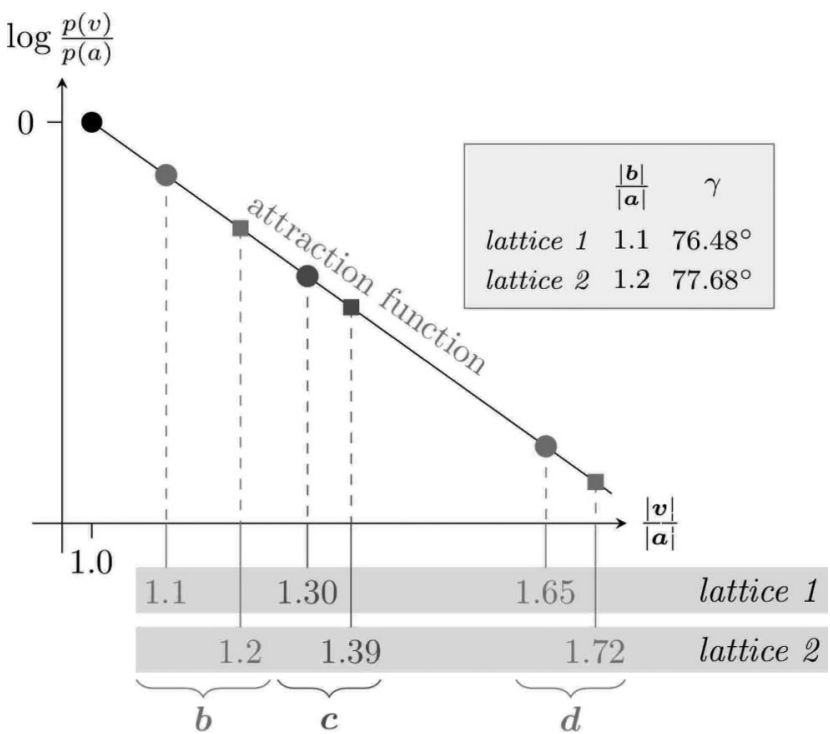

Figure 5. The pure distance law. Adapted from "On the Lawfulness of Grouping by Proximity," by M. Kubovy, A. O. Holcombe, and J. Wagemans, 1998, Cognitive Psychology, 35, p. 89. Copyright 1998 by Elsevier. Adapted with permission.

with rectangular lattices of dots of different contrasts. Dots with the same contrast were either arranged along the shorter axis of each rectangle of dots within the lattice (similarity and proximity in concert) or arranged along the longer axis (similarity and proximity working against each other). Dot lattices varied across two dimensions: the ratio between the short and long axes of each rectangle of dots within the lattice and the contrast difference between the different arrays of dots. As in the previous studies, each lattice was presented for $300 \mathrm{~ms}$, and participants were asked to indicate which of the four orientations best matched the perceived arrangement of the dots in the lattice. By plotting the log likelihood of reporting the direction of the long axis versus the short axis as a function of the ratio of the length of the long and short axis for different values of the contrast difference between dots (shown schematically in Figure 6A), a family of grouping indifference curves was then obtained (depicted in Figure 6B). Because these indifference curves are parallel in log-odds space, the conjoined effects of proximity and similarity are called additive. Using lattices in which dots were replaced by Gabor elements, Claessens and Wagemans (2005) came to similar conclusions regarding proximity and collinearity. These results, therefore, provide a clear answer to the second question raised above.

\section{Grouping Principles in Discrete Dynamic Patterns}

Apparent motion is perceived when an object is presented in two or more successive frames at different spatial locations with proper durations and intervals. As discussed before, Wertheimer (1912) showed that under certain conditions it is possible to perceive pure motion, where motion is perceived without perceiving the moving object itself. The optimal timing and spacing between successively presented object presentations were investigated in more detail by Korte (1915), who found a direct relationship between the optimal temporal and the optimal spatial intervals for perceived apparent motion. Later studies, however, have shown that the relationship between the optimal temporal and spatial intervals depends on the stimuli used. For example, using horizontal arrays of dots that were displaced on successive frames, Burt and Sperling (1981)
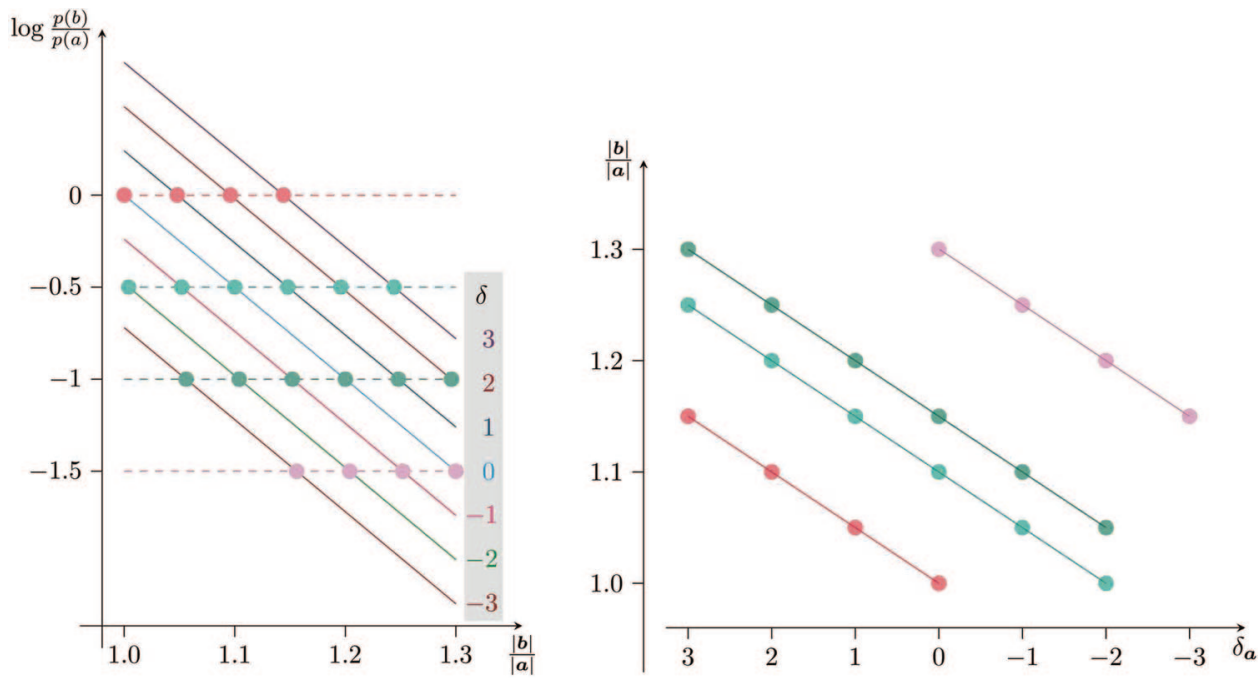

(A)

(B)

Figure 6. The conjoined effects of proximity and similarity are additive. The dashed lines in Panel A turn into grouping indifference curves in Panel B. Adapted from "The Whole Is Equal to the Sum of Its Parts: A Probabilistic Model of Grouping by Proximity and Similarity in Regular Patterns," by M. Kubovy and M. van den Berg, 2008, Psychological Review, 115, pp. 147-150. Copyright 2008 by the American Psychological Association. 
found that the spacing of the dots in the array strongly influenced the apparent motion percept in addition to the effects of the temporal interval between frames and the spatial displacement of the entire array.

The influence of spatial and temporal factors in apparent motion was further investigated using the Ternus display (Kramer \& Yantis, 1997; Pantle \& Picciano, 1976; Ternus, 1926; Wallace \& Scott-Samuel, 2007), in which an array of three dots is presented across two frames at different spatial locations. When the two frames are presented in rapid succession (i.e., with a short interstimulus interval), it appears that the outmost dot is displaced while the center two dots appear to be stationary: Element motion occurs. When the temporal interval between the successive frames is longer, the entire array of dots appears to jump: Group motion is perceived. The two different types of perceived apparent motion represent two different solutions to the correspondence problem (Ullman, 1979), referring to the task of matching the objects in the first frame to the (possibly displaced) objects in the second frame. Whether element or group motion was perceived was found to depend on the properties of the individual stimuli in both frames such as their features (Dawson, Nevin-Meadows, \& Wright, 1994), their size or the sharpness of their edges (Casco, 1990), as well as on the presence of contextual elements affecting how they are grouped (Kramer \& Yantis, 1997) or how they are perceived in 3-D space (He \& Ooi, 1999).

The interaction between spatial and temporal aspects was further investigated by Gepshtein and Kubovy (2000), who were able to determine the relationship between spatial grouping (determining which elements in each frame belong together) and temporal grouping (determining which elements across frames belong together), by using successive presentations of dot lattices-motion lattices-which allowed them to independently manipulate the strength of spatial and temporal groupings. A motion lattice (see Figure 7) is composed of two identical dot lattices, $D_{1}$ and $D_{2}$, displayed in alternation. Two ratios determine the perceived motion: (a) the motion ratio $r_{m}=m_{2} / m_{1}$, where $m_{1}$ and $m_{2}$ are the shortest and the next shortest spatial distances across which the

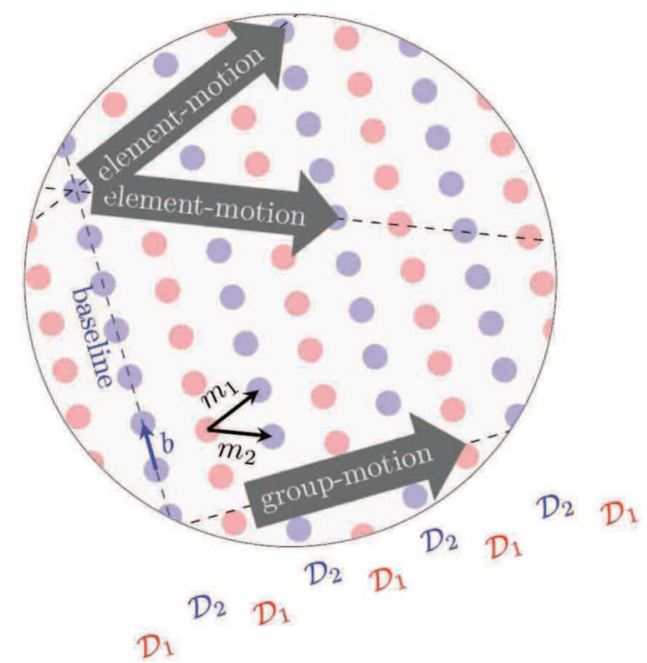

Figure 7. A motion lattice. $D_{1}$ and $D_{2}$ are dot lattices presented in alternation. apparent motion could occur between the frames; (b) the baseline ratio $r_{b}=b / m_{1}$, where $b$ is the shortest spatial distance between the dots within $D_{1}$ and $D_{2}$ to which the apparent motion could apply. The orientation of a virtual line drawn through these dots is called the baseline orientation.

As in the classic Ternus display, two classes of motion can be perceived. First, element motion is now apparent motion from each dot in $D_{1}$ to a corresponding dot in $D_{2}$ (and vice versa as the dot lattices alternate). The log-odds of seeing $m_{2}$ rather than $m_{1}$ as a function of the ratio of the distances is called an affinity function, by analogy with the concept of an attraction function for static dot lattices (see Figure 8A). Second, group motion is now apparent motion orthogonal to the baseline orientation (see Figure 7). Sequential models predict that if the spatial configuration of a stimulus remains constant, the likelihood of seeing group motion-an indicator of spatial grouping-cannot be affected by manipulations of the temporal configuration of the stimulus. However, the pattern of interaction in Figure 8B between $r_{m}$, the temporal configuration of the stimulus, and $r_{b}$, the relative density of the dots along the baseline, clearly refutes the sequential model.

How spatial and temporal distances interact to determine the strength of apparent motion has been controversial. Some studies report space-time coupling: If the spatial or temporal distance between successive stimuli is increased, the other distance between them must also be increased to maintain a constant strength of apparent motion (i.e., Korte's third law of motion). Other studies report space-time tradeoff: If one of the distances is increased, the other must be decreased to maintain a constant strength of apparent motion. To establish what determines whether coupling or tradeoff occurs, Gepshtein and Kubovy (2007) generalized the motion lattice of Figure 7, as illustrated in Figure 9, showing a temporal component of $m_{3}, T_{3}$, of twice the magnitude of the temporal component of $m_{1}, T_{1}$. By manipulating the spatial components of these motions, $S_{3}$ and $S_{1}$ from $S_{3} \gg S_{1}$ to $S_{3} \ll S_{1}$, an equilibrium point between the extremes was found at $r_{31}=S_{3} / S_{1}$, for which the probability of seeing the two motions was the same. If $r_{31}>1$, then space-time coupling holds; if $r_{31}<1$, then space-time tradeoff holds. This suggests that previous findings on apparent motion were special cases and that the allegedly inconsistent results can be embraced by a simple law in which a smooth transition from tradeoff to coupling occurs as a function of speed: Tradeoff holds at low speeds of motion (below $\approx 12 \%$ ), whereas coupling (Korte's law) holds at high speeds. The deeper theoretical implications of these results for the visual system's economy principles are discussed in the second review article (Wagemans et al., 2012, Section 4).

The above research on perceptual grouping in static and dynamic discrete patterns spans a complete century, from Schumann (1900), Wertheimer (1912, 1923), and Korte (1915) up until today. It mainly used well-controlled, parametrically varied stimuli in order to isolate one factor or another, and trying to quantify its strength. In addition, it sparked a renewed interest in understanding the level at which perceptual grouping operates, which was addressed in studies that used somewhat richer stimuli with additional variations, more typical for naturally occurring stimulation. 
A

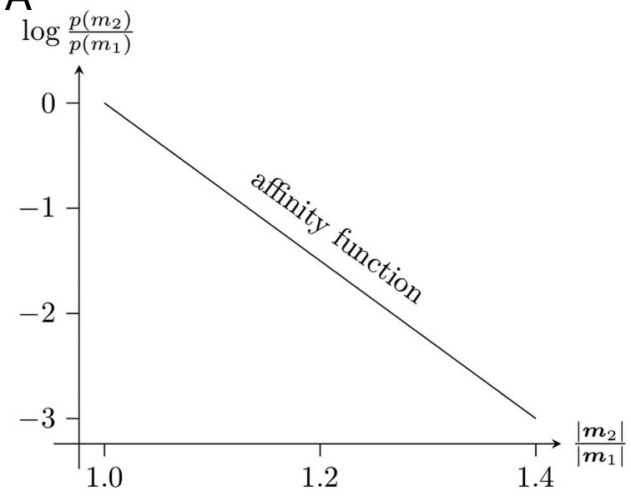

B

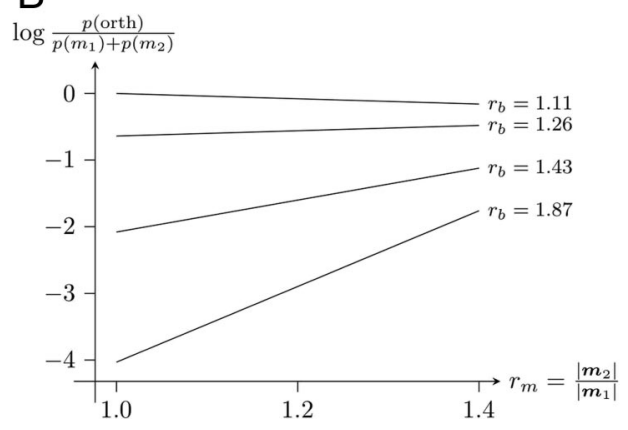

Figure 8. A: The affinity function. B: The objecthood functions.

\section{At What Level Does Grouping Happen?}

As described above, Wertheimer (1923) demonstrated powerful grouping effects due to a large number of stimulus variables (e.g., proximity, similarity, good continuation) using flat 2-D displays on the printed page (see Figure 1). Subsequent researchers have investigated where in the visual system these effects occur (i.e., before or after the construction of a 3-D representation of the image), by using various kinds of 3-D displays with depth cues, shadows, transparency, and other higher level factors.

Rock and Brosgole (1964) conducted a classic experiment on this topic to examine whether grouping by proximity operated on retinal 2-D distances or perceived 3-D distances. Observers in a dark room saw a 2-D array of luminous beads either in the frontal plane (perpendicular to the line of sight) or slanted in depth so that the horizontal dimension of the array was foreshortened. The beads were actually closer together vertically than horizontally, so that when they were viewed in the frontal plane, observers always reported seeing them grouped into vertical columns rather than horizontal rows. The critical question was whether or not the beads would be grouped in the same way when the same lattice was viewed slanted in depth such that the beads were retinally closer together in the horizontal direction. When this array was viewed monocularly, so that the beads appeared to be in a frontal plane perpendicular to the line of sight (even though they were actually slanted in depth), observers perceived the grouping to change to a set of rows rather than columns, as one would expect based on retinal distances. However, when viewed binocularly, so that stereoscopic depth information enabled observers to see the beads slanted in depth, they reported grouping them into vertical columns, as predicted by postconstancy grouping based on a 3-D representation of perceived distances in the phenomenal environment (because the beads appeared to be closer in the vertical direction, as was actually the case in the physical world). Rock and Brosgole's results therefore support the hypothesis that the final, conscious result of grouping occurs after binocular depth percep-
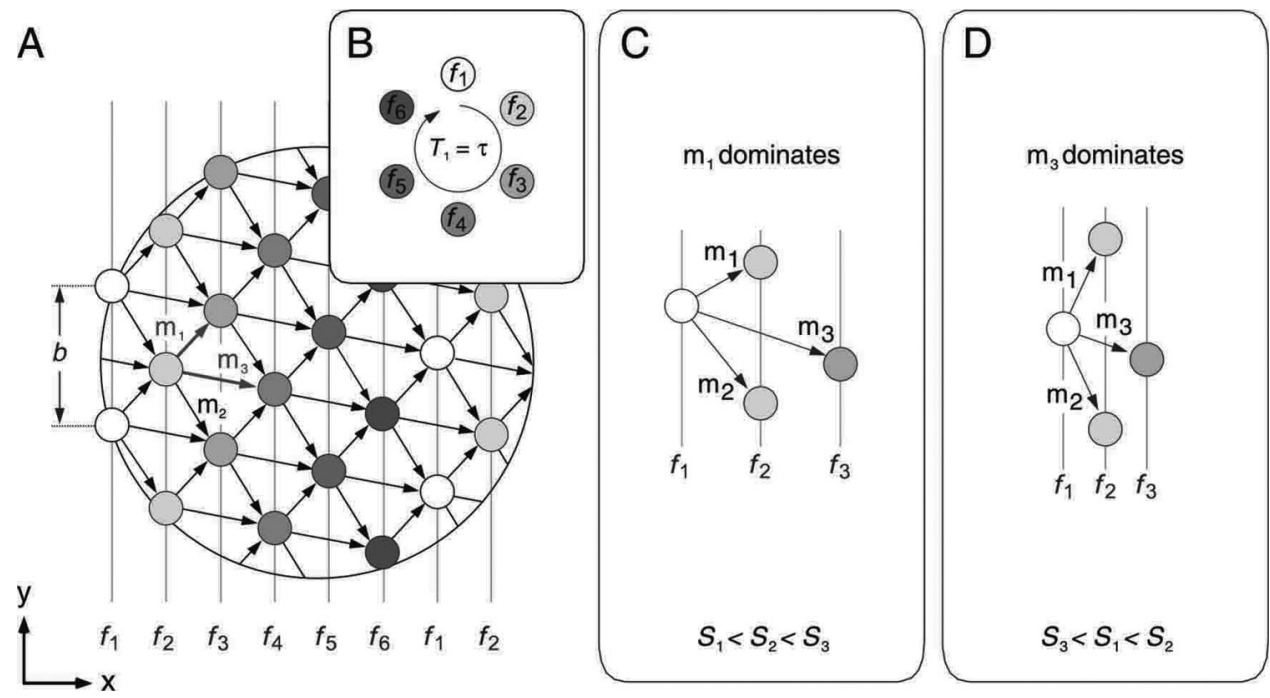

Figure 9. A six-stroke motion lattice. A: The successive frames are superimposed in space. Gray levels indicate time. $b$ is the baseline distance. B: The time course of the display. The three most likely motions along $m_{1}, m_{2}$, and $m_{3}$ can occur because dots in frame $f_{i}$ can match dots in either frame $f_{i+1}$ or frame $f_{i+2}$. C-D: Conditions in which different motion paths dominate: $m_{1}$ in Panel C and $m_{3}$ in Panel D. (The stimuli were designed so that $m_{2}$ would never dominate.) Adapted from "The Lawful Perception of Apparent Motion," by S. Gepshtein and M. Kubovy, 2007, Journal of Vision, 7(8), Article 9, p. 7. Copyright 2007 by the Association for Research in Vision and Ophthalmology. Adapted with permission. 
tion. Several phenomenological demonstrations supporting the same conclusion were provided by Palmer (2002a; Palmer, Brooks \& Nelson, 2003).

Rock, Nijhawan, Palmer, and Tudor (1992) later investigated whether grouping based on lightness similarity happened before or after lightness constancy. Using displays that employed cast shadows and translucent overlays, they also found evidence that the final conscious result of grouping depended on a postconstancy representation that reflected the perceived reflectance of surfaces rather than the luminance of retinal regions. Analogous evidence that the final conscious organization resulted from a grouping process that operates on relatively late, postconstancy representations was reported by Palmer, Neff, and Beck (1996) for amodal completion and by Palmer and Nelson (2000) for illusory contours. Further results of Schulz and Sanocki (2003) support the view that prior to achieving the conscious result of perceptual grouping based on a 3-D postconstancy representation, some nonconscious grouping processes operate on a 2-D preconstancy representation. They used the same lightness displays as did Rock et al., but included a brief, masked presentation condition in which they found that observers reported seeing an organization based on the retinal luminance of 2-D regions (see also van den Berg et al., 2011). Further evidence that grouping operations occur before constancy has been achieved is based on grouping effects that actually influence the achievement of constancy (see Palmer, 2003).

Perhaps the most parsimonious view consistent with the known facts is that grouping principles operate at multiple levels. It seems most likely that provisional grouping takes place at each stage of processing, possibly with feedback from higher levels to lower ones, until a final, conscious experience arises of a grouping that is consistent with the perceived structure of the 3-D environment. Whereas the above findings provide valuable information about the stages at which grouping operates, these studies have mainly employed relatively artificial stimuli. The next section is dedicated to the role of grouping in contour integration and completion, in ways that are closer to the processing of natural stimuli.

\section{Contour Integration and Completion}

\section{Introduction}

Studies in which grouping factors are isolated to quantify their strength are useful but understanding their role in everyday perception requires a different approach. An important task of natural vision is to identify and group together the portions of the 2-D retinal image that project from an object. In the simple case in which the object boundary projects as a single closed curve, the problem reduces to a problem of contour grouping or contour integration. From the 50-year history of computer vision research, however, we know that this is a computationally difficult problem for a number of reasons (e.g., Elder, Krupnik, \& Johnston, 2003). First, occlusions occur generically in natural images, resulting in a projection of the rim of the object as a disconnected set of contour fragments (see Figure 10). Also, where the figure-ground contrast is low, portions of the contour may not be detected, resulting in further fragmentation. To complicate matters further, natural images are often highly cluttered, such that for any given contour fragment, multiple other fragments could be the correct continua-

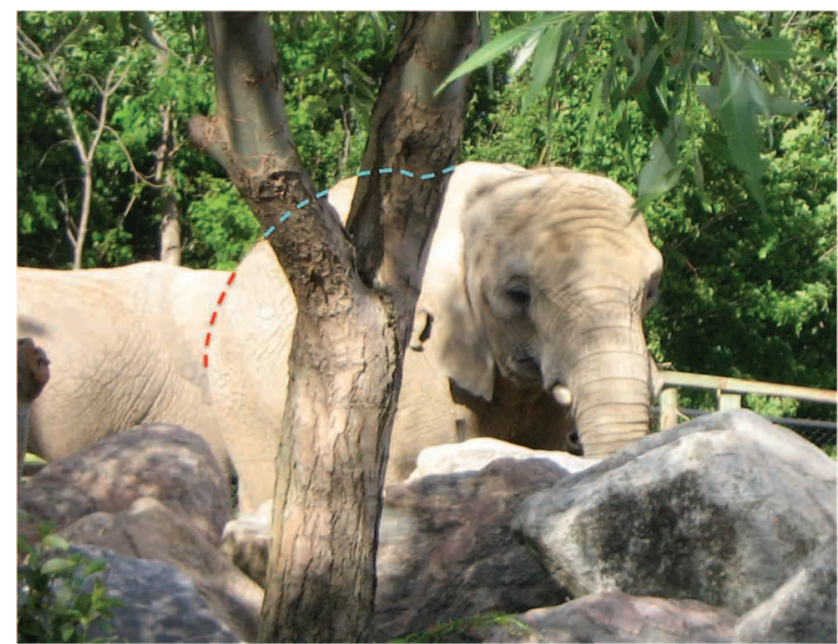

Figure 10. Object boundaries project to the image as fragmented contours, due to occlusions (dashed cyan line) and low figure/ground contrast (dashed red line).

tion of the contour. Thus, to effectively exploit contours for object segmentation, the visual system must be able to cope with uncertainty, using a relaxed form of perceptual contour closure that can work reliably even for fragmented contours (e.g., Elder \& Zucker, 1993). Discovering the nature of these mechanisms is a central topic in modern perceptual organization research, involving a range of different methodologies, including psychophysics, neurophysiology, neuroimaging, ecological statistics, and computational modeling.

Addressing the role of grouping under natural conditions requires a definition of the grouping primitives: What exactly are the elements being grouped? In the research with dot lattices reviewed above, the primitives were zero-dimensional points or dots, and proximity and similarity were the only cues involved. The study of contour grouping over oriented primitives such as bars or edges expands the grouping cues to also include good continuation. This maps directly onto related computer vision research, where the contour grouping problem is typically defined over local oriented edge elements detected using oriented linear filters (e.g., Canny, 1983) that can be loosely identified with receptive fields of simple cells in primate V1 (Hubel \& Wiesel, 1968). Even higher level primitives can be used, for instance, connected contour fragments of arbitrary shape, which may have been separated by occlusions. This higher level of representation may play a role in perceptual completion (see also below), and could be mapped to extrastriate visual areas such as V4, where neurons are known to be selective for higher order properties of shape (Pasupathy \& Connor, 1999). In psychophysics, this has motivated the use of fragmented object pictures, which can be used to study the dynamic interplay between perceptual grouping and object identification (e.g., Panis \& Wagemans, 2009).

In the following subsections, we first review the research on the role of grouping principles for contour integration-research that generalizes properties discovered for individual cues in isolation to more natural conditions where multiple cues are present and the visual stimulus may be quite complex. In addition to psychophys- 
ical results, we review the ecological foundations of the problem, and discuss computational principles and possible neural mechanisms. We then turn to the specific problem of contour completion in cases of occlusion, before discussing general issues pertaining to both perceptual grouping and contour integration.

\section{Grouping Principles for Contour Integration}

Proximity. The principle of proximity states that the strength of grouping between two elements increases as these elements are brought nearer to each other, but how exactly does grouping strength vary as a function of their separation? As reviewed above, Oyama (1961) found that this relationship could be accurately described as a power law, whereas Kubovy and Wagemans (1995) employed an exponential model, consistent with random-walk models of contour formation (Mumford, 1992; Williams \& Jacobs, 1997). However, Kubovy et al. (1998) also noted that a power law model could fit their data equally well and found that the proximity cue was approximately scale invariant: Scaling all distances by the same factor did not affect results. Since the power law is the only perfectly scale-invariant distribution, this last result adds strength to the power law model of proximity, which has been used in subsequent studies (e.g., Claessens \& Wagemans, 2008).

Perceptual scale invariance is rational if in fact the proximity of elements along real contours in natural images is scale invariantthat is, if the ecological distribution follows a power law. In support of this idea, Sigman, Cecchi, Gilbert, and Magnasco (2001) reported that the spatial correlation in the response of collinearly oriented filters to natural images does indeed follow a power law. Quantitatively, however, the correspondence between psychophysics and ecological statistics is poor here: While Oyama (1961) estimated the perceptual power law exponent to be $\alpha \approx$ 2.89, Sigman et al. estimated an ecological exponent of only 0.6, reflective of a much weaker cue to grouping. However, Sigman et al. did not restrict their measurements to pairs of neighboring elements on the same contour of the image. In fact, the measurements were not constrained to be on the same contour, or even on a contour at all, leading to a mixture between strongly related and only weakly related image features. Elder and Goldberg (2002) estimated these distributions more directly, asking human observers to label the sequence of elements forming the contours of natural images, with the aid of an interactive image editing tool. This technique allowed the measurements to be restricted to successive elements along the same contour, and yielded a clear power law with exponent $\alpha=2.92$, very close to the perceptual estimate of Oyama. Whether this exponent is independent of orientation in the image remains an interesting open question, but psychophysical data (Claessens \& Wagemans, 2008) suggest that at least perceptually this may not be the case.

In sum, the convergence between psychophysics and ecological statistics is compelling: Ecologically, proximity follows a power law and exhibits scale invariance, and these properties are mirrored by the psychophysical results. Thus, we have a strong indication that the human perceptual system is optimally tuned for the ecological statistics of proximity cues in natural scenes.

Good continuation. A second important grouping principle for contour integration is good continuation, which refers to the tendency for elements to be grouped to form smooth contours (Wertheimer, 1923/1938c). A very effective method for studying the principle of good continuation in cluttered images was developed by Field, Hayes, and Hess (1993). In this method, a contour formed from localized oriented elements is embedded in a random field of homogeneously distributed distractor elements, in order to eliminate the role of proximity (see Figure 11). Aligning the contour elements tangentially to the contour makes the contour easily detected, whereas randomizing the orientation of the elements renders the contour invisible, clearly demonstrating the role of good continuation in isolation from proximity. These findings led Field et al. to suggest the notion of an association field that determines the linking of oriented elements within a local visual neighborhood (see Figure 12), a construct that is closely related to the machinery of cocircularity support neighborhoods, developed earlier for the purpose of contour refinement in computer vision (Parent \& Zucker, 1989).

Ecological data on good continuation have also emerged over the last decade. Krüger (1998) and later Sigman et al. (2001) found evidence for collinearity, cocircularity, and parallelism in the statistics of natural images. Geisler, Perry, Super, and Gallogly (2001) found similar results using both labeled and unlabeled natural image data, in fairly close correspondence with the tuning of human perception to the good continuation cue. Geisler et al. treated contours as unordered sets of oriented elements, measuring the statistics for pairs of contour elements on a common object boundary, regardless of whether these element pairs were close together or far apart on the object contour. In contrast, Elder and Goldberg (2002) modeled contours as ordered sequences of oriented elements, restricting measurements to adjacent pairs of oriented elements along the contours. The likelihood ratios for two

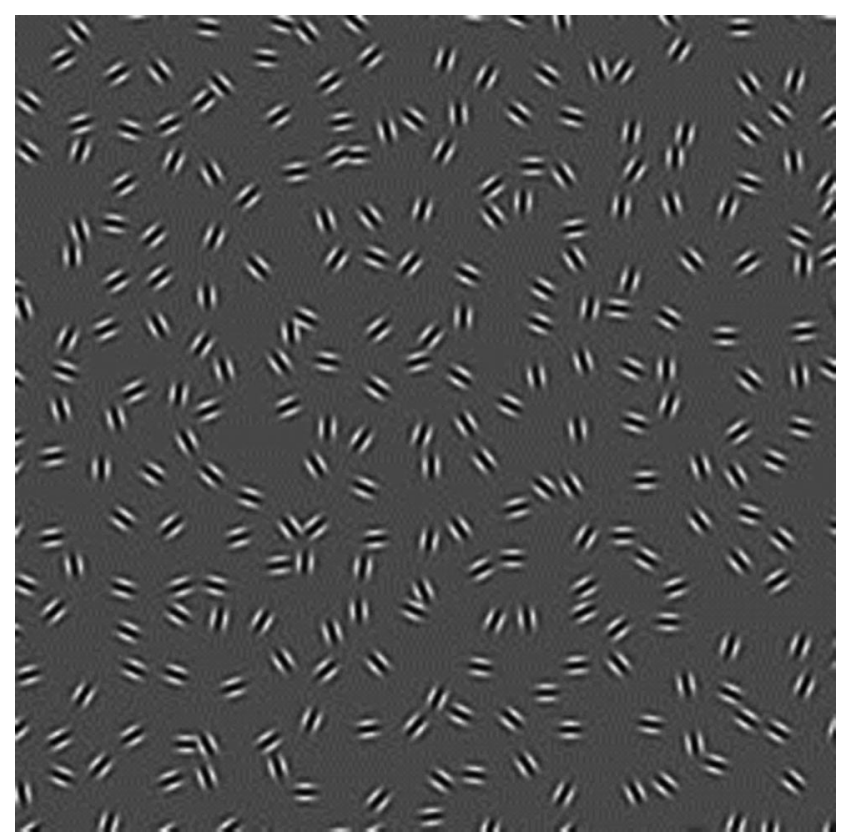

Figure 11. Example of stimuli devised by Field, Hayes, and Hess (1993) to probe the role of good continuation in contour integration. Adapted from "Contour Integration by the Human Visual System: Evidence for a Local 'Association Field'," by D. J. Field, A. Hayes, and R. F. Hess, 1993, Vision Research, 33, p. 177. Copyright 1993 by Elsevier. Adapted with permission. 


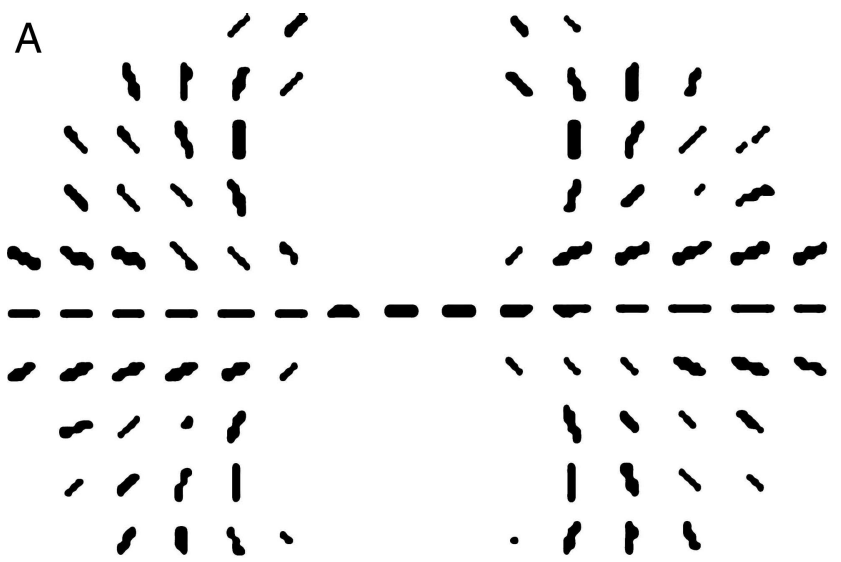

B

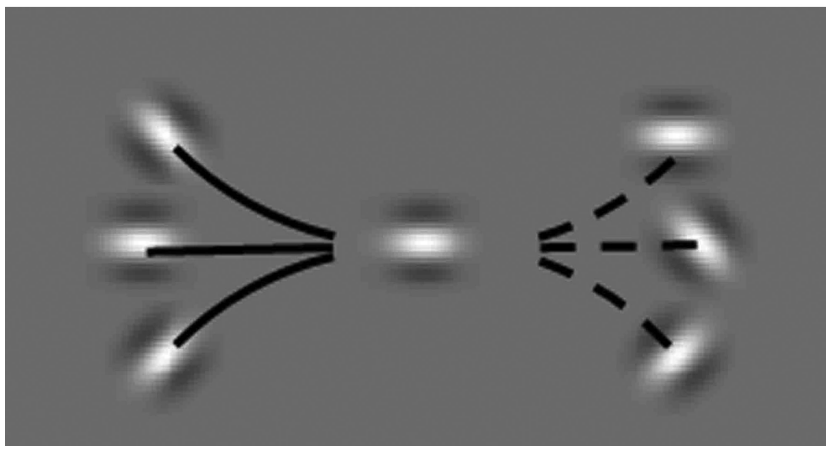

Figure 12. Models of good continuation. A: Cocircularity support neighborhood. B: Association field. Panel A adapted from "Trace Inference, Curvature Consistency, and Curve Detection," by P. Parent and S. W. Zucker, 1989, IEEE Transactions on Pattern Analysis and Machine Intelligence, 11, p. 831. Copyright 1989 by IEEE. Adapted with permission. Panel B adapted from "Contour Integration by the Human Visual System: Evidence for a Local 'Association Field'," by D. J. Field, A. Hayes, and R. F. Hess, 1993, Vision Research, 33, p. 190. Copyright 1993 by Elsevier. Adapted with permission.

oriented elements to be neighboring elements on the same object boundary are much larger for the sequential statistics, reflecting a stronger statistical association between neighboring contour elements.

Similarity. The principle of similarity states that elements with similar properties (e.g., brightness, contrast, color, texture) are more likely to group than elements that differ on these dimensions, which has been demonstrated in a number of ways with dot patterns (see above). For oriented elements, studies have generally found a decline in contour integration performance for contrast reversals under certain conditions but not others, suggesting nonadditive interactions with other grouping cues such as proximity and good continuation (e.g., Elder \& Zucker, 1993; Field, Hayes, \& Hess, 2000; Gilchrist, Humphreys, Riddoch, \& Neumann, 1997; Rensink \& Enns, 1995; Spehar, 2002). Elder and Goldberg (2002) explored the ecological statistics of similarity in edge grouping, coding similarity in terms of the difference in brightness and in contrast between the edges, and found that the brightness cue carries useful information for grouping but the contrast cue is relatively weak. Whereas Elder and Goldberg restricted their study to pairs of elements of the same contrast polarity, Geisler and Perry (2009) have more recently studied the ecological statistics of contrast polarity, demonstrating that it is also an informative cue for contour integration.

Closure. The role of closure in contour integration has been debated. Whereas Kovács and Julesz (1993) found superior detection performance for closed, roughly circular contours, compared to open curvilinear controls, these findings might also be based, in part, on good continuation alone. In fact, with stimuli more closely controlled for good continuation cues, Tversky, Geisler, and Perry (2004) found only a small advantage for closed contours and argued that this advantage could be due to probability summation rather than closure. While this negative result may seem inconsistent with the emphasis placed on closure in early Gestalt work, it is important to appreciate the exact nature of this early view, as expressed by Koffka (1935, p. 150):

\footnotetext{
Ordinary lines, whether straight or curved, appear as lines and not as areas. They have shape, but they lack the difference between an inside and an outside. . If a line forms a closed, or almost closed, figure, we see no longer merely a line on a homogeneous background, but a surface figure bounded by the line.
}

The original Gestalt claim was thus not that closure is a grouping cue per se, but rather that it somehow profoundly determines the final percept of form. In the same spirit, Elder and Zucker (1993, 1994) argued that the most important role for closure was as a bridge from 1-D contour to 2-D shape, a suggestion that was supported by the finding that small changes in good continuation and closure can yield large changes in shape discriminability.

Symmetry and parallelism. The Gestaltists identified symmetry as a factor of good shape (Koffka, 1935), although it seems to be easily overruled by good continuation and convexity (Kanizsa, 1979). In the computer vision literature, symmetry has been used in numerous contour integration algorithms (e.g., Mohan \& Nevatia, 1992; Stahl \& Wang, 2008). Parallelism has been identified as a factor determining the perceptual simplicity of line configurations (Arnheim, 1967), and as a grouping cue in computer vision algorithms (e.g., Jacobs, 2003; Lowe, 1985). In the ecological statistics literature, parallelism has been studied extensively as a cue for the grouping of oriented edge elements into contours (Elder \& Goldberg, 2002; Geisler et al., 2001; Krüger, 1998). Psychophysical evidence for the role of symmetry and parallelism in contour integration has been reported by Feldman (2007), who showed that comparison of features lying on pairs of line segments is significantly faster if the segments are parallel or mirror symmetric, suggesting a fast grouping of the segments based upon these cues. More recently, Machilsen, Pauwels, and Wagemans (2009) demonstrated enhanced detectability of bilaterally symmetric versus asymmetric closed forms, suggesting a role for more complex, global symmetry processing in contour integration. Physiologically, it is known that bilaterally symmetric patterns differentially activate human extrastriate visual areas V3, V4, V7 and LO, and homologous areas in macaque cortex (Sasaki, 2007).

Convexity. Convexity has long been known as a grouping cue (Rubin, 1927). In the computer vision literature, Jacobs (1996) also demonstrated its utility for grouping contour fragments that can then be used as features for object recognition. Liu, Jacobs, and Basri (1999) subsequently employed a novel psychophysical 
method to demonstrate the role of convexity in contour integration. Their method relies on the observation of Mitchison and Westheimer (1984) that judging the relative stereoscopic depth of two contour fragments becomes more difficult when the fragments are arranged to form a configuration with good continuation and closure. Using an elaboration of this method, Liu and colleagues showed that stereoscopic thresholds are substantially higher for occluded contour fragments that can be completed to form a convex shape, relative to fragments whose completion induces one or more concavities. This suggests that the visual system is using convexity as a grouping cue.

In sum, research on contour integration, using oriented elements or shape fragments, has demonstrated important roles for the principles of proximity, good continuation, similarity, closure, symmetry, and parallelism. This research thus can be seen as an elaboration of early Gestalt work that established these principles phenomenologically. Importantly, however, work on contour integration has provided quantitative measures that have led directly to computational models and algorithms, and that have allowed direct comparison to the underlying ecological statistics of the problem and to neurophysiological results.

Whereas the preceding section discussed research on the integration of contour fragments, the next section turns to the specific problem of contour completion in cases of occlusion, which brings us another step closer to understanding the role of grouping in more complex tasks of natural vision.

\section{Contour Completion}

Modal and amodal completion. Whereas contour integration refers to the integration of discrete elements such as dots or oriented elements, contour completion refers to the integration of smooth extended contours, separated by gaps due to occlusion or camouflage. Stimuli used in the study of contour completion often include cues to relative depth or occlusion, such as T junctions (see Figure 13). In Figure 13A, for example, the perceived unity of the black fragments and the perceived shape of the black surface behind the gray occluder appear to result from visual contour completion. This form of completion of the black shape behind the gray occluder is referred to as amodal completion (Michotte et al., 1964). Although one has a compelling sense of continuity of the boundaries of the black surface behind the occluder, one does not actually see a contour. By contrast, illusory contours constitute an example of modal completion. They generate a percept of a contrast border in image regions that are physically homogeneous, such as the illusory edges of the white wedge in Figure 13B.

Grouping and shape problem. For contour completion, the visual system must solve two problems (see Figure 14). First, it must determine whether or not the two contour fragments are part of a single continuous contour: the grouping problem. Second, it must determine the shape of the missing portion of the contour: the shape problem.

As a solution to the grouping problem, Kellman and Shipley (1991) proposed a relatability criterion. This criterion refers to the coding of contour geometry in terms of the positions and orientations of the edges of the contours. Two inducing contours are grouped if they satisfy two conditions. First, if extended linearly, these extensions must intersect, and second, the turning angle of the two edges should not exceed $90^{\circ}$. Evidence for the role of contour relatability has been found by means of observer ratings (Kellman \& Shipley, 1991), shape (fat/thin) discrimination performance (Ringach \& Shapley, 1996; J. Zhou, Tjan, Zhou, \& Liu, 2008), depth discrimination performance (Liu et al., 1999; Yin, Kellman, \& Shipley, 2000), and interpolation settings (Fulvio, Singh, \& Maloney, 2008).

The shape problem involves determining the shape of the occluded portion of the contour. This is a highly underconstrained problem, since an infinite number of possible shapes could be hidden behind the foreground shape (see Figure 14C). Human observers typically perceive only a small subset of these. This suggests that the visual system imposes strong constraints when generating and selecting the set of possible shapes. Different methods have been used to examine how the visual system solves the shape problem. For example, several studies have asked participants to draw the perceived trajectory of visually completed
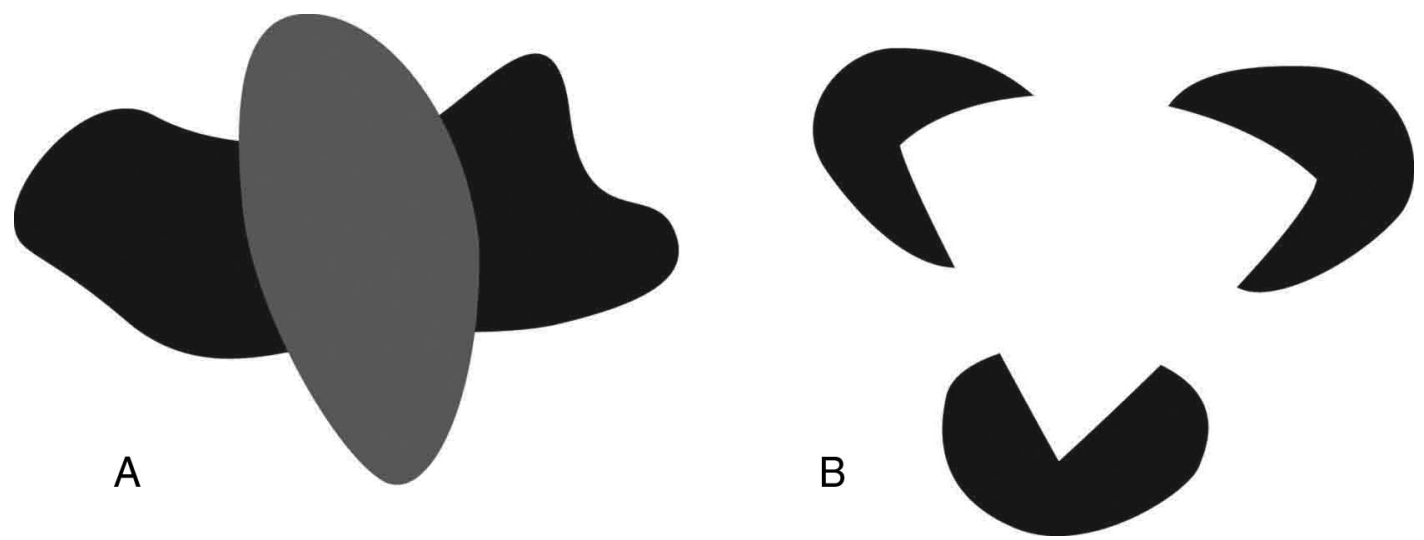

Figure 13. A: Amodal completion of the black shape behind the gray shape. B: A white shape seen on top of three black shapes. The perceived contours of this white shape have a sensory quality (hence, modal completion), although they are completely illusory. Adapted from "Bayesian Contour Extrapolation: Geometric Determinants of Good Continuation,” by M. Singh and J. M. Fulvio, 2007, Vision Research, 47, p. 784. Copyright 2007 by Elsevier. Adapted with permission. 

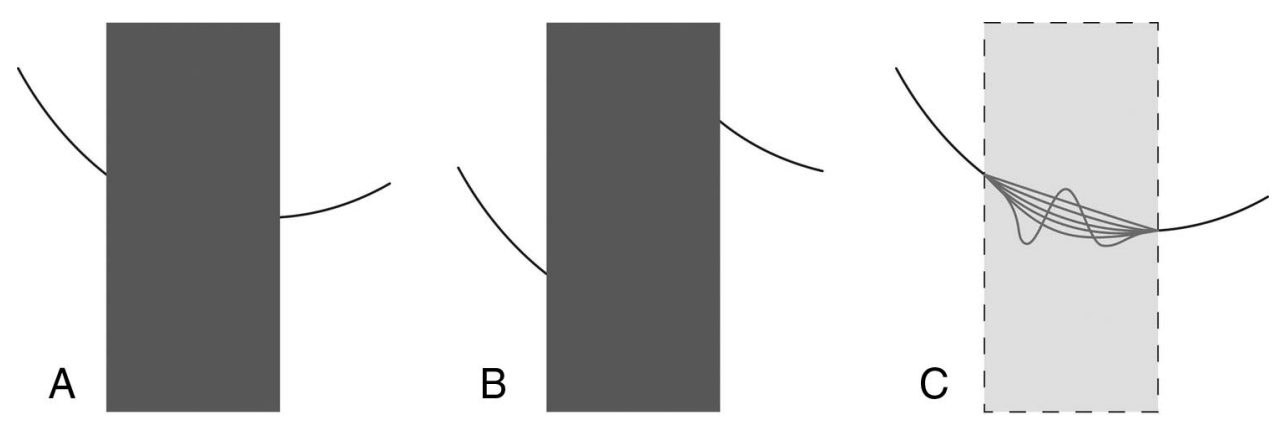

Figure 14. Two curved fragments are seen to complete amodally behind the gray rectangle in Panel A, not in Panel B. C: In case of amodal completion, an important issue regards the shape of the completed curve. Adapted from "Bayesian Contour Extrapolation: Geometric Determinants of Good Continuation," by M. Singh and J. M. Fulvio, 2007, Vision Research, 47, p. 784. Copyright 2007 by Elsevier. Adapted with permission.

contours (Takeichi, Nakazawa, Murakami, \& Shimojo, 1995; Ullman, 1976). Other studies have asked observers to mark the farthest point along the interpolated contours (Fantoni, Bertamini, \& Gerbino, 2005; Guttman \& Kellman, 2004; Takeichi et al., 1995), to match the perceived interpolated shape from a parametric family (Singh, 2004), or to rate its overall degree of roundedness (Fantoni \& Gerbino, 2003). More recently, Fulvio et al. (2008) used a method that obtains interpolation settings (position and orientation) at multiple locations along the length of a partly occluded contour. An examination of the precision and consistency of these settings showed that observers can interpolate a single, stable (self-consistent), smooth contour when the inducing contours are relatable, but not when they require an inflecting interpolating curve.

An empirically successful model that addresses the grouping problem and the shape problem simultaneously has been proposed by van Lier (1999; van Lier, van der Helm, \& Leeuwenberg, 1994, $1995)$. In line with the descriptive simplicity principle (see Wagemans et al., 2012, Sections 5 and 6), this model postulates that an interpretation is judged not only by the complexities of the potentially occluding and occluded shapes but also by the complexity of the relative positions of these shapes. The combination with the lowest overall complexity is then taken to predict whether occlusion occurs, and if so, how the occluded shape looks.

Contour interpolation and extrapolation. Good continuation is an important criterion in shape interpolation, but it is not perfectly clear what is meant by this term. Dating back to Wertheimer (1923, especially his Figures 16-19), two aspects have been distinguished: (a) What geometric properties of the visible contours are used by human vision (e.g., orientation, curvature, rate of change of curvature, higher order derivates), and (b) how are they combined to define the shape of the contour?

These questions were addressed in studies of contour extrapolation (e.g., Singh \& Fulvio, 2005, 2007), in which observers adjusted the position and orientation of a line probe on the other side of an occluder (see Figure 15) at multiple distances from the point of occlusion in order to optimize the percept of smooth continuation. The findings suggested that observers use contour orientation and curvature, but not rate of change of curvature. The shape of visually extrapolated contours was best characterized by a decaying curvature. The results were modeled by a Bayesian interaction between a tendency to extend contour curvature and a prior tendency for straight contours.
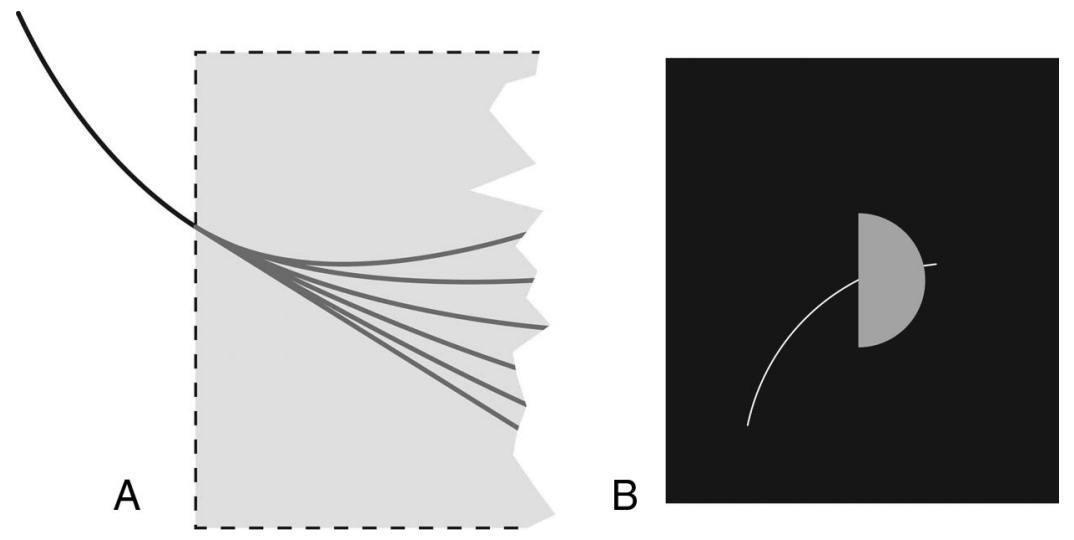

Figure 15. A: Measuring extrapolation of curvature by (Panel B) asking observers to position and orient a small curved line fragment. Adapted from "Bayesian Contour Extrapolation: Geometric Determinants of Good Continuation," by M. Singh and J. M. Fulvio, 2007, Vision Research, 47, pp. 784, 787. Copyright 2007 by Elsevier. Adapted with permission. 
Models of contour interpolation typically use just the position and orientation of the visible segments at their respective points of occlusion, but not their curvature (e.g., Fantoni \& Gerbino, 2003; Kellman \& Shipley, 1991; Ullman, 1976). The above results suggest, however, that interpolation models should use curvature as well, especially if they aim to model human contour interpolation. How much of the visible contours the visual system uses is still an open question. More flexible behavior can be obtained (in terms capturing various dependencies exhibited by human vision) using probabilistic models of contour interpolation rather than those based on fixed classes of shapes, or that minimize fixed geometric criteria (see Singh \& Fulvio, 2007).

Surface geometry and layout. The dominant approach in contour completion has been to treat the problem in isolation (e.g., Kellman \& Shipley, 1991), but there is growing evidence that visual contour completion is very much informed and guided by surface geometry and surface layout.

One aspect of surface geometry is curvature polarity, where a curved contour segment (see Figure 16A) can correspond to a locally convex (see Figure 16B) or a concave portion of a surface (see Figure 16C). In addition, curvature on an object boundary can arise either because the axis of the object itself is curved (like a bending ribbon; see Figure 16D), or because of the changing width of the shape around a straight axis (a convex bulge or a concave neck; see Figures 16B-16C). Experimental studies have shown that the convexity of the surface influences grouping in both contour completion (Liu et al., 1999; Tse, 1999) and interpolated shape (Fantoni et al., 2005; Fulvio \& Singh, 2006). Moreover, the influence of surface geometry goes beyond local convexity to include skeletal shape description (Fulvio \& Singh, 2006).

In addition to surface geometry, surface layout also exerts an influence on contour completion, for example, by differences in perceived depth. In amodal completion, the completed contour tends to belong to the occluded surface. In modal completion, in contrast, it belongs to a nearer, occluding surface. Such effects of surface layout are not consistent with a single mechanism underlying both modal and amodal completion (Anderson, Singh, \& Fleming, 2002; J. Zhou et al., 2008). Instead of assuming two separate mechanisms for modal and amodal completion, however, it could also be assumed that the contour interpolation mechanism is flexible, in that it can take into account various image conditions (e.g., relative depth, photometric conditions, as well as surface geometry; Singh, 2004; J. Zhou et al., 2008). The exact mechanism underlying modal and amodal completion has been the topic of much recent debate (e.g., Anderson, 2007; Kellman, Garrigan, Shipley, \& Keane, 2007).

In sum, when we explicitly study contour completion under occlusion, some additional issues arise, and research indicates that the mechanisms take more complex geometric aspects into account, that they are more sensitive to the configuration in which the visible contour fragments occur, and that they are generally more flexible than in cases of contour completion without occlusion.

\section{Some General Issues Regarding Perceptual Grouping and Contour Integration}

While our review has thus far considered each individual Gestalt principle in turn and how these principles apply to contour integration and completion, there are key overarching questions that cut across all of these cases of perceptual grouping: (a) To what extent are the Gestalt laws innate or learned, (b) how are they combined, and (c) how can they be jointly represented in accurate computational models and useful algorithms?

Development. In general, Gestalt psychology has tended to emphasize the degree to which the Gestalt laws are innate or intrinsic to the brain rather than learned from past experience. Research suggests that infants are capable of grouping visual elements into unitary structures in accord with a variety of both classic and modern organizational principles (for a review, see Bhatt \& Quinn, 2011). Infants as young as 3 to 4 months of age show grouping by good continuation, proximity, connectedness, and common region, and grouping by lightness similarity was observed even in newborns. However, only 6- to 7-month-olds appear to utilize form similarity to organize visual patterns (Quinn \& Bhatt, 2006), and 3-month-olds appear to be completely insensitive to closure (Gerhardstein, Kovács, Ditre, \& Feher, 2004),
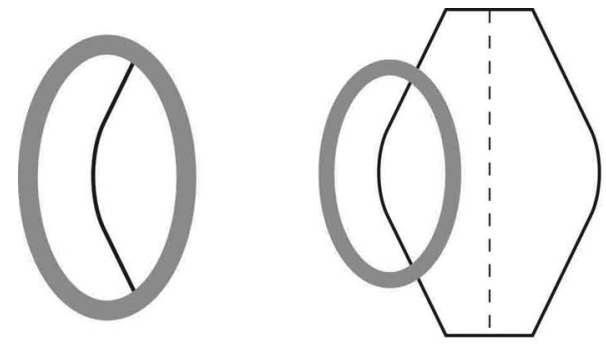

A

B
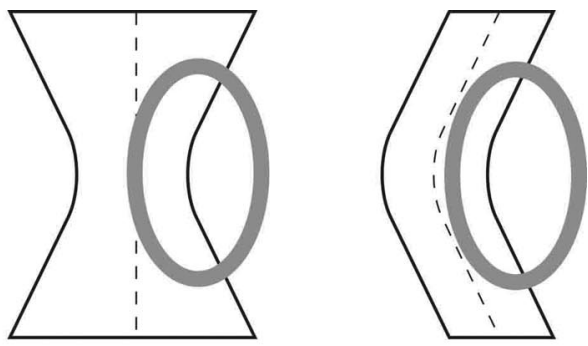

C

Figure 16. Contour geometry depends on surface geometry. The same curved contour segment (Panel A) can correspond either to a locally convex (Panel B) or a concave portion of a surface (Panel C). Curvature on an object boundary can also arise because the axis of the object itself is curved (Panel D). Adapted from "Surface Geometry Influences the Shape of Illusory Contours," by J. M. Fulvio and M. Singh, 2006, Acta Psychologica, 123, p. 23. Copyright 2006 by Elsevier. Adapted with permission. 
suggesting that not all grouping cues are readily available to young infants.

Furthermore, recent psychophysical studies of older children suggest that there is a protracted developmental trajectory for some perceptual organization abilities, even those that appear to emerge during infancy (e.g., Hadad \& Kimchi, 2006; Hadad, Maurer, \& Lewis, 2010a, 2010b; Kimchi, Hadad, Behrmann, \& Palmer, 2005; Kovács, 2000). For example, the Gestalt principles underlying contour grouping continue to develop in children through their late teens. These findings suggest that visual experience plays a role in at least some aspects of perceptual organization. Further support for this notion comes from studies demonstrating influence of perceptual learning on organizational abilities in infancy (e.g., Quinn \& Bhatt, 2005), and the influence of associative learning (Vickery \& Jiang, 2009) and past experience (Kimchi \& Hadad, 2002) on perceptual grouping in adult observers.

Cue combination. One of the central questions in grouping concerns the way in which the brain combines multiple cues to yield a unitary organization. Historically, this problem has often been posed in terms of competitive interactions formulated either in descriptive terms (usually seeking compliance with the simplicity principle) or in probabilistic terms (usually Bayesian formulations that may or may not seek compliance with the Helmholtzian likelihood principle). (For more details on this, see Wagemans et al., 2012, Sections 5 and 6.) However, in natural scenes, disparate weak cues can often combine synergistically to yield strong evidence for a particular grouping.

To explore this issue, Geisler et al. (2001) used a nonparametric statistical approach, jointly modeling the ecological statistics of proximity and good continuation cues as a 3-D histogram, to show that human observers combine these two classic Gestalt principles in a roughly optimal way. Elder and Goldberg (2002) demonstrated that the ecological statistics of proximity, good continuation, and similarity are roughly uncorrelated, so that to a first approximation the Gestalt laws can be factored: The likelihood of a particular grouping can be computed as the product of the likelihoods of each grouping factor in turn (or, equivalently, the log likelihood of the grouping factor is the sum of the log likelihoods of the grouping factors; for further explanation, see Claessens \& Wagemans, 2008).

Elder and Goldberg's (2002) approach also allowed quantification of the statistical power of each Gestalt cue, as the reduction in the entropy of the grouping decision given each cue individually. They found that proximity was by far the most powerful, reducing the entropy by roughly $75 \%$, whereas good continuation and similarity reduced entropy by roughly $10 \%$ each. The most accurate grouping decisions could be made by combining all of the cues optimally according to the probabilistic model, trained on the ecological statistics of natural images. Such a statistically optimal combination of grouping cues has also received some psychophysical support (Claessens \& Wagemans, 2008).

Computational models. Numerous computer vision algorithms for grouping make use of the Gestalt factors described above (e.g., Elder et al., 2003; Estrada \& Elder, 2006; Jacobs, 1996; Lowe, 1985; Sha'ashua \& Ullman, 1988; Stahl \& Wang, 2008). Most of these use the local Gestalt principles of proximity, good continuation, and similarity to group long chains under an explicit or implicit Markov assumption, while additional global factors of convexity (Jacobs, 1996), closure (Elder et al., 2003), and symmetry (Stahl \& Wang, 2008) have been employed to further condition the search. In addition, coarse-to-fine feedback techniques have been used as an alternative way to incorporate more global constraints into the grouping process (Estrada \& Elder, 2006). Grounding probabilistic algorithms in the ecological statistics of contour grouping avoids the ad hoc selection of algorithm parameters and optimizes performance on natural scenes (Elder et al., 2003; Estrada \& Elder, 2006).

Although the Gestalt principles of grouping were largely based on the analysis of figures in the 2-D image plane, more recent work derives these principles from the geometric laws of 3-D projection, within the theoretical framework of minimal viewpoint invariants (Jacobs, 2003; Lowe, 1985). Briefly, the theory is based upon the assumption that the observer takes a general viewpoint position with respect to scenes. This assumption implies that certain socalled nonaccidental properties in the 2-D proximal stimulus are most likely properties of the 3-D distal stimulus as well (Binford, 1981; Lowe, 1985). Examples of such nonaccidental properties are the geometric Gestalt principles relevant to contour grouping: proximity, good continuation, closure, convexity, parallelism, and symmetry (e.g., Wagemans, 1992, 1993). This notion has played a central role as a bridge between the Gestalt grouping principles and object representation and recognition (Biederman, 1987).

\section{Conclusion}

Research on principles of perceptual grouping has come a long way since Wertheimer's (1923) phenomenological demonstrations. Using well-controlled discrete patterns, grouping by proximity could be quantified as a real law. In the last 2 decades or so, new grouping principles have been identified, and their functional role in the processing of more complex images occurring in real-world situations has become a central focus. In much of this research, quantitative psychophysical work went hand in hand with the analysis of ecological statistics, the development of computational models, and neurophysiological research. In Section 5, we see a similar evolution in research on the closely related problem of figure-ground organization. Section 6 is devoted to an integrated review of the research on neural mechanisms of contour grouping and figure-ground organization.

\section{Figure-Ground Organization}

\section{Introduction}

Two adjoining regions of the visual field with a shared border can lead to a mosaic percept, but more often lead to figure-ground organization. In this percept, the shared border is perceived as the occluding edge of one of the regions. The occluding region is perceived as the figure, and it appears shaped by the border. The adjoining region appears to simply continue behind it as its background, with no shape being imparted by the border. The figure is said to own the borderline. When figure-ground reversals occur, the border ownership switches as well (e.g., the famous vase-faces figure by Rubin, 1915). Why switching occurs and how its temporal characteristics inform us about the dynamics of the brain are discussed extensively in the second article (Wagemans et al., 2012, Section 3). Here, we focus on the factors or principles that determine what is perceived as figure. 
The question of whether or not experience plays a role has been particularly controversial. On the one hand, structuralists like Wundt argued that past experience was the sole determinant of which region of the visual field was perceived as figure. On the other hand, Gestalt psychologists such as Wertheimer, Koffka, and Köhler considered the structuralist position untenable because it would draw too strongly on memory to be useful for real-time perception. They also pointed out that novel objects can be perceived easily, which is inconsistent with a theory in which only past experience causes perceptual organization. The Gestalt view was that figure-ground segregation occurs by innate, intrinsic segregation laws that embody aspects of the world in which human perception evolved. The resultant shaped entities (figures) are then matched with traces in memory, a process that requires a less extensive memory search. In this view, familiar and novel objects are distinguished only after they attain figural status.

Wertheimer argued that past experience was unlikely to influence initial organization. He put forward two requirements to be met for past experience to convincingly play a role in figureground perception (Wertheimer, 1923/1938c, p. 86): “[The] duty [of the doctrine of past experience] should be to demonstrate ... (1) that the dominant apprehension [perception] was due to earlier experience (and to nothing else); (2) that nondominant apprehensions in each instance had not been previously experienced." The Gestalt psychologists demonstrated that a variety of image properties were sufficient for the perception of figures or configurations, without the need of familiarity. These image propertiesconvexity, symmetry, small area, and surroundedness (or enclosure)- became known as the classic configural principles of figure-ground organization (e.g., Harrower, 1936; Rubin, 1915).

\section{Classic Image-Based Configural Principles of Figure-Ground Organization}

Traditionally, investigators have tested the effectiveness of configural factors in controlled experiments, using displays composed of multiple alternating black and white regions where either the black or the white region could have the configural factor (e.g., convexity in Figure 17). Participants were asked to indicate which region, the black or the white one, was perceived as the figure. These experiments support less formal early demonstrations of the importance of configural factors for initial figure-ground segregation, without the need to rely on past experience (familiarity). The most powerful demonstrations were obtained with convexity and symmetry, although these principles were often confounded in earlier studies (e.g., Bahnsen, 1928). Confounding often occurred in tests of the other two configural principles-small region and surroundedness-as well, which created some confusion on the individual effectiveness of each of these principles. Note that long exposure times were used in these early demonstrations, so it is not clear that reports reflected initial organization. Later, more controlled investigations of convexity and symmetry revealed that their strength was overestimated by traditional demonstrations.

Convexity. Also using long exposures, Kanizsa and Gerbino (1976) tested the effectiveness of convexity as a configural figureground principle, using displays similar to Figure 17, and found that regions with convex parts were seen as figure on approximately $90 \%$ of trials. These results - in tandem with the previous Gestalt demonstrations-were taken as evidence that part convexity might determine to a large extent the position of figures in the input array, even before memories of previously seen objects are accessed (see Singh \& Hoffman, 2001). To examine whether the effects of part convexity are general in that they extend to stimuli with fewer regions than used in the original displays, Peterson and Salvagio (2008) presented displays that could contain either two, four, six, or eight regions. In addition, the exposure duration was reduced to $100 \mathrm{~ms}$. Interestingly, the likelihood of perceiving convex regions as figures increased from only $57 \%$ of trials with two-region displays up to $89 \%$ for eight-region displays (as in Kanizsa \& Gerbino, 1976). This effect of the number of alternating regions - the convexity context effect—was obtained only when the concave regions were homogeneous in color, whereas the color of the convex regions was irrelevant. Thus, homogeneity of alternating regions functions as a background cue, but other experiments indicated that it does so only when a configural cue favors
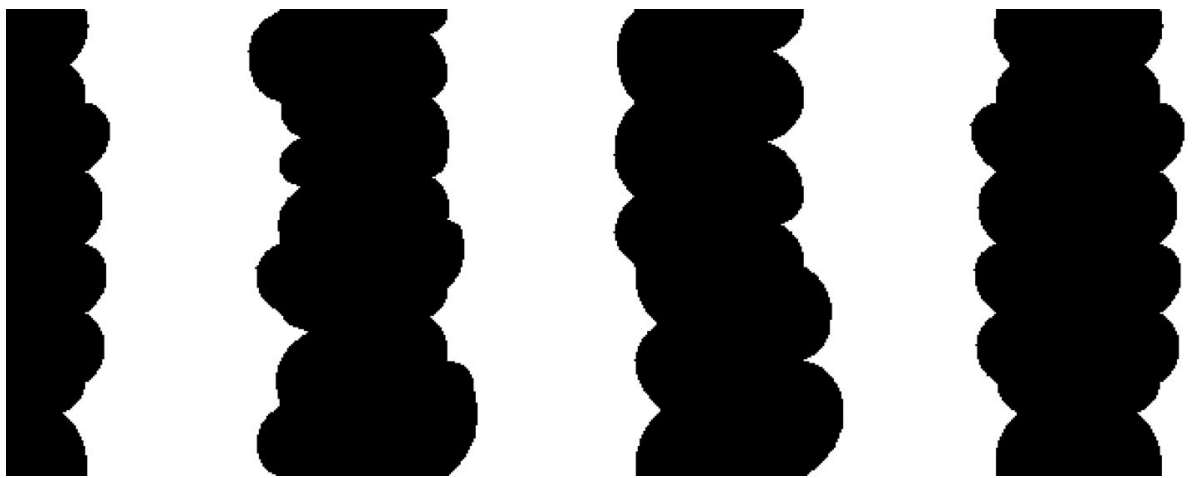

Figure 17. Example of a display used in classic tests of whether or not convexity serves as a configural figure-ground principle. Here the black regions have convex parts, and the white regions have concave parts. Regions with convex parts were black in half of the test displays and white in the other half. Adapted from "Inhibitory Competition in Figure-Ground Perception: Context and Convexity," by M. A. Peterson and E. Salvagio, 2008, Journal of Vision, 8(16), Article 4, p. 2. Copyright 2008 by the Association for Research in Vision and Ophthalmology. Adapted with permission. 
perceiving the regions in-between as figures. These experimental observations are consistent with a Bayesian ideal observer model that estimates the probability that a picture depicts a 3-D scene or a 2-D pattern (Goldreich \& Peterson, 2012). Specifically, to be able to fit Peterson and Salvagio's data, the Bayesian observer had weak biases for convex over concave objects and for single-color over multicolor occluded objects.

Symmetry. Kanizsa and Gerbino (1976) measured the effectiveness of global symmetry as a configural figure-ground principle by placing it in competition with convexity. They found that convex-region-as-figure reports were unaffected by competition from symmetry, suggesting that symmetry is only a weak factor (see also Pomerantz \& Kubovy, 1986). However, with longer exposures of eight-region displays consisting of alternating symmetric and asymmetric regions (with no competition from convexity), Driver, Baylis, and Rafal (1992) found that symmetric regions were perceived as figures on a large proportion of the trials. When Peterson and Gibson (1994a) examined the effectiveness of symmetry as a configural figure-ground principle in two-region displays with brief exposure durations, their observers reported perceiving the symmetric region as figure on approximately three quarters of the trials. Effects of global symmetry have also been found for shapes defined by a contour of Gabor elements presented in a noisy background (Machilsen et al., 2009), suggesting that global symmetry is effective in segregating figures from backgrounds. Together, these findings are consistent in showing that-at least near fixation-global symmetry is a configural figure-ground principle.

\section{New Image-Based Principles of Figure-Ground Organization}

All of the above classic figure-ground principles concern the organization of displays consisting of static, homogeneously colored regions. Quite a few additional principles of figure-ground organization have since been discovered, some of which also apply to static, homogeneously colored regions (e.g., lower region, and top-bottom polarity). Additional figure-ground principles come into play in displays containing spatial heterogeneities such as texture (extremal edges and edge-region grouping) and motion (advancing region, articulating concavities, and convex motion). Pinna (2010) recently even extended the range of application of these principles to shape and meaning. As with the new grouping principles, researchers have often provided an ecological foundation of these new figure-ground principles too.

Lower region. Vecera, Vogel, and Woodman (2002) showed that when a rectangular display is divided in half by an articulated horizontal border, the region below the border is more likely to be perceived as the closer, figural region than is that above the border. They found this lower region bias most strongly in cityscape images in which the border consisted of horizontal and vertical segments, but also in analogous stalactite/stalagmite images consisting of curved segments. Vecera (2004) performed additional experiments in which such displays were viewed by observers whose heads were tilted (or even inverted) to determine whether this figure-ground bias was driven by a viewer-centered, retinal reference frame or an environmental reference frame. The results showed-somewhat surprisingly-that retinal directions were clearly dominant. Although this result is at odds with the presumed ecological justification of gravity as the rationale for perceiving lower regions as closer (see Vecera \& Palmer, 2006), it is consistent with the need to compute information about figure-ground status early in visual processing, before orientation constancy has occurred. Indeed, because head orientation is approximately vertical most of the time, the difference between retinal and environmental reference frames is negligible. The ecological validity of lower region was assessed statistically by analyzing a corpus of photographic images that were hand-segmented by human observers (Martin, Fowlkes, Tal, \& Malik, 2001). The results showed that lower region was a valid cue to closer surfaces at local edges whose orientation was roughly horizontal. Vecera and Palmer (2006) argued that the effect of seeing lower regions as closer resulted from biases in the ecological statistics of depth edges at various orientations when objects rest on a ground plane beneath them, as do most terrestrial objects in the earth's gravitational field.

Top-bottom polarity. Hulleman and Humphreys (2004) showed that regions that are wider at the bottom and narrower at the top are more likely to be perceived as figures than regions that are wider at the top and narrower at the bottom. The regions in their displays looked a bit like odd evergreen trees or chess pieces, but they argued that their effects were not due to the effects of familiar shape (e.g., Peterson \& Gibson, 1994a) because there are other familiar objects (e.g., tornados) that are similar in shape to the regions with narrow bases and wide tops. They also claimed that top-bottom polarity effects cannot be explained by the effects of lower region (Vecera et al., 2002). Nevertheless, the ecological factor that links all three of these figure-ground factors (canonical orientation of familiar shapes, lower region, and top-bottom polarity) is gravity. Indeed, top-bottom polarity can easily be interpreted as due to the perceptual consequences of gravitational stability.

Extremal edges and gradient cuts. An extremal edge (EE) in an image is a projection of a viewpoint-specific horizon of self-occlusion on a smooth convex surface, such as the straight side of a cylinder. Computational analyses of the visual properties of surfaces near EEs show characteristic gradients of shading and/or texture in which the equiluminance and/or equidensity contours are approximately parallel to the edge of the surface (Huggins \& Zucker, 2001). EEs are relevant to figure-ground determination because the side with an EE gradient is almost invariably perceived as being closer to the observer than the opposite side of the edge (Palmer \& Ghose, 2008), even when the EE is placed in conflict with other factors (Ghose \& Palmer, 2010).

Edge-region grouping. Palmer and Brooks (2008) built a bridge between classic grouping effects and figure-ground organization by reasoning that if figure-ground organization is indeed determined by an edge belonging to (i.e., grouping with) the region on one side more strongly than that on the other, then any grouping factor that could relate an edge to a region should also operate as a figure-ground factor. They tested this hypothesis for six different grouping factors that were well defined for both an edge and a region-common fate, blur similarity, color similarity, orientation similarity, proximity, and flicker synchrony-and found that all six factors showed figure-ground effects in the predicted direction, albeit to widely varying degrees.

Articulating motion. Barenholtz and Feldman (2006) demonstrated a dynamic principle of figure-ground organization: 
When a contour deforms dynamically, observers tend to assign figure and ground in such a way that the articulating (or hinging) vertices have negative (concave) curvature-which ensures that the figure side is perceived as containing rigid parts articulating about their part boundaries. In their experiments, this articulatingconcavity bias was shown to override traditional static factors (such as convexity or symmetry) in cases where they made opposing predictions. In other experiments, Barenholtz (2010) showed that when a contour segment that is concave on one side and convex on the other deforms dynamically, observers tend to assign the figure on the convex rather than the concave side.

Advancing region motion. Barenholtz and Tarr (2009) showed that moving a border within a delimited space such that the bounded area on one side grows larger and the bounded area on the other side shrinks in size causes the growing area to be perceived as a figure advancing onto the shrinking area. Thus, motion in an advancing region overpowers the classic Gestalt factor of small area.

Contour entropy as a determinant of ground or hole. All of the above research aimed at finding factors that determine the perception of a figure against a background. Gillam and Grove (2011) recently asked whether there are also factors that strengthen the perception of a region as ground. They reasoned that occlusion by a nearer surface will usually introduce a regularity among terminations of contours at the occluding edge, which will be perceived as a stronger cue to occlusion when the irregularity of the elements is higher. In other words, when the lines being terminated are more disordered, the strength of the occlusion cue (called order within disorder or entropy contrast) is larger. They predicted that unrelated (high-entropy) lines would tend to appear as ground (or holes) in a figure-ground paradigm more often than more ordered (low-entropy) lines, which was confirmed in three experiments. This work significantly expands earlier work on the perception of holes (e.g., Bertamini, 2006; Bertamini \& Hulleman, 2006; Nelson \& Palmer, 2001).

\section{Nonimage-Based Influences on Figure-Ground Perception}

Past experience. For most of the 20th century, the Gestalt view that past experience did not influence initial figure-ground perception prevailed. Part of the reason for this continued view might have been that few direct tests existed of the influence of past experience on figure-ground perception that satisfied Wertheimer's (1923) criteria mentioned above. Either there was no evidence that past experience alone drove the effect, or it was possible that past experience exerted its influence after the initial perceptual reorganization. Other observer-dependent factors such as perceptual set and attention were also assumed to be high-level influences, operating only after figures had been segregated from grounds. Recently, evidence that past experience can influence figure assignment has accumulated both from direct report and indirect, response time measures.

Direct reports. Using direct report measures, Peterson, Harvey, and Weidenbacher (1991; Peterson \& Gibson, 1994a) found that regions that portrayed portions of familiar objects were more likely to be perceived as figures when they were upright (and hence, portrayed the familiar object in its canonical orientation) than when they were inverted (see Figure 18). Importantly, regions portraying upright, but not inverted, familiar objects were more likely to be obtained as figures by reversing out of the alternative interpretation, suggesting that past experience in the form of familiar configuration exerts an influence on the initial determination of figure and ground (B. S. Gibson \& Peterson, 1994; Peterson et al., 1991; Peterson \& Gibson, 1994a, 1994b).

The stimuli used in these studies most likely satisfied Wertheimer's first criterion for evidence of past experience effects on figure assignment. However, it was-and still is—difficult for experiments that measure subjects' phenomenal reports to satisfy his second criterion (to exclude that past experience exerts its influence after some initial perceptual reorganization). Even with masked exposures as short as $28 \mathrm{~ms}$, such as those used by Peterson and Gibson (1994a), it is still possible that some preliminary, unconscious, figure-ground determination preceded the organization about which observers reported (Epstein \& DeShazo, 1961), introducing the need for an indirect or implicit measure.

Indirect measures. Driver and Baylis (1996) were the first to use reaction time measures to index figure-ground perception in an experiment in which subjects were shown a brief exposure of a display containing a small high-contrast region lying on a larger rectangle. In this stimulus configuration, both contrast and the configural factor of small area biased participants to see the small region as figure (see Figure 19A). On each trial, participants were asked which of two small, enclosed test shapes shown after the initial display had the same border as the stepped border dividing the rectangle into two regions. On half of the test trials, the test shape lay on the same side of the border as the region that was likely to have been perceived as figure (figure probes; see Figure 19B), whereas on the remainder of the test trials, the test shape lay on the opposite side (ground probes; see Figure 19C). Faster and more accurate responses were obtained on figure test trials than on ground test trials, suggesting that the border shared by two regions in the initial display was automatically bound to the one cued as figure. (For later use of Driver and Baylis's indirect measure, see Hulleman \& Humphreys, 2004; Vecera, Flevaris, \& Filapek, 2004.)

Driver and Baylis (1996) also interpreted the results they obtained with their indirect measure to indicate that past experience does not influence figure assignment, reasoning that response times should be equally fast for figure and ground probes if past experience were operating. Peterson and Enns (2005), however, argued that this reasoning was flawed and that a past experience account could also predict the pattern of results obtained on ground test trials by Driver and Baylis (see also Peterson \& Lampignano, 2003). This is because the repetition of the border from the first trial would reinstantiate the memory of where the figure lay when the border was first encountered, and this memory would compete with the current cues favoring the figure on the opposite side of the border. This competition would slow responses on ground test trials compared to figure test trials. Peterson and Enns introduced control test trials to investigate this alternative interpretation. They asked subjects to ignore the first display and then to decide for a second display whether the pair of small closed figures it contained was identical (as a consequence, the first display served as a priming display). Consistent with the past experience account, response times were slower on ground test trials (and faster for figure test trials) compared to control trials facing in the same direction. 


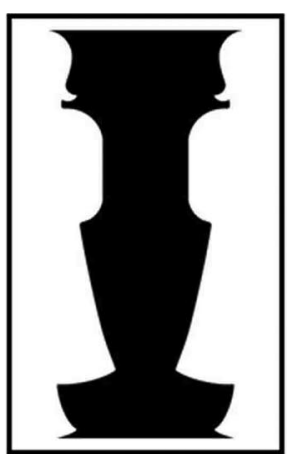

A

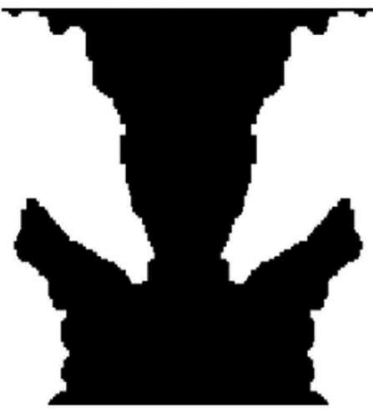

E

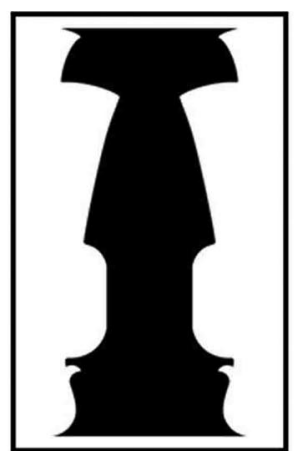

B

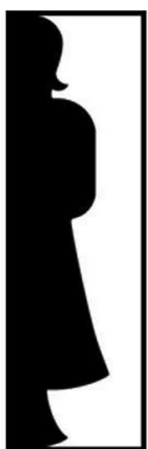

C

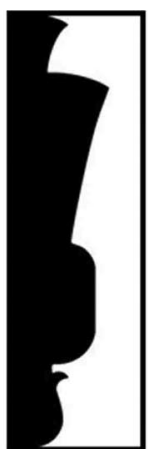

D

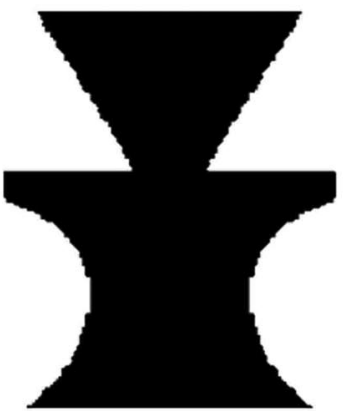

$\mathbf{F}$

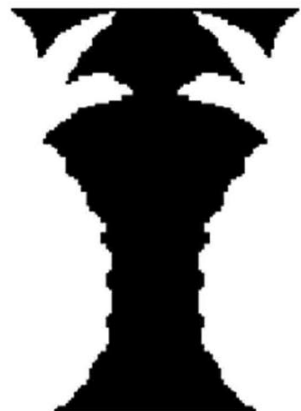

$\mathbf{G}$

Figure 18. A-B: Sample stimuli used by Peterson, Harvey, and Weidenbacher (1991). The configural factors of small area, symmetry, and enclosure favor seeing the central, black region as figure. In Panel A, a portion of a familiar object, a standing woman, is suggested on the outside of the left and right borders of the black region. Panel B is an inverted version of Panel A. C-D: Sample upright (Panel C) and inverted (Panel D) bipartite displays used by Peterson and Gibson (1994a). E-G: Sample stimuli used by Peterson and Skow (2008). The configural factors of small area, symmetry, and enclosure favor seeing the inside of the black silhouettes as the figures. Portions of familiar objects are suggested on the outsides of the silhouettes' left and right borders (in Panel E, sea horses; in Panel F, table lamps; in Panel G, pineapples). Panels A-B adapted from "Shape Recognition Contributions to Figure-Ground Reversal: Which Route Counts?", by M. A. Peterson, E. M. Harvey, and H. J. Weidenbacher, 1991, Journal of Experimental Psychology: Human Perception and Performance, 17, p. 1077. Copyright 1991 by the American Psychological Association. Panels C-D adapted from "Must Figure-Ground Organization Precede Object Recognition? An Assumption in Peril," by M. A. Peterson and B. S. Gibson, 1994, Psychological Science, 5, p. 254. Copyright 1994 by Sage Publications. Adapted with permission. Panels E-G adapted from "Inhibitory Competition Between Shape Properties in Figure-Ground Perception," by M. A. Peterson and E. Skow, 2008, Journal of Experimental Psychology: Human Perception and Performance, 34, p. 254. Copyright 2008 by the American Psychological Association.

Further evidence for the role of past experience in figureground perception was obtained by Vecera and Farah (1997), who presented observers with overlapping outline letters and asked them to determine whether two probed locations were on the same letter or on different letters, a distinction that depended on figureground organization. Congruent with an influence of past experience, faster and more accurate responses were obtained when the outline letters were in their familiar upright orientation rather than inverted. Peterson and Skow (2008) went one step further using displays like those in Figures 18E-18G, in which shape properties such as small area, enclosure, and symmetry strongly favored perceiving the figure on the inside of a silhouette's borders, but past experience favored seeing the figure on the outside. The stronger factors favoring the figure on the inside dominated the competition for figural assignment. Nevertheless, responses to the familiar object that lost the competition were suppressed for a brief period of time after figure-ground perception was achieved, providing evidence that past experience plays a role in figureground segregation even when it does not dominate (see also Trujillo, Allen, Schnyer, \& Peterson, 2010, for a study measuring the associated event related potentials). Recently, Navon (2011) conducted a series of experiments that he took as evidence that the familiarity of objects may exert an influence even earlier, when prefigure assignment parsing occurs.

Taken together, these findings from studies that satisfy both of Wertheimer's (1923) criteria show that past experience can exert an influence on several aspects of figure-ground perception.

Attention and perceptual set. Before any behavioral evidence was obtained that attention affects which region will be perceived as figure, Kienker, Sejnowski, Hinton, and Schumacher 


\section{A. Study Display}

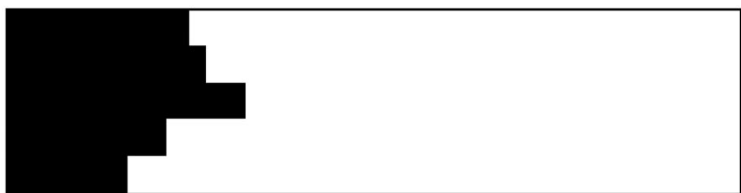

\section{B. Figure Probes}
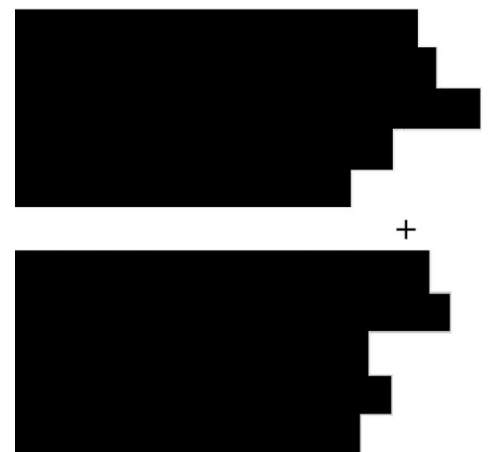

\section{Ground Probes}

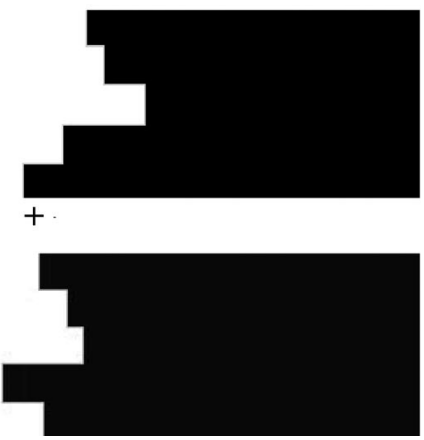

Figure 19. Sample displays used by Driver and Baylis (1996). A: Study display. B-C: Figure and ground probes, respectively. In both Panel B and Panel C, the top probe has the same border as the study display. Adapted from "Edge-Assignment and Figure-Ground Segmentation in Short-Term Visual Masking," by J. Driver and G. C. Baylis, 1996, Cognitive Psychology, 31, p. 254. Copyright 1996 by Elsevier. Adapted with permission.

(1986; Sejnowski \& Hinton, 1987) modeled figure-ground segregation as cross-border inhibitory competition that could be biased by attention. A decade later, Baylis and Driver (1995) presented empirical evidence for the role of attention. They found that when observers allocated their attention endogenously to one of two regions sharing a border, they were more likely to perceive the attended region as figure than the unattended region. Similar but weaker effects of exogenous attention were later obtained (Vecera et al., 2004). In addition, it has been shown that the viewer's intention, or perceptual set, to perceive one of two regions sharing a border as figure can influence figure assignment (Peterson et al., 1991; Peterson \& Gibson, 1994b). Thus, there is ample evidence that observer-dependent factors can influence figure assignment.

The effects of attention and perceptual set contradict claims that figure-ground perception always occurs early in the visual system. Indeed, although figure-ground perception can be affected by focused attention, there is evidence that it can also occur preattentively. Preattentive processes are considered early in that they are carried out before attention is focused on individual regions in the visual field, that is, when attention is either distributed broadly or allocated to a different task. For example, in a case study of a patient with left neglect (a failure to attend to the left side of objects and spaces), it was found that judgments of the symmetry of individual shapes in the left hemisphere were severely impaired (Driver et al., 1992). Nevertheless, when asked to make figureground judgments regarding six-region displays with green and red alternating symmetric and asymmetric regions, the patient, like controls, showed a bias to see symmetric regions as figures, suggesting that the patient could use symmetry preattentively for figure-ground segregation even though he could not detect it postattentively. Convexity-based figure-ground segregation can also occur preattentively. Kimchi and Peterson (2008) asked participants to perform a demanding change detection task on a stimulus presented on a task-irrelevant background of alternating regions of figures and grounds by convexity. They found that changes in the organization of the background produced congruency effects on target-change judgments, even though participants were unable to report the figure-ground status of the background. Taken together, these findings are consistent with the claim that figure-ground segregation can occur before focal attention is directed to making a figure-ground judgment. Thus, figureground segregation can occur preattentively, but it can also be affected by attention.

\section{Figure-Ground Organization in Relation to Shape and Depth Perception}

Traditionally, figure-ground organization has been studied as a relatively isolated, albeit important, aspect of perceptual organization. Studies like those reviewed above, however, have clearly shown that figure-ground organization is closely related to other aspects of visual perception such as shape and depth perception.

Shape perception. Consider the following demonstration due to Attneave (1971): A random wiggly contour is drawn across a disk, dividing it into two, and the two half-disks are spatially separated (see Figure 20). Even though the contour is-by construction-identical on the two halves, it looks different. The wiggly contour on the left half-disk appears to contain three wide, smooth parts, whereas on the right, it appears to contain four narrow, pointy parts. This demonstration suggests that the visual system assigns shape descriptions to surfaces, rather than to the 

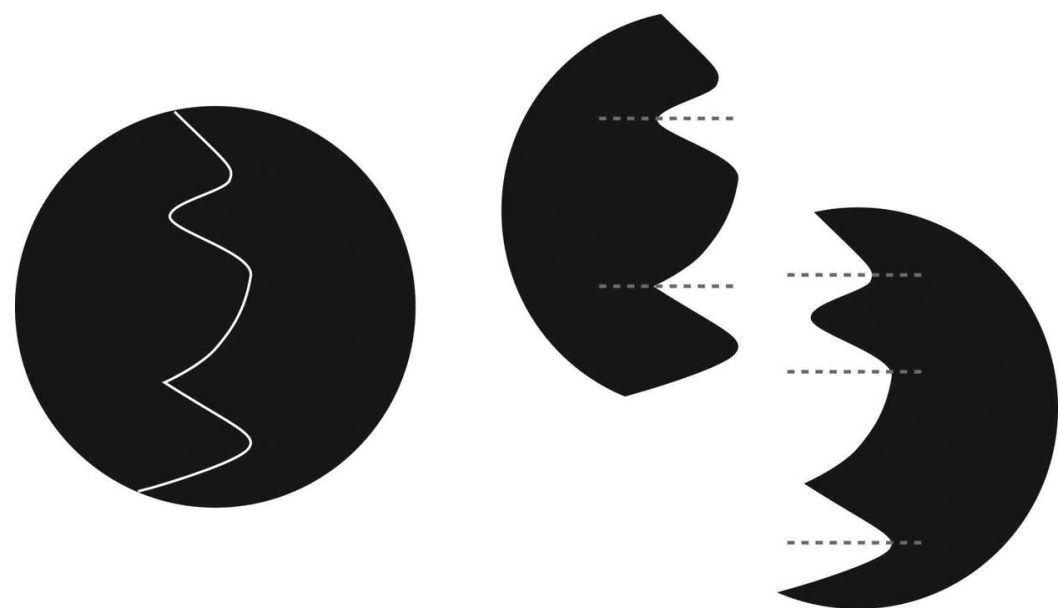

Figure 20. When a wiggly curved line is drawn on a circular disc, the two halves arising from this divide appear to have a bounding contour with a different shape. Adapted from "Multistability in Perception," by F. Attneave, 1971, Scientific American, 225(6), p. 68. Copyright 1971 by Scientific American.

bounding contours of those surfaces: Even when instructed to judge a contour in isolation, observers cannot help but be influenced by the geometry of the surface to which the contour belongs. Hence, shape description is closely tied to figure-ground assignment.

One account of how these processes interact was offered by Hoffman and Richards (1984). They proposed that the visual system segments objects into parts at points of negative minima of curvature (points of locally highest curvature in concave regions) along the bounding contour of the shape. According to this minima rule, the same wiggly contour in Figure 20 is segmented differently on the two half-disks because, when figure and ground switch, so do the roles of convexities and concavities. Hence perceptual part boundaries - which lie in concavities-are located at different points on the two halves (marked with the dots in Figure 20). The contour is perceptually segmented into parts differently on the two half-disks-hence it looks different. This account of part segmentation has been supported in a number of experiments (e.g., Barenholtz \& Feldman, 2006; Baylis \& Driver, 1994; Cohen \& Singh, 2007; De Winter \& Wagemans, 2006; Singh \& Hoffman, 2001).
Building on this account, Hoffman and Singh (1997) proposed that the relative salience of the two sets of parts, one at each side of the borderline, plays an important role in determining figure and ground. (In this respect, it could be considered one of the new image-based principles of figure-ground organization reviewed before.) The perceptual salience of a part, or part salience for short-how much it visually stands out as a separate part-is determined by a number of geometric factors, including the curvature (sharpness) of its part boundaries, and its protrusion (the degree to which it sticks out, measured as perimeter/cut length). Typically, the side with the more salient parts is assigned figural status, as illustrated in Figure 21. In this figure, Panel a shows the original contour, Panel $b$ shows the parts obtained from the two sides, and Panel c shows the side with more salient parts, which tends to be perceived as figure. Because part salience is based on relatively local computations, figure and ground can perceptually reverse along a continuous contour (see Figure 21d). This suggests that shape analysis occurs not only on the figure side-after figureground assignment has taken place-but actually precedes it, and it plays a crucial role in making the figure-ground assign-

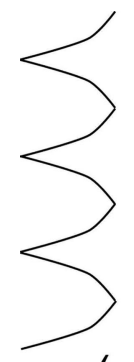

(a)

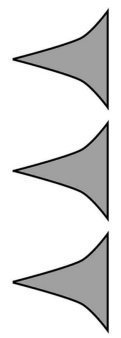

(b)

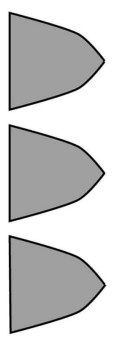

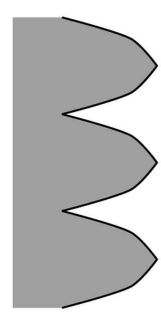

(c)

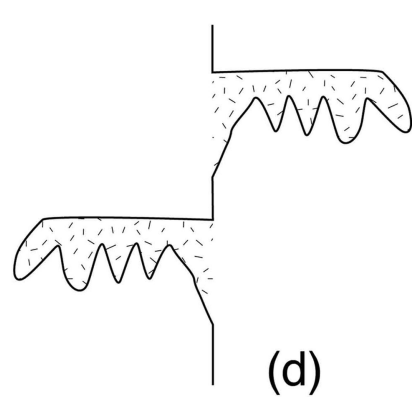

Figure 21. The role of part salience in figure-ground organization. Adapted from "Salience of Visual Parts," by D. D. Hoffman and M. Singh, 1997, Cognition, 63, p. 47. Copyright 1997 by Elsevier. Adapted with permission. 
ment. This account is consistent with previous proposals made by Peterson and colleagues discussed before, although it involves only low-level shape analysis, and does not posit access to memories of familiar shapes.

Another way of thinking about this shape analysis is in terms of a skeletal or axial representation of shape. (Hence, axiality could also be added to the list of new image-based principles of figureground organization.) Skeletal or axial representations provide a compact stick-figure representation that reflects the qualitative branching structure of a shape (Blum, 1973; Leyton, 1989; Marr \& Nishihara, 1978). In a recent probabilistic model of shape skeletons (Feldman \& Singh, 2006), the goodness of a skeletonformally, its posterior pr(SkeletonlShape)-is evaluated in terms of (a) how well the skeleton explains the shape-its likelihood $\operatorname{pr}($ ShapelSkeleton) — and (b) its simplicity - the prior pr(Skeleton), which embodies a preference for fewer branches and less curved branches. The best skeleton (that maximizes the posterior) provides an intuitive summary description of the shape, and allows a one-to-one correspondence between its skeletal branches and the parts of the shape. The skeletal model of shape description applies to the figure-ground context as follows: The skeleton is computed from both sides of the contour, yielding skeletal descriptions of the surface on either side; and whichever side has a more axial shape (higher posterior) wins the figure-ground competition. A recent Bayesian implementation of this competition has been shown to closely resemble human perception of figure and ground (Froyen, Feldman, \& Singh, 2010).

Depth perception. Because the figure is by definition closer to the observer than the ground, figure-ground organization is necessarily related to certain aspects of depth perception. One can think of figure-ground principles as a subset of sources of depth information that apply to the special case of inferring relative (i.e., ordinal) depth across an edge. Recently, the seemingly ordinal figure-ground factor of convexity was found to combine with classic metric depth cues, such as binocular disparity (Burge, Peterson, \& Palmer, 2005), suggesting that it might be a metric rather than an ordinal depth cue. Indeed, in a recent study of natural scene statistics, Burge, Fowlkes, and Banks (2010) concluded that convexity is a metric cue to depth. Many questions remain regarding how figural cues signal depth.

\section{Conclusion}

Research on figure-ground organization has also come a long way since Wertheimer's (1923) paper on the Gestalt laws. First, when studied in more controlled experiments, the classic configural principles turn out weaker than previously supposed. Second, using richer displays, new figure-ground principles have been discovered with a plausible ecological foundation. Third, the role of past experience and attention has now been clearly demonstrated in experiments that satisfy Wertheimer's own criteria. Finally, figure-ground organization is no longer studied in isolation but turns out to be intimately related to other processes such as shape and depth perception. These connections are currently also the focus of interesting research on the neural mechanisms of contour grouping and figure-ground organization, as reviewed next.

\section{Neural Mechanisms in Contour Grouping, Figure- Ground Organization, and Border-Ownership Assignment}

\section{Introduction}

Recall that the failure of the experiments by Lashley et al. (1951) and Sperry et al. (1955) to support key predictions of Köhler's electromagnetic field theory of brain function was a devastating blow to Gestalt theory's attempt towards a radically different understanding of the brain mechanisms that underlie perception and cognition. The dominant model of brain function since then has been that the brain consists of an architecture of organized, interconnected neurons that interact via synaptic communication to form complex circuits that respond selectively to different properties of visual stimulation. Building on Hubel and Wiesel (1968)'s discovery and characterization of four types of neurons in the primary visual cortex (concentric, simple, complex and end-stopped), and the large number of neurophysiological studies that followed since then, the current view of the visual system is that of a hierarchy of stages of gradually increasing scale and complexity of processing, paralleled by the increasing receptive field size and increasing selectivity of neurons across the sequence of cortical areas in the ventral stream (see Rust \& DiCarlo, 2010, for a quantitative study).

It may seem that such a conception is fundamentally incompatible with Gestalt notions of brain function, but this is untrue. First, there is some evidence against such a strict hierarchical organization. For instance, measurements of receptive field size tend to underestimate the influence of context in neurons at the low levels, as many studies of so-called nonclassic surround influences have shown (Allman, Miezin, \& McGuinness, 1985; for further references, see Alexander \& van Leeuwen, 2010; Angelucci et al., 2002). Second, and more fundamentally, there is a more abstract level at which a Gestalt conception of brain function might be correct even if Köhler's conjecture about its implementation in dynamically converging electromagnetic brain fields is not. It is now clear that recurrent networks of neuron-like elementsnetworks that contain closed feedback loops-are a much more plausible implementation of the hypothesis that the brain is a physical Gestalt. Physicist John Hopfield (1982) clarified this possibility when he proved that symmetric recurrent networksnetworks with equal weightings in both directions between any pair of units-will always converge to an equilibrium state that satisfies an informational constraint isomorphic to minimum energy in physics. Hence, the Gestaltists may have been wrong in detail about the brain being an electromagnetic Gestalt system, but they may still have been correct at a more abstract level if recurrent networks are found to be crucial for perceptual organization. Important models of organizational phenomena within recurrent networks have indeed been proposed by Grossberg and others (e.g., Grossberg \& Mingolla, 1985; Kienker et al., 1986). Furthermore, while neurophysiological models for contour integration based upon good continuation principles have been based primarily upon cortical networks in area V1 (Li, 1998; Roelfsema, 2006; Yen \& Finkel, 1998), as well as recurrent interactions between areas V1 and V2 (Neumann \& Sepp, 1999), functional magnetic resonance imaging (fMRI) studies of contour grouping in both human and macaque implicate not only V1 and V2 but other 
extrastriate visual areas such as V4 and LOC (e.g., Altmann, Bülthoff, \& Kourtzi, 2003; Kourtzi, Tolias, Altmann, Augath, \& Logothetis, 2003).

Rather than providing a list of putative neural correlates of all the Gestalt principles, this section reviews neurophysiological studies investigating the neural mechanisms in contour grouping, figure-ground organization, and border-ownership assignment in an integrated way. In doing so, we demonstrate how contemporary neuroscience has embraced Gestalt ideas, while doing justice to Hubel and Wiesel's heritage in the following three ways: (a) We demonstrate how the responses of cortical neurons can depend on the parameters of the stimulus in its receptive field as well as on the properties of the overall configuration in the visual field, (b) we substantiate the Gestalt postulate of autonomous organization processes that form primary units of perception, and (c) we refine our understanding of the role of attention in these processes of perceptual organization.

\section{Context Integration in Illusory Contours}

Illusory contours were among the earliest demonstrations of perceptual organization (Schumann, 1900). Gestalt theory explains these contours by completion processes in the visual cortex. Initially, Sillito and colleagues (discussed in Gregory, 1987) recorded single neuron responses in cat primary visual cortex and found no evidence for illusory contour signals. Neurons that clearly responded to low-contrast figures were silent when illusory figures with the same perceived contrast were presented. However, recording from an area one level higher, namely, in V2 of the monkey visual cortex, von der Heydt, Peterhans, and Baumgartner (1984) found illusory contour responses in about one third of the recorded neurons. The stereotyped nature of the responses to repeated stimulation and their short latency indicated that the observed responses were stimulus driven rather than resulting from higher level, cognitive predictions as hypothesized by Gregory (1972).

Coren (1972) noticed that illusory contours generally arise in situations that suggest occlusion. One of the many models that have been proposed to explain illusory contours proposes that the V2 responses reflect a general mechanism for the detection of occluding contours (Heitger, von der Heydt, Peterhans, Rosentha- ler, \& Kübler, 1998; Peterhans \& von der Heydt, 1989). Under natural conditions, objects often occlude one another, and detecting the borders between image regions corresponding to foreground and background objects is a basic task of vision. V2 mechanisms might combine two sources of evidence for occluding contours: the presence of a luminance/color edge and the presence of occlusion features along the contour. Luminance/color differences can be detected by simple and complex cells and occlusion features (i.e., terminations of background structures) by endstopped cells (Heitger, Rosenthaler, von der Heydt, Peterhans, \& Kübler, 1992). By integrating inputs from simple/complex cells and appropriately selected end-stopped cells, V2 neurons can signal occluding contours more reliably than mechanisms based on edge detection alone (Heitger et al., 1998).

This model can be considered as a simple implementation of the Gestalt completion principle according to which illusory contours are formed in situations in which unbalanced shapes tend to completion (Kanizsa, 1979; Michotte et al., 1964). For example, the contours of an illusory white square in a configuration with four black pacmen are thought to emerge because the disc sectors tend to complete to circular discs (see Figure 22A). In the model, the terminations of the circular edges activate end-stopped cells. This mechanism allows for the possibility that the terminations are caused by occlusion and that the edges might continue in the background. While the principle of combining edge evidence with evidence from occlusion features for the definition of contours is valid, the model might be too simplistic because it fails to account for illusory contour perception in stereoscopic displays (Gillam \& Nakayama, 2002). Also, models of illusory contours in Kanizsa figures should be able to explain why the illusion disappears in other configurations (e.g., four crosses instead of four pacmen; see Figure 22B), which is a clear indication of the important role of the whole stimulus configuration, in line with the basic tenets of Gestalt psychology (see Kogo, Strecha, Van Gool, \& Wagemans, 2010, discussed further in the next section).

\section{Figure-Ground Organization and Border-Ownership Assignment}

The mechanism of illusory contours has obvious spatial limits: Illusory contours disappear when gaps become larger than a few
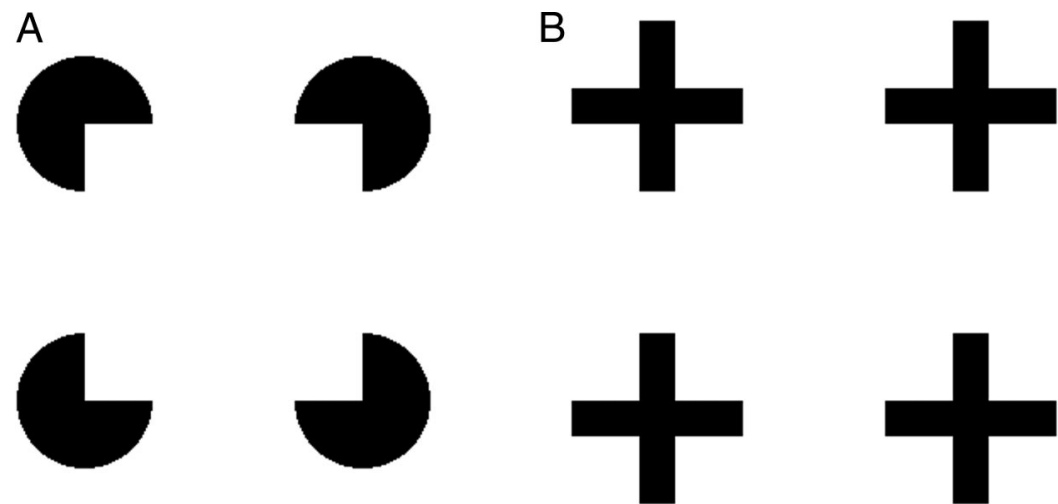

Figure 22. In a configuration with four black pacmen, an illusory white square emerges in the center, which does not happen when the same local edges occur in a configuration with four black crosses. 
degrees of visual angle (Peterhans \& von der Heydt, 1989). However, the spatial range at which figure-ground organization occurs is impressive, given the small receptive fields in the visual cortex. Even the extent of the nonclassic surrounds, which is typically about twice the size of the classic receptive field (see Cavanaugh, Bair, \& Movshon, 2002; Levitt \& Lund, 2002), is insufficient to explain the spatial range of figure-ground organization. It was therefore a surprise when Lamme (1995) discovered that neurons in monkey V1 show enhanced responses in figure regions compared to ground regions (see also T. S. Lee, Mumford, Romero, \& Lamme, 1998; Zipser, Lamme, \& Schiller, 1996). The figures in these studies were defined by a difference in texture, which could be based on orientation, motion, depth, or color, suggesting a general mechanism. Although the figure-ground modulation decreased with figure size and disappeared for figures larger than $8^{\circ}$, the range of context integration was clearly much larger than the typical size of the receptive fields (but see Rossi, Desimone, \& Ungerleider, 2001).

While Lamme's (1995) finding suggests coding in terms of regions, perceptual studies also emphasize the distinctive role of the borders between regions. Bregman (1981) has published a striking demonstration of this: Figure 23a shows a number of apparently meaningless shapes, while Figure $23 \mathrm{~b}$ shows exactly the same shape fragments, but with a blotch of ink present which is removed in Figure 23a. Because the blotch is seen as a foreground object, it takes ownership of some of the borders of the original shapes, which now organize to letters due to amodal completion of the shape fragments behind the blotch. The crucial role of border ownership in image interpretation was rediscovered by Nakayama, Shimojo, and Silverman (1989), and it was also recognized in computational modeling studies (Finkel \& Sajda, 1992), but how border ownership might be coded by neurons was less clear until recordings from monkey visual cortex by $\mathrm{H}$. Zhou, Friedman, and von der Heydt (2000) revealed that the firing rate of orientation-selective neurons that respond to the contours of figures depended on where the figure is located relative to the receptive field of the neurons. Moreover, each neuron was found to have a fixed preference for direction of figure, while across neurons all directions in the visual field were represented equally. As a consequence, each border is represented by two groups of neurons, one for each side of ownership. Zhou et al. suggested that the differential activity between the two represents the borderownership assignment. In area V2, about half of the oriented neurons are border-ownership selective. Such neurons can also be found in V1 and V4, but less frequently.

Two characteristics of border-ownership coding are remarkable: the large range of context integration and the short latency of the differential response. The context influence extends far beyond the classic receptive field, and it appears less than $30 \mathrm{~ms}$ after the earliest responses in V1 or V2 (60-70 ms after stimulus onset), which is earlier than the figure-ground enhancement in V1 (Lamme, 1995). Tests with fragmented figures showed that most or all segments of the figure contours contribute (Zhang \& von der Heydt, 2010). These findings have strong implications for possible neural mechanisms, in particular for the role of horizontal interactions, which is often postulated in models of figure-ground organization (e.g., Finkel \& Sajda, 1992; Grossberg, 1994; Kogo et al., 2010; Sajda \& Finkel, 1995; Zhaoping, 2005). Close examination of the conduction delays shows that the horizontal propagation scheme is incompatible with the large range of context integration and the short latency of the context influence (Zhang \& von der Heydt, 2010; for a discussion, see Craft, Schütze, Niebur, $\&$ von der Heydt, 2007). There is little evidence for propagation delays in context integration for border ownership. Instead, Zhang and von der Heydt (2010) found that the influence of the most distant contour segments arrived earlier than that of the segments closest to the receptive field. Hence, it is more likely that context integration involves feedback from higher level visual areas (e.g., Craft et al., 2007; Jehee, Lamme, \& Roelfsema, 2007; Roelfsema, Lamme, Spekreijse, \& Bosch, 2002): Neurons in these areas have larger receptive fields and signals between areas travel through white matter fibers, which are much faster than the intracortical horizontal fibers. These models are in agreement with studies

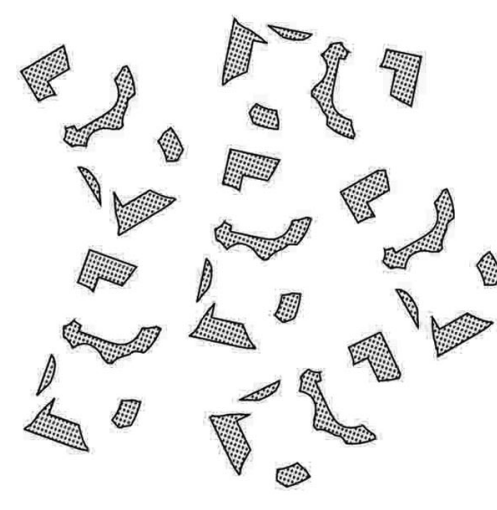

A

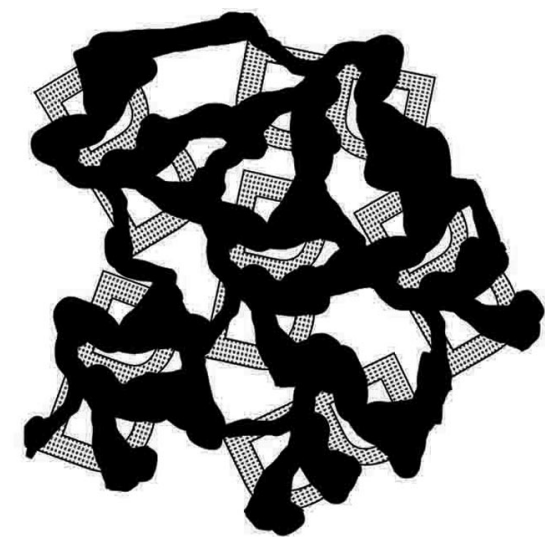

B

Figure 23. A: A quasi-random collection of quasi-random shapes. B: The same shapes as in Panel a with a black ink blotch, which is seen to occlude five letters B. Adapted from "Asking the 'What for' Question in Auditory Perception,” by A. S. Bregman, in Perceptual Organization (pp. 106-107), ed. by M. Kubovy and J. R. Pomerantz, 1981, Hillsdale, NJ: Erlbaum. Copyright 1981 by Lawrence Erlbaum Associates. Adapted with permission. 
showing that the far surround of receptive fields in V1 is contributed by feedback (Angelucci et al., 2002).

The involvement of border ownership and feedback in figureground organization have much to say about its relevance in everyday vision. Figure-ground organization is obviously related to the task of interpreting 2-D images in terms of a 3-D world, which is fundamental to vision. What Gestalt psychology has revealed is the compulsion of the system to seek a 3-D interpretation, even under the impoverished conditions of simple drawings that do not provide information about depth. When explicit depth information is available, for example, in stereoscopic vision, it has a profound influence on image segmentation and perceptual organization (Gregory \& Harris, 1974; Nakayama et al., 1989; Shimojo, Silverman, \& Nakayama, 1989). The influence of stereoscopic depth is an important criterion for the validity of the interpretation of the neural signals. If response enhancement and border-ownership modulation indeed participate in the attempt to fit a 3-D interpretation, then these signals should respond to border ownership given by disparity as well as by 2-D cues, but if they reflect general receptive field mechanisms (e.g., center-surround antagonism), there would be no reason for this parallelism. Several neurophysiological studies have shown that stereoscopic depth can indeed dramatically influence the cortical responses to a visual stimulus, even in early visual regions (Bakin, Nakayama, \& Gilbert, 2000; Duncan, Albright, \& Stoner, 2000; Qiu \& von der Heydt, 2005; Zipser et al., 1996).

For instance, applying Lamme's figure-ground paradigm to random-dot stereograms, Zipser et al. (1996) showed that the enhancement is not simply the result of surroundedness, but depends on the conditions of figure perception. When a surface in the fixation plane (zero disparity) is surrounded by a region of far disparity, it assumes figure status and produces enhancement of responses; it appears as an object floating in front of a background. In contrast, when it is surrounded by near disparity, it assumes ground status and no enhancement occurs; the surrounded region now appears as a surface in the back that is seen through a window. Stereoscopic depth also has a strong influence on the coding of border ownership. Qiu and von der Heydt (2005) found that many V2 cells combines border-ownership selectivity (for contrastdefined figures without disparity) with stereo-edge selectivity in random-dot stereograms, and the preferred side of the figure was generally the same as the preferred foreground side in the stereograms. This means that the cortex interprets a contrast-defined figure as an object occluding a background, in line with the Gestalt view.

The above findings have recently been integrated in a neurocomputational model (Kogo et al., 2010) in which border ownership and occlusion play a central role in the emergence of illusory surfaces and contours in Kanizsa-type displays (see Figure 22 again). Specifically, local occlusion cues ( $\mathrm{L}$ and $\mathrm{T}$ junctions) trigger a perceived depth difference and border-ownership signals at the visible edges, which then spread and combine with borderownership signals in the open space between the pacmen. This spreading and enhancement of border ownership only occurs with particular global configurations of the visible elements (the pacmen) that are consistent with a central surface (e.g., not in the four-crosses configuration). In contrast with many previous models, this model is not aimed at contour completion based on the collinearity of the local image fragments. Instead, it achieves surface completion triggered by local depth cues that are consistent with a particular global configuration, which is more in line with the original Gestalt view of the phenomenon. Moreover, it treats the neural responses by V1 cells not as feature detectors but as 2-D differentiated signals. These signals are then spatially integrated in 2-D surfaces, allowing a reinterpretation of well-known facts from single-cell recordings into a more global, functional, perceptual framework in the Gestalt tradition.

\section{Involuntary Organization and Volitional Attention}

The classic Gestalt psychologists demonstrated the existence of an early, autonomous process of visual organization, producing percepts that do not always conform to previous knowledge or expectations about the stimulus. Small changes to the stimulus can induce extensive perceptual reorganization. For example, a display consisting of two light and two dark squares on a medium gray background (see Figure 24b) can easily be turned into a display that looks like two crossed bars, one light and one dark, in transparent overlay (see Figure 24c), when the corners are made sharp instead of rounded. Recordings of border-ownership signals in visual cortex demonstrate how a stimulus is interpreted and reorganized in this case (Qiu \& von der Heydt, 2007). When the rounded squares were presented, the border-ownership signals at the edges pointed to the inner side of the squares, but when the straight squares were presented, the signals pointed to the inside of what was perceived as bars. The signals on the edges bounding the region of apparent overlap had now reversed. Hence, a slight modification of the conditions of good continuation leads to a spontaneous reorganization of shapes, which can also be observed in area V2 of the visual cortex (see Figure 24).
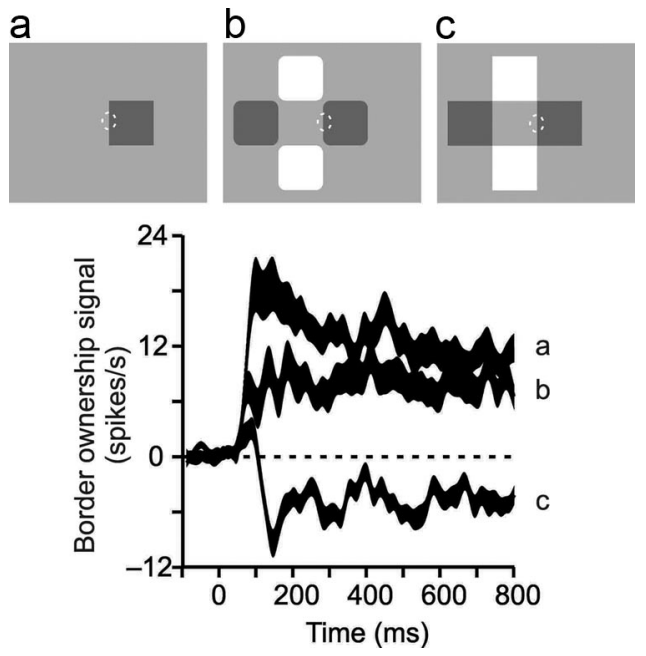

Figure 24. The isolated square (Panel a) and the squares with rounded corners (Panel b) appear as distinct objects, but when the squares come in contact at the corners, two bars in transparent overlay are perceived (Panel c). The small alteration of contours results in a perceptual reorganization. Note the reversal of border ownership at the marked edge. The population border ownership signal in the visual cortex (area V2) also shows this reversal (curves below). The dashed ellipses mark the receptive field positions. 
Such reinterpretations do not seem to require a shift of attention, but the role of attention in perceptual (re)organization is not a trivial matter. Gestalt psychologists have pointed out that attention is drawn to figures, whereas the background regions often go unnoticed. How unattended objects are processed is difficult to derive from phenomenal reports by participants who cannot avoid paying attention to the stimuli that they are supposed to judge. In contrast, neuronal recordings show the processing of all stimuli, whether attended or not. Neurophysiological studies investigating whether perceptual organization processes are preattentive, are influenced by attention, or take place only under attention have produced mixed results. In some situations, attention initiates a process of organization that reflects the intrinsic connectivity of the cortex. In other situations, organization emerges independently of attention, creating a structure for selective attention. For example, when monkeys were trained to mentally trace curves from the fixation point to a target, V1 neurons with receptive fields on the curves showed enhanced responses when the monkey traced the curve that passed through the receptive field, compared to when it traced the other curve (Roelfsema, Lamme, \& Spekreijse, 1998). The performance of the tracing task is thought to depend on collinear facilitation between neurons with neighboring receptive fields of similar orientation (Gilbert, 1992), gated by attention (Ito \& Gilbert, 1999), resulting in a spread of enhancement from the fixation point along the curve (Roelfsema, Lamme, \& Spekreijse, 2000; Roelfsema, Scholte, \& Spekreijse, 1999). More recent experiments have established similar effects for other grouping principles as well (Wannig, Stanisor, \& Roelfsema, 2011).

From these experiments it might seem that binding and grouping depend on attention, much as proposed by Treisman and Gelade (1980), but with the difference that the structure develops according the connectivity in the cortex (specifically V1). However, studies on border-ownership selectivity show that there is also preattentive organization (Qiu, Sugihara, \& von der Heydt, 2007). For example, when monkeys performed a task that required selective attention to one of several figures in a display, it was found that the activity of most neurons was modulated independently by border ownership and by attention. Moreover, the borderownership signals in these neurons emerged simultaneously at attended and unattended figures. This shows that border-ownership signals are generated in parallel for the various objects in the display without the involvement of attention.

Although border-ownership signals can be generated without active allocation of attention, attention can also exert an influence on border assignment. This was demonstrated in an experiment by Qiu et al. (2007), who presented two overlapping figures and measured the neuronal response to the border between the figures (the occluding contour), varying border ownership and the side of attention. They found that attention could enhance or reduce the border-ownership signal produced by the overlap. When the occluding figure was attended, the population border-ownership signal for the occluding contour was enhanced, but when the occluded figure was attended, the signal was abolished (extrinsic edge suppression; cf. Nakayama et al., 1989). For the border between two abutting figures (where border ownership is ambiguous), the signal would be determined by the side of attention. This corresponds to perception in Rubin's ambiguous vase figure, where border ownership can be flipped deliberately by changing attention from the vase region to a face region and vice versa (although spontaneous, automatic switches occur as well).

The interactions between attention and border ownership may seem complicated, but there is an additional observation that suggests that the underlying mechanism is simple: The side of attentive enhancement and the preferred side of border ownership of a neuron tend to be the same (Qiu et al., 2007). This indicates that the same neural circuits that make a neuron border-ownership selective also produce the modulation by attention. One can explain all the above results by assuming that the edge signals produced by a figure are summed by a common grouping cell that, by feedback, sets the gain of the corresponding edge neurons (Craft et al., 2007). Each border-ownership selective V2 neuron is connected to grouping cells on one side of its receptive field, and therefore shows enhanced responses when a figure is present on that side. The effects of attention are explained by assuming that volitional attention activates the grouping cells corresponding to the object to be attended (Mihalas, Dong, von der Heydt, \& Niebur, 2011). This raises the gain of the connected edge neurons. The one-sided connectivity of the edge neurons accounts for the asymmetric attention effect, and, because the same grouping cells produce border ownership and attentive modulation, the side of attentive enhancement is also the preferred side of border ownership.

\section{Conclusion}

In sum, the neurophysiological evidence from the last 2 decades seems to converge on the idea that the responses of cortical neurons depend on the properties of the overall configuration in the visual field as well as on the parameters of the stimulus in its receptive field. The connectivity and rules of the visual cortex allow illusory contours to be formed and figure-ground segmentation to be performed by autonomous processes that are at the same time also context sensitive. The segregation of automatic and volitional processing closely resembles what the pioneers of $\mathrm{Ge}$ stalt psychology envisioned, and many of the details currently discovered show parallels to the perceptual phenomena that they pointed out. As indicated by Westheimer (1999), neurophysiology has come a long way since Hubel and Wiesel's (1968) atomistic approach to orientation selectivity of single cells in cat and monkey cortex, which were taken as prototypical feature detectors. The literature reviewed above matches well with the premature physiological theory postulated by Wertheimer (1922/1938a, p. 15): "The cells of an organism are parts of the whole and excitations occurring in them are thus to be viewed as part-processes functionally related to whole-processes of the entire organism." Indeed, it gives a concrete meaning to it, by emphasizing the role of context-sensitive, autonomous processes within recurrent networks.

\section{General Discussion and Conclusion}

One century of research on perceptual grouping and figureground organization has yielded a wealth of knowledge regarding principles of perceptual organization, their ecological foundations, computational mechanisms, and neural underpinnings. In addition, it allows us to reflect upon the waxing and waning of views on the relationships between elementary sensations and integrated per- 
cepts. In this final section, we evaluate what Gestalt psychology has offered, how its limitations were overcome, how many of its main ideas still affect contemporary thinking about visual perception, and what the remaining challenges are for future research on perceptual organization.

\section{The Swinging Pendulum of Gestalt History}

Based on the discovery of phi or pure motion (i.e., perceived motion without objects being perceived as moving) exactly 100 years ago, Wertheimer (1912) and his fellow-pioneers of the Berlin school of Gestalt psychology arrived at some far-reaching conclusions causing a true revolution in psychology, philosophy, and neighboring disciplines (see also Table 3 again). Demonstrating a case where the phenomenological experience was clearly not composed of more elementary sensations, they concluded that structured wholes or Gestalten, rather than sensations, must be the primary units of mental life. They argued that the contents of awareness were not produced from associations or combinations between sensations. The whole is not only more than the sum of the parts, it is different because it has whole-properties that determine the part-properties as much as the other way around (i.e., two-sided dependency between wholes and parts). From the very beginning, these Gestalts were assumed to arise on the basis of continuous whole-processes in the brain, involving the entire optical sector from retina to cortex.

To substantiate these revolutionary ideas, phenomenological analysis of visual demonstrations was used to discover the laws of perceptual organization governing the experienced Gestalts (Wertheimer, 1923). A plethora of interesting phenomena could be shown, but deriving laws from them proved to be harder: Even when the demonstrations were supplemented with parametric experiments, the stimuli were often very simple, and the ceteris paribus principles derived from them were easily destroyed by small extensions beyond the original constraints, yielding abundant exceptions to the rule. In somewhat richer stimuli, different factors determining the perceived organization interacted unpredictably, in line with the Gestalt spirit, but frustrating from the perspective of formulating laws.

To avoid a proliferation of laws, the law of Prägnanz was proposed as the fundamental law encompassing all the others but its formulation was left intentionally vague: "psychological organization will always be as 'good' as the prevailing conditions allow" (Koffka, 1935, p. 110), and "on the whole the reader should find no difficulty in seeing what is meant here. ... One recognizes a resultant 'good Gestalt' simply by its own 'inner necessity", (Wertheimer, 1923/1938c, p. 83). Inspiration was sought in physical phenomena that appeared to show similar global effects, in order to formulate field models of electric currents in the brain, which were supposed to be structurally and functionally isomorphic to the experienced Gestalts (Köhler, 1920). But when Köhler's electrical field theory lost its empirical basis due to Lashley's and Sperry's experiments, no alternatives were found to replace the physical but nonmechanistic foundations of Gestalt theory. With no testable quantitative models and no plausible neural underpinning, the Gestalt principles remained mere descriptions of interesting perceptual phenomena.

The discovery of single neurons being tuned to primitive stimulus attributes (e.g., line orientation, motion direction) in the 1950 s led to a predominantly atomistic approach in neuroscience, and around the same time, computers models appeared to provide testable, mechanistic accounts of mental operations. Although the Gestalt line of work continued in relatively isolated corners of science (e.g., Metelli and Kanizsa in Italy, Michotte in Belgium, Oyama in Japan), the mainstream around that time was very much non-Gestaltist, if not anti-Gestaltist.

This started to change again when new Gestalt-like phenomena were discovered such as global precedence and configural superiority effects (e.g., Navon, 1977; Pomerantz et al., 1977). Perceptual organization became fashionable again (e.g., J. Beck, 1982; Kubovy \& Pomerantz, 1981), partly because the Gestalt principles were thought to deliver suitable computational constraints on computer vision algorithms (e.g., Marr, 1982), and partly because neurophysiological studies revealed contextual modulation effects on cell responses from outside the cell's classic receptive field (e.g., Allman et al., 1985) and clear neural correlates of Gestalt phenomena such as illusory contours (e.g., von der Heydt et al., 1984). As a result, the last 2 or 3 decades have seen a significant resurrection of fruitful empirical work on perceptual organization, to the extent that one could speak of a Gestalt revival, not only in the domain of visual perception (e.g., recent reviews on Gestalt principles in tactile perception by Gallace \& Spence, 2011, and in motor action by Klapp \& Jagacinski, 2011).

\section{Gestalt Research Anno 2012}

Compared to the troublesome situation of Gestalt research in the mid-20th century, contemporary vision science has made a lot of progress regarding perceptual grouping and figure-ground organization (see Table 4, right column again). Phenomenological demonstrations with either very simple or confounded stimuli were supplemented with real experiments, using carefully constructed stimuli (e.g., dot lattices, Gabor displays) that allowed for parametric control, and sometimes also richer stimuli, in which cue combinations could take place. Isolated cues in well-controlled displays usually lead to weaker effects, whereas disparate weak cues often combine synergistically in more natural images. Exploiting the potential of modern techniques to create controlled but richer stimulus displays has also led to the discovery of new principles of grouping (e.g., generalized common fate, synchrony) and of figure-ground organization (e.g., extremal edges, articulating motion).

In addition to direct reports of perceptual experiences, indirect behavioral measures were developed, employing standard tools from experimental psychology (e.g., cuing, priming, matching) and psychophysics (e.g., response-bias free performance indices, thresholds). In most situations, these experiments enabled quantification of the strength of the factors influencing perceptual grouping and figure-ground organization, in some cases even formulated as real laws (e.g., pure distance law in grouping by proximity), or as clear demarcations between distinctive regimes (e.g., space-time coupling vs. tradeoff in apparent motion). In some areas of research, solid experimentation was accompanied by the development and testing of computational models, often starting from strong geometrical descriptions at the stimulus level or careful analysis of the natural image statistics, and then using the framework of statistical decision theory to characterize the regularities in the frequencies of responses. In other areas of research, 
psychophysical results could be related to neural mechanisms, at least in principle, by using similar stimuli and paradigms (e.g., illusory contours, figure-ground segmentation).

Hence, research on perceptual grouping and figure-ground organization has been able to build on the sophisticated research methods available in vision science in general, and this has also allowed for a somewhat less isolated treatment of these processes. Some progress has been made in linking these aspects of perceptual organization to other aspects of visual processing, to the extent that mid-level vision is often conceived as a relay station between low-level vision with its rather hardwired extraction of primitive stimulus attributes (e.g., contrast, spatial frequency, orientation, motion direction) and high-level vision processes that interpret their meaning. In computer vision, grouping principles have been used to facilitate image processing, and image regularities have been used to facilitate the recovery of 3-D shapes from 2-D images. Biederman (1987) incorporated much of this progress in image understanding in his theory of human object recognition, thereby integrating Gestalt principles into mainstream cognitive science.

As a result of these decades of research on Gestalt issues with more modern techniques and in light of more current views, more moderate, sometimes even synthetic positions are now taken with respect to several of the traditional controversies (see Table 5 for an overview). In other words, although the negative attitude towards Gestalt psychology is gradually disappearing, modern vision science cannot be considered some kind of contemporary Gestalt approach either. First, much of what has been discovered goes against Gestalt psychology, as originally conceived. Rather than being primary, in the sense of preattentive and early, principles of grouping seem to operate at multiple levels, and although figureground segregation can occur preattentively, it can also be affected by attention. Whereas old-school Gestalt psychology emphasized that Gestalt laws are innate and intrinsic rather than learned from experience, recent studies with adult observers showed that past experience can exert an influence on several aspects of figureground perception. Moreover, although infants are already capable of grouping according to at least some grouping principles, developmental trends regarding other grouping principles indicate that visual experience does play a role as well. More generally, the convergence between psychophysical results and natural image statistics seems to indicate that the visual system is tuned to the properties of its environment. This seems to increase the validity of approaches emphasizing the importance of veridicality of perception and a general likelihood principle. In any case, within the Gestalt tradition, it raises the question of how internal laws based on a general minimum or simplicity principle might yield veridicality in the external world. (This issue is taken up again in the second article; see Wagemans et al., 2012, Sections 5 and 6.)

Second, modern vision science appears to be incommensurate with "the fundamental 'formula' of Gestalt theory" (Wertheimer, 1924/1938b, p. 2): "There are wholes, the behavior of which is not determined by that of their individual elements, but where the part-processes are themselves determined by the intrinsic nature of the whole." Like the Gestaltists' rivals in the early days, much of contemporary science is analytic rather than holistic. Even when configural effects are shown to occur time and time again, and even when strictly bottom-up models are replaced by more realistic models emphasizing feedforward-feedback loops and reentrant processing (e.g., Hochstein \& Ahissar, 2002; Jehee et al., 2007; Lamme, 1995; Roelfsema, 2006; Roelfsema et al., 2002), this is still a far cry from recognizing the primary nature of structured wholes in experience, the importance of two-sided dependency between parts and wholes, and global field dynamics. In fact, Gestalt phenomena are still not very well integrated into mainstream thinking about the visual system's operating principles (e.g., selectivity and tuning of single cells, V1 as a bank of filters or channels, segregated what and where streams, increasing receptive field size and invariance at higher levels of the hierarchy, specialized modules for separate object categories such as faces

Table 5

Current More Synthetic Positions

Some Gestalten are strong Gestalten, others are weak Gestalten.

Some Gestalten arise suddenly and as immediately organized wholes, but sometimes it is useful to examine gradual emergence or microgenesis.

Grouping principles operate at multiple levels: Grouping occurs both pre- and postconstancy.

Grouping principles sometimes combine additively, sometimes nonadditively.

Historically, Gestalt psychology has emphasized the degree to which the Gestalt laws are innate or intrinsic to the brain rather than learned from past experience, but there is now also a lot of attention to the development of perceptual organization and to the role of past experience. For instance, we now know that not all grouping cues are readily available to young infants and that there is a protracted developmental trajectory for some perceptual organization abilities, even those that appear to emerge during infancy.

How the brain combines multiple cues to yield a unitary organization has often been posed in terms of competitive interactions formulated either in descriptive terms (usually seeking compliance with the simplicity principle) or in probabilistic terms (mostly Bayesian formulations which may or may not seek compliance with the Helmholtzian likelihood principle). In natural scenes, however, disparate weak cues can often combine synergistically to yield strong evidence for a particular grouping.

Figure-ground organization is driven by image-based cues as well as by subjective factors such as past experience (familiarity), attention, and perceptual set.

Figure-ground organization does not always occur early in the visual system; it can be affected by focused attention, but it can also occur preattentively.

Neurophysiological studies investigating whether perceptual organization processes are preattentive, are influenced by attention, or take place only under attention have produced mixed results. In some situations, attention initiates a process of organization that reflects the intrinsic connectivity of the cortex. In other situations, organization emerges independently of attention, creating a structure for selective attention.

The neurophysiological evidence from the last 2 decades seems to converge on the idea that the responses of cortical neurons depend on the properties of the overall configuration in the visual field as well as on the parameters of the stimulus in its receptive field. The connectivity and rules of the visual cortex allow illusory contours to be formed and figure-ground segmentation to be performed by autonomous processes that are at the same time also context sensitive. 
and places). Or, formulated more positively, establishing such an integration continues to provide serious challenges.

In sum, the current focus on Gestalt issues has not given rise to a new coherent school of thought, as it existed in the first few decades of Gestalt research. Current research on perceptual grouping and figure-ground organization is integrated well within mainstream vision science mainly regarding the research methods and techniques, not as much regarding its results, and certainly not regarding its deeper implications. It does not itself form an integrated domain of research, let alone a coherent research program or theoretical framework guided by a limited set of (meta)theoretical principles as their foundations, as was the case in the Berlin school of Gestalt psychology, started by Wertheimer (1912) a century ago. In addition to this major (meta)theoretical challenge, some other limitations of contemporary research on perceptual organization should be pointed out as well (for an overview, see Table 6).

\section{Limitations and Challenges to Contemporary Research on Perceptual Organization}

Perceptual grouping and figure-ground organization, although intimately connected, are not the same process. Perceptual grouping is concerned with the binding together of elements that are disjointed at the level of the proximal stimulus (retinal images). Often, but not always, grouping also entails its complementleaving out some elements as noise or background elements, not selected for further processing. However, this does not mean that the group of selected and grouped elements gets figural status and that the nonselected, nongrouped elements become a background that continues behind the first group. This seems to require special conditions: "Phenomenal figures have boundary lines even when the corresponding objective figures have none. A good figure is always a 'closed' figure, which the boundary line has the function of closing" (Koffka, 1922, p. 14). Hence, it is clear that groups do not necessarily obey the same Gestalt properties as figures, and that grouping does not necessarily behave according to the same principles as figure-ground assignment. It would be interesting to focus more on the similarities and differences between the properties of groups and figures, and to characterize them better, for instance, on a graded continuum from weak to strong Gestalts, depending on the mutual relationships between the parts and the wholes or on how linear or nonlinear their underlying processes are. There is some overlap in the list of factors determining grouping and figure-ground assignment, but others apply to only one of the two forms of organization. There is a clear need for a systematic analysis of the common factors (and whether they are common because they affect the same component process, or because the same factor just happens to influence two independent processes in the same way), as well as of the organization-specific factors (and whether this specificity is due to a major functional difference or is merely a side effect of task demands).

In general, a thorough examination of the specific task requirements induced by the stimulus and imposed by the instructions is needed to be able to determine the processes involved and the potential generalization beyond the test conditions. For instance, in research aimed at the quantification of grouping by proximity, dot lattices are used and observers are asked to indicate in which orientation they see the linear arrangements of dots. Stimuli are highly regular, percepts are multistable (near equilibrium), and phenomenal reports are asked. Grouping involves all elements here, and the selection is at the level of percepts. When one orientation is seen, the others are still present in the stimulus. It is probably the noise in the visual system (i.e., internal noise) that causes switching from one percept to another. The situation is quite different in research aimed at the quantification of good continuation, in which random arrays of Gabor elements are mostly used, and observers are asked to detect or locate the target group (snake) embedded in a background of noise elements. Here, noise is present in the stimulus (i.e., external noise), and target elements must be selected for proper grouping. A participant's response can be regarded as correct or incorrect, relative to the intended target group, although it is always possible that an observer truly sees a (spurious) group in the background elements, leading to a false alarm or mislocalization. How can we expect grouping principles to generalize between two such fundamentally different situations? Further progress with respect to theoretical integration will depend on experiments that bridge the gaps between different experimental paradigms, starting from analyses such as the above.

A similar recommendation applies to the connection between contour grouping, contour integration, contour completion, and the like. Stimulus and task differences complicate a theoretical synthesis. A major limitation of these studies is that they usually do not deal with contours in the sense of boundary lines with the

Table 6

Limitations and Challenges to Contemporary Research on Perceptual Organization

There is a clear need for a systematic analysis of the factors that are common to both perceptual grouping and figure-ground organization, and of the factors that are specific to one of them.

A thorough examination of the specific task requirements induced by the stimulus and imposed by the instructions is needed to be able to determine the processes involved and the potential generalization beyond the test conditions.

Further progress with respect to theoretical integration between different processes of perceptual organization will depend on experiments that bridge the gaps between different experimental paradigms.

Progress regarding figure-ground organization could profit from a more fine-grained analysis of the different components involved.

Linking experiments are needed to facilitate an integration of grouping and segregation processes into the figure-ground organization literature.

A fundamental limitation of current research on perceptual grouping as well as figure-ground organization is the shortage of computational process models.

Progress in developing and testing neurocomputational models, which are supposed to rely on solid computational principles that are compatible with known neurophysiology and human psychophysics, requires painstaking bridging efforts by multidisciplinary teams (e.g., psychophysicists, modelers, neurophysiologists, and neuroanatomists). 
function of closing an area or region. A snake in a Gabor array is a curvilinear group, it is not a contour, nor is it a boundary of a figure or an object (although the name itself refers to some object, accidentally). The literature on perceptual grouping in the context of interrupted or noisy contours in real images would be much more directly relevant to figure-ground organization if their snake stimuli were to be supplemented with curved groups that have more potential as boundaries of surfaces. Such research has been started (e.g., Machilsen et al., 2009; Machilsen \& Wagemans, 2011; Nygård, Sassi, \& Wagemans, 2011; Nygård, Van Looy, \& Wagemans, 2009; Sassi, Vancleef, Machilsen, Panis, \& Wagemans, 2010), but more is needed.

The same holds true in the other direction as well: Figureground organization could be related more strongly to perceptual grouping. Progress regarding figure-ground organization could profit from a more fine-grained analysis of the different components involved, from segregating regions (based on the relative similarity within a region/group and dissimilarity between different regions/groups), representing the contour with all of its relevant geometric properties (including grouping the contour fragments or linking the multiple borderline signals at different locations in the visual field), and relating these to relevant properties of the configuration within which the contour is embedded, to figure-ground assignment including the integration of multiple border-ownership signals and the overall border-ownership assignment. Moreover, such an interplay must be embedded into a dynamic system with cooperative and competitive units, with its own proper balance between deterministic and stochastic characteristics, to allow for perceptual switching to occur in cases of multistability. Again, crucial linking experiments are needed to facilitate such an integration of grouping and segregation processes into the figure-ground organization literature.

A fundamental limitation of current research on perceptual grouping as well as figure-ground organization is the shortage of computational process models. As mentioned earlier, most of the present models are statistical, descriptive models. In low-level vision, computational models exist that take stimulus images as input and produce binary task-related responses as output (e.g., yes/no in a detection task), spelling out all of the intermediate processing steps by equations with a limited set of free parameters. Such models can be fitted directly to psychophysical results obtained with human observers, usually yielding increased insight in the underlying mechanisms, or at least providing some constraints on fundamental principles that allow further progress to be made by refinement of the models or additional psychophysical testing. Although psychophysical methods have been used to directly compare thresholds for detecting deviations from uniformity and thresholds for grouping based on the same nonuniformities (Gori \& Spillmann, 2010), full-fledged computational models with integrated psychophysics are rare in mid-level vision (e.g., Geisler \& Super, 2000). One advantage that low-level vision has compared to mid-level vision is that it can build more directly on a strong and solid psychophysical tradition, in which stimuli, tasks, data analysis techniques, and computational models are more strongly integrated with one another, reflecting a more mature status of science. Significant steps forward could be taken if this quantitative computational tradition would be pushed forward from low- to mid-level vision.
Low-level vision can also relate more directly to neurophysiological findings than mid-level vision. Although we believe in the value of a thorough phenomenological analysis of interesting perceptual phenomena (e.g., Sinico, 2008), we are also convinced that explanations at a neural level provide considerable added value (e.g., Spillmann, 2009). Single-cell responses of V1 are very well characterized in relation to well-controlled low-level stimulus characteristics, and their response properties and tuning are relatively well understood (for a review, see Carandini et al., 2005). Where context effects or more realistic configurations are concerned, the combinatorial space of stimulus attributes becomes prohibitive for similar parametric studies (but see Brincat \& Connor, 2004, 2006, for a most interesting attempt).When true Gestalt experiences are at stake, either fundamental limitations of the research methods (fMRI or EEG) or problems with cross-species comparisons (from monkey to human) are unavoidable. As a result, success stories regarding direct neural correlates of interesting Gestalt phenomena are relatively rare. Similar progress regarding neurocomputational models, which are supposed to rely on solid computational principles that are compatible with known neurophysiology and human psychophysics, requires painstaking bridging efforts and also raises many open questions with respect to specific details that can be addressed only by multidisciplinary teams (e.g., psychophysicists, modelers, neurophysiologists, and neuroanatomists).

Perceptual grouping and figure-ground organization are two important aspects of perceptual organization, but they are not the only ones. Also interesting are, for instance, the representation of part-whole relationships in a hierarchical structure, texture segregation, geometric distortions due to context or field effects, embedded figures, and holistic processing of faces. Many of the above lessons we derived from this review also apply to these other areas of mid-level vision.

\section{Conclusion}

We hope this review has demonstrated that rumors about the death of Gestalt psychology were greatly exaggerated (Epstein, 1988). On the contrary, the field of research on perceptual grouping and figure-ground organization is thriving, and progress has been tremendous compared to the situation of 100 years ago. However, significant challenges remain. The above discussion has outlined an open-ended research program, like Wertheimer and his fellow-pioneers did. However, we are now able to build on a research tradition of more than a century. We are convinced that we can reconsider some of the old puzzles at a much more advanced scientific level now. The most important challenge will be to integrate better this research tradition with the rest of vision science. Such an integration will strongly depend on progress regarding the conceptual and theoretical foundations of the Gestalt approach. This will be the focus of the second of this twin set of review articles on the occasion of the centennial anniversary of Gestalt psychology (Wagemans et al., 2012).

\section{References}

Alais, D., Blake, R., \& Lee, S. H. (1998). Visual features that vary together over time group together over space. Nature Neuroscience, 1, 160-164. doi: $10.1038 / 1151$ 
Alexander, D. M., \& van Leeuwen, C. (2010). Mapping of contextual modulation in the population response of primary visual cortex. Cognitive Neurodynamics, 4, 1-24. doi:10.1007/s11571-009-9098-9

Allman, J., Miezin, F., \& McGuinness, E. (1985). Stimulus-specific responses from beyond the classical receptive field: Neurophysiological mechanisms for local-global comparisons in visual neurons. Annual Review of Neuroscience, 8 , 407-430. doi:10.1146/annurev.ne.08.030185.002203

Altmann, C. F., Bülthoff, H. H., \& Kourtzi, Z. (2003). Perceptual organization of local elements into global shapes in the human visual cortex. Current Biology, 13, 342-349. doi:10.1016/S0960-9822(03)00052-6

Anderson, B. L. (2007). The demise of the identity hypothesis and the insufficiency and nonnecessity of contour relatability in predicting object interpolation: Comment on Kellman, Garrigan, and Shipley (2005). Psychological Review, 114, 470-487. doi:10.1037/0033-295X.114 .2 .470

Anderson, B. L., Singh, M., \& Fleming, R. W. (2002). The interpolation of object and surface structure. Cognitive Psychology, 44, 148-190. doi: 10.1006/cogp.2001.0765

Angelucci, A., Levitt, J. B., Walton, E. J. S., Hupé, J. M., Bullier, J., \& Lund, J. S. (2002). Circuits for local and global signal integration in primary visual cortex. Journal of Neuroscience, 22, 8633-8646.

Arnheim, R. (1967). Art and visual perception. Berkeley: University of California Press.

Ash, M. G. (1995). Gestalt psychology in German culture, 1890-1967: Holism and the quest for objectivity. Cambridge, England: Cambridge University Press.

Attneave, F. (1954). Some informational aspects of visual perception. Psychological Review, 61, 183-193. doi:10.1037/h0054663

Attneave, F. (1971, December). Multistability in perception. Scientific American, 225(6), 62-71. doi:10.1038/scientificamerican1271-62

Attneave, F., \& Arnoult, M. D. (1956). The quantitative study of shape and pattern perception. Psychological Bulletin, 53, 452-471. doi:10.1037/ h0044049

Bahnsen, P. (1928). Eine Untersuchung über Symmetrie und Asymmetrie bei visuellen Wahrnehmungen [An investigation of symmetry and asymmetry in visual perception]. Zeitschrift für Psychologie, 108, 129-154.

Bakin, J. S., Nakayama, K., \& Gilbert, C. D. (2000). Visual responses in monkey areas $\mathrm{V} 1$ and $\mathrm{V} 2$ to three-dimensional surface configurations. Journal of Neuroscience, 20, 8188-8198.

Barenholtz, E. (2010). Convexities move because they contain matter. Journal of Vision, 10(11), Article 19. doi:10.1167/10.11.19

Barenholtz, E., \& Feldman, J. (2006). Determination of visual figure and ground in dynamically deforming shapes. Cognition, 101, 530-544. doi:10.1016/j.cognition.2005.12.002

Barenholtz, E., \& Tarr, M. J. (2009). Figure-ground assignment to a translating contour: A preference for advancing vs. receding motion. Journal of Vision, 9(5), Article 27. doi:10.1167/9.5.27

Baylis, G. C., \& Driver, J. (1994). Parallel computation of symmetry but not repetition within visual shapes. Visual Cognition, 1, 377-400. doi: 10.1080/13506289408401715

Baylis, G. C., \& Driver, J. (1995). Obligatory edge assignment in vision: The role of figure and part segmentation in symmetry detection. Journal of Experimental Psychology: Human Perception and Performance, 21, 1323-1342. doi:10.1037/0096-1523.21.6.1323

Beck, D. M., \& Palmer, S. E. (2002). Top-down influences on perceptual grouping. Journal of Experimental Psychology: Human Perception and Performance, 28, 1071-1084. doi:10.1037/0096-1523.28.5.1071

Beck, J. (Ed.). (1982). Organization and representation in vision. Hillsdale, NJ: Erlbaum.

Bertamini, M. (2006). Who owns the contour of a visual hole? Perception, 35, 883-894. doi:10.1068/p5496

Bertamini, M., \& Hulleman, J. (2006). Amodal completion and visual holes (static and moving). Acta Psychologica, 123, 55-72. doi:10.1016/ j.actpsy.2006.04.006
Bhatt, R. S., \& Quinn, P. C. (2011). How does learning impact development in infancy? The case of perceptual organization. Infancy, 16, 2-38. doi:10.1111/j.1532-7078.2010.00048.x

Biederman, I. (1987). Recognition-by-components: A theory of human image understanding. Psychological Review, 94, 115-147. doi:10.1037/ 0033-295X.94.2.115

Binford, T. (1981). Inferring surfaces from images. Artificial Intelligence, 17, 205-244. doi:10.1016/0004-3702(81)90025-4

Blum, H. (1973). Biological shape and visual science (Part I). Journal of Theoretical Biology, 38, 205-287. doi:10.1016/0022-5193(73)90175-6

Bravais, A. (1949). Crystallographic studies: On the systems formed by points regularly distributed on a plane or in space (A. J. Shaler, Trans.) New York, NY: Crystallographic Society of America. (Original work published 1850)

Bregman, A. S. (1981). Asking the "what for" question in auditory perception. In M. Kubovy \& J. R. Pomerantz (Eds.), Perceptual organization (pp. 99-118). Hillsdale, NJ: Erlbaum.

Bregman, A. S. (1990). Auditory scene analysis: The perceptual organization of sound. Cambridge, MA: Bradford/MIT Press.

Brincat, S. L., \& Connor, C. E. (2004). Underlying principles of visual shape selectivity in posterior inferotemporal cortex. Nature Neuroscience, 7, 880-886. doi:10.1038/nn1278

Brincat, S. L., \& Connor, C. E. (2006). Dynamic shape synthesis in posterior inferotemporal cortex. Neuron, 49, 17-24. doi:10.1016/ j.neuron.2005.11.026

Brown, J. F., \& Voth, A. C. (1937). The path of seen movement as a function of the vector-field. American Journal of Psychology, 49, 543563. doi:10.2307/1416380

Burge, J., Fowlkes, C., \& Banks, M. S. (2010). Natural-scene statistics predict how the figure-ground cue of convexity affects human depth perception. Journal of Neuroscience, 30, 7269-7280. doi:10.1523/ JNEUROSCI.5551-09.2010

Burge, J., Peterson, M. A., \& Palmer, S. E. (2005). Ordinal configural cues combine with metric disparity in depth perception. Journal of Vision, 5(6), Article 5. doi:10.1167/5.6.5

Burt, P., \& Sperling, G. (1981). Time, distance, and feature trade-offs in visual apparent motion. Psychological Review, 88, 171-195. doi: 10.1037/0033-295X.88.2.171

Canny, J. F. (1983). Finding edges and lines in images (Unpublished master's thesis). MIT Artificial Intelligence Laboratory, Cambridge, MA.

Carandini, M., Demb, J. B., Mante, V., Tolhurst, D. J., Dan, Y., Olshausen, B. A., ... Rust, N. C. (2005). Do we know what the early visual system does? Journal of Neuroscience, 25, 10577-10597. doi:10.1523/ JNEUROSCI.3726-05.2005

Casco, C. (1990). The relationship between visual persistence and event perception in bistable motion display. Perception, 19, 437-445. doi: $10.1068 / \mathrm{p} 190437$

Cavanaugh, J. R., Bair, W., \& Movshon, J. A. (2002). Nature and interaction of signals from the receptive field center and surround in macaque V1 neurons. Journal of Neurophysiology, 88, 2530-2546. doi:10.1152/ jn.00692.2001

Claessens, P. M. E., \& Wagemans, J. (2005). Perceptual grouping in Gabor lattices: Proximity and alignment. Perception \& Psychophysics, 67, 1446-1459, doi:10.3758/BF03193649

Claessens, P. M. E., \& Wagemans, J. (2008). A Bayesian framework for cue integration in multistable grouping: Proximity, collinearity, and orientation priors in zigzag lattices. Journal of Vision, 8(7), Article 33. doi:10.1167/8.7.33

Cohen, E. H., \& Singh, M. (2007). Geometric determinants of shape segmentation: Tests using segment identification. Vision Research, 47, 2825-2840. doi:10.1016/j.visres.2007.06.021

Coren, S. (1972). Subjective contours and apparent depth. Psychological Review, 79, 359-367. doi:10.1037/h0032940 
Craft, E., Schütze, H., Niebur, E., \& von der Heydt, R. (2007). A neural model of figure-ground organization. Journal of Neurophysiology, 97, 4310-4326. doi: 10.1152/jn.00203.2007

Dawson, M. R. W., Nevin-Meadows, N., \& Wright, R. D. (1994). Polarity matching in the Ternus configuration. Vision Research, 34, 3347-3359. doi:10.1016/0042-6989(94)90069-8

De Winter, J., \& Wagemans, J. (2006). Segmentation of object outlines into parts: A large-scale integrative study. Cognition, 99, 275-325. doi:10.1016/j.cognition.2005.03.004

Driver, J., \& Baylis, G. C. (1996). Edge-assignment and figure-ground segmentation in short-term visual masking. Cognitive Psychology, 31, 248-306. doi:10.1006/cogp.1996.0018

Driver, J., Baylis, G. C., \& Rafal, R. D. (1992, November 5). Preserved figure-ground segregation and symmetry perception in visual neglect. Nature, 360, 73-75. doi:10.1038/360073a0

Duncan, R. O., Albright, T. D., \& Stoner, G. R. (2000). Occlusion and the interpretation of visual motion: Perceptual and neuronal effects of context. Journal of Neuroscience, 20, 5885-5897.

Duncker, K. (1929). Über induzierte Bewegung (Ein Beitrag zur Theorie optisch wahrgenommener Bewegung) [Concerning induced movement (Contribution to the theory of visually perceived movement)]. Psychologische Forschung, 12, 180-259. doi:10.1007/BF02409210

Elder, J., \& Zucker, S. W. (1993). The effect of contour closure on the rapid discrimination of two-dimensional shapes. Vision Research, 33, 981-991. doi:10.1016/0042-6989(93)90080-G

Elder, J., \& Zucker, S. W. (1994). A measure of closure. Vision Research, 34, 3361-3369. doi:10.1016/0042-6989(94)90070-1

Elder, J. H., \& Goldberg, R. M. (2002). Ecological statistics of Gestalt laws for the perceptual organization of contours. Journal of Vision, 2(4), Article 5. doi:10.1167/2.4.5

Elder, J. H., Krupnik, A., \& Johnston, L. A. (2003). Contour grouping with prior models. IEEE Transactions on Pattern Analysis and Machine Intelligence, 25, 661-674. doi:10.1109/TPAMI.2003.1201818

Ellis, W. D. (Ed.). (1938). A source book of Gestalt psychology. London, England: Routledge \& Kegan Paul. doi:10.1037/11496-000

Epstein, W. (1988). Has the time come to rehabilitate Gestalt theory? Psychological Research, 50, 2-6. doi:10.1007/BF00309403

Epstein, W., \& DeShazo, D. (1961). Recency as a function of perceptual oscillation. American Journal of Psychology, 74, 215-223. doi:10.2307/ 1419406

Estrada, F. J., \& Elder, J. H. (2006). Multi-scale contour extraction based on natural image statistics. In C. Schmid, S. Soatto, \& C. Tomasi (Eds.), Proceedings of the IEEE Conference on Computer Vision and Pattern Recognition Workshop (CVPRW'06) (Article 183, pp. 1-8). New York, NY: IEEE Computer Society.

Fantoni, C., Bertamini, M., \& Gerbino, W. (2005). Contour curvature polarity and surface interpolation. Vision Research, 45, 1047-1062. doi:10.1016/j.visres.2004.10.023

Fantoni, C., \& Gerbino, W. (2003). Contour interpolation by vector-field combination. Journal of Vision, 3(4), Article 4. doi:10.1167/3.4.4

Farid, H. (2002). Temporal synchrony in perceptual grouping: A critique. Trends in Cognitive Sciences, 6, 284-288. doi:10.1016/S13646613(02)01927-7

Farid, H., \& Adelson, E. H. (2001). Synchrony does not promote grouping in temporally structured displays. Nature Neuroscience, 4, 875-876. doi:10.1038/nn0901-875

Feldman, J. (2007). Formation of visual "objects" in the early computation of spatial relations. Perception \& Psychophysics, 69, 816-827. doi: 10.3758/BF03193781

Feldman, J., \& Singh, M. (2006). Bayesian estimation of the shape skeleton. PNAS: Proceedings of the National Academy of Sciences, USA, 103, 18014-18019. doi:10.1073/pnas.0608811103

Field, D. J., Hayes, A., \& Hess, R. F. (1993). Contour integration by the human visual system: Evidence for a local "association field." Vision Research, 33, 173-193. doi:10.1016/0042-6989(93)90156-Q

Field, D. J., Hayes, A., \& Hess, R. F. (2000). The roles of polarity and symmetry in the perceptual grouping of contour fragments. Spatial Vision, 13, 51-66. doi:10.1163/156856800741018

Finkel, L. H., \& Sajda, P. (1992). Object discrimination based on depthfrom-occlusion. Neural Computation, 4, 901-921. doi:10.1162/ neco.1992.4.6.901

Froyen, V., Feldman, J., \& Singh, M. (2010). A Bayesian framework for figure-ground interpretation. In J. Lafferty, C. K. I. Williams, J. ShaweTaylor, R. S. Zemel, \& A. Culotta (Eds.), Advances in neural information processing systems (Vol. 23, pp. 631-639). Vancouver, British Columbia, Canada: Curran Associates.

Fulvio, J. M., \& Singh, M. (2006). Surface geometry influences the shape of illusory contours. Acta Psychologica, 123, 20-40. doi:10.1016/ j.actpsy.2006.02.004

Fulvio, J. M., Singh, M., \& Maloney, L. T. (2008). Precision and consistency of contour interpolation. Vision Research, 48, 831-849. doi: 10.1016/j.visres.2007.12.018

Gallace, A., \& Spence, C. (2011). To what extent do Gestalt grouping principles influence tactile perception? Psychological Bulletin, 137, 538-561. doi:10.1037/a0022335

Geisler, W. S., \& Perry, J. S. (2009). Contour statistics in natural images: Grouping across occlusions. Visual Neuroscience, 26, 109-121. doi: $10.1017 /$ S0952523808080875

Geisler, W. S., Perry, J. S., Super, B. J., \& Gallogly, D. P. (2001). Edge co-occurrence in natural images predicts contour grouping performance. Vision Research, 41, 711-724. doi:10.1016/S0042-6989(00)00277-7

Geisler, W. S., \& Super, B. J. (2000). Perceptual organization of twodimensional patterns. Psychological Review, 107, 677-708. doi: 10.1037/0033-295X.107.4.677

Gepshtein, S., \& Kubovy, M. (2000). The emergence of visual objects in space-time. PNAS: Proceedings of the National Academy of Sciences, USA, 97, 8186-8191. doi:10.1073/pnas.97.14.8186

Gepshtein, S., \& Kubovy, M. (2007). The lawful perception of apparent motion. Journal of Vision, 7(8), Article 9. doi:10.1167/7.8.9

Gerhardstein, P., Kovács, I., Ditre, J., \& Feher, A. (2004). Detection of contour continuity and closure in three-month-olds. Vision Research, 44 2981-2988. doi:10.1016/j.visres.2004.06.023

Ghose, T., \& Palmer, S. E. (2010). Extremal edges versus other principles of figure-ground organization. Journal of Vision, 10(8), Article 3. doi: $10.1167 / 10.8 .3$

Gibson, B. S., \& Peterson, M. A. (1994). Does orientation-independent object recognition precede orientation-dependent recognition? Evidence from a cuing paradigm. Journal of Experimental Psychology: Human Perception and Performance, 20, 299-316. doi:10.1037/00961523.20.2.299

Gibson, J. J. (1971). The legacies of Koffka's Principles. Journal of the History of the Behavioral Sciences, 7, 3-9. doi:10.1002/15206696(197101)7:1<3::AID-JHBS2300070102>3.0.CO;2-1

Gilbert, C. D. (1992). Horizontal integration and cortical dynamics. Neuron, 9, 1-13. doi:10.1016/0896-6273(92)90215-Y

Gilchrist, I. D., Humphreys, G. W., Riddoch, M. J., \& Neumann, H. (1997). Luminance and edge information in grouping: A study using visual search. Journal of Experimental Psychology: Human Perception and Performance, 23, 464-480. doi:10.1037/0096-1523.23.2.464

Gillam, B. J., \& Grove, P. M. (2011). Contour entropy: A new determinant of perceiving ground or a hole. Journal of Experimental Psychology: Human Perception and Performance, 37, 750-757. doi:10.1037/ a0021920

Gillam, B. J., \& Nakayama, K. (2002). Subjective contours at line terminations depend on scene layout analysis, not image processing. Journal of Experimental Psychology: Human Perception and Performance, 28 43-53. doi:10.1037/0096-1523.28.1.43 
Goldreich, D., \& Peterson, M. A. (2012). A Bayesian observer replicates convexity context effects in figure-ground perception. Seeing and Perceiving, 25, 365-395. doi:10.1163/187847612X634445

Gori, S., \& Spillmann, L. (2010). Detection vs. grouping thresholds for elements differing in spacing, size and luminance: An alternative approach towards the psychophysics of Gestalten. Vision Research, 50, 1194-1202. doi:10.1016/j.visres.2010.03.022

Gottschaldt, K. (1926). Über den Einfluß der Erfahrung auf die Wahrnehmung von Figuren. I. Über den Einfluß gehäufter Einprägung von Figuren auf ihre Sichtbarkeit in umfassenden Konfigurationen [About the influence of experience on the perception of figures]. Psychologische Forschung, 8, 261-317. doi:10.1007/BF02411523

Gray, C. M., \& Singer, W. (1989). Stimulus-specific neuronal oscillations in orientation columns of cat visual cortex. PNAS: Proceedings of the National Academy of Sciences, USA, 86, 1698-1702. doi:10.1073/ pnas.86.5.1698

Gregory, R. L. (1972, July 7). Cognitive contours. Nature, 238, 51-52. doi: $10.1038 / 238051 \mathrm{a} 0$

Gregory, R. L. (1987). Illusory contours and occluding surfaces. In S. Petry \& G. E. Meyer (Eds.), Perception of illusory contours (pp. 81-89). New York, NY: Springer. doi:10.1007/978-1-4612-4760-9_9

Gregory, R. L., \& Harris, J. (1974). Illusory contours and stereo depth. Perception \& Psychophysics, 15, 411-416. doi:10.3758/BF03199279

Grossberg, S. (1994). 3-D vision and figure-ground separation by visual cortex. Perception \& Psychophysics, 55, 48-121. doi:10.3758/ BF03206880

Grossberg, S., \& Mingolla, E. (1985). Neural dynamics of form perception: Boundary completion, illusory figures, and neon color spreading. Psychological Review, 92, 173-211. doi:10.1037/0033-295X.92.2.173

Grünbaum, B., \& Shephard, G. C. (1987). Tilings and patterns: An introduction. New York, NY: Freeman.

Guttman, S. E., \& Kellman, P. J. (2004). Contour interpolation revealed by a dot localization paradigm. Vision Research, 44, 1799-1815. doi: 10.1016/j.visres.2004.02.008

Hadad, B., \& Kimchi, R. (2006). Developmental trends in utilizing perceptual closure for grouping of shape: Effects of spatial proximity and collinearity. Perception \& Psychophysics, 68, 1264-1273. doi:10.3758/ BF03193726

Hadad, B., Maurer, D., \& Lewis, T. L. (2010a). The development of contour interpolation: Evidence from subjective contours. Journal of Experimental Child Psychology, 106, 163-176. doi:10.1016/j.jecp .2010 .02 .003

Hadad, B., Maurer, D., \& Lewis, T. L. (2010b). The effects of spatial proximity and collinearity on contour integration in adults and children. Vision Research, 50, 772-778. doi:10.1016/j.visres.2010.01.021

Harrower, M. R. (1936). Some factors determining figure-ground articulation. British Journal of Psychology, 26, 407-424.

Hartmann, G. W. (1935). Gestalt psychology: A survey of facts and principles. New York, NY: Ronald Press. doi:10.1037/11497-000

Hatfield, G., \& Epstein, W. (1985). The status of the minimum principle in the theoretical analysis of visual perception. Psychological Bulletin, 97, 155-186. doi:10.1037/0033-2909.97.2.155

He, Z. J., \& Ooi, T. L. (1999). Perceptual organization of apparent motion in the Ternus display. Perception, 28, 877-892. doi:10.1068/p2941

Heitger, F., Rosenthaler, L., von der Heydt, R., Peterhans, E., \& Kübler, O. (1992). Simulation of neural contour mechanisms: From simple to end-stopped cells. Vision Research, 32, 963-981. doi:10.1016/00426989(92)90039-L

Heitger, F., von der Heydt, R., Peterhans, E., Rosenthaler, L., \& Kübler, O. (1998). Simulation of neural contour mechanisms: Representing anomalous contours. Image and Vision Computing, 16, 407-421. doi: 10.1016/S0262-8856(97)00083-8

Helson, H. (1933). The fundamental propositions of Gestalt psychology. Psychological Review, 40, 13-32. doi:10.1037/h0074375
Hochberg, J., \& Hardy, D. (1960). Brightness and proximity factors in grouping. Perceptual and Motor Skills, 10, 22. doi:10.2466/ pms.1960.10.1.22

Hochberg, J., \& McAlister, E. (1953). A quantitative approach to figural "goodness." Journal of Experimental Psychology, 46, 361-364. doi: 10.1037/h0055809

Hochberg, J., \& Silverstein, A. (1956). A quantitative index of stimulussimilarity: Proximity versus differences in brightness. American Journal of Psychology, 69, 456-458. doi:10.2307/1419052

Hochstein, S., \& Ahissar, M. (2002). View from the top: Hierarchies and reverse hierarchies in the visual system. Neuron, 36, 791-804. doi: 10.1016/S0896-6273(02)01091-7

Hoffman, D. D., \& Richards, W. A. (1984). Parts of recognition. Cognition, 18, 65-96. doi:10.1016/0010-0277(84)90022-2

Hoffman, D. D., \& Singh, M. (1997). Salience of visual parts. Cognition, 63, 29-78. doi:10.1016/S0010-0277(96)00791-3

Hopfield, J. J. (1982). Neural networks and physical systems with emergent collective computational abilities. PNAS: Proceedings of the National Academy of Sciences, USA, 79, 2554-2558. doi:10.1073/ pnas.79.8.2554

Hsiao, H. H. (1928). A suggestive review of Gestalt theory. Psychological Review, 35, 280-297. doi:10.1037/h0072380

Hubel, D. H., \& Wiesel, T. N. (1968). Receptive fields and functional architecture of monkey striate cortex. Journal of Physiology, 195, 215243.

Huggins, P. S., \& Zucker, S. W. (2001). Folds and cuts: How shading flows into edges. In Proceedings of the 8th IEEE International Conference on Computer Vision (Vol. 2, pp. 153-158). Los Alamitos, CA: IEEE Computer Society.

Hulleman, J., \& Humphreys, G. W. (2004). Is there an assignment of top and bottom during symmetry perception? Perception, 33, 615-620. doi:10.1068/p5092

Humphreys, G. W., \& Riddoch, M. J. (1993). Interactions between object and space systems revealed through neuropsychology. In D. E. Meyer \& S. Kornblum (Eds.), Attention and Performance (Vol. 24, pp. 183-218). Cambridge, MA: MIT Press.

Ito, M., \& Gilbert, C. D. (1999). Attention modulates contextual influences in the primary visual cortex of alert monkeys. Neuron, 22, 593-604 doi:10.1016/S0896-6273(00)80713-8

Jacobs, D. W. (1996). Robust and efficient detection of salient convex groups. IEEE Transactions on Pattern Analysis and Machine Intelligence, 18, 23-37. doi:10.1109/34.476008

Jacobs, D. W. (2003). What makes viewpoint-invariant properties perceptually salient? Journal of the Optical Society of America A: Optics, Image Science, and Vision, 20, 1304-1320. doi:10.1364/ JOSAA.20.001304

Jehee, J. F. M., Lamme, V. A. F., \& Roelfsema, P. R. (2007). Boundary assignment in a recurrent network architecture. Vision Research, 47, 1153-1165. doi:10.1016/j.visres.2006.12.018

Kanizsa, G. (1954). Alcune osservazioni sull' effetto Musatti [Some observations on color assimilation]. Archivio di Psicologia Neurologia e Psichiatria, 15, 265-271.

Kanizsa, G. (1955a). Condizioni ed effetti della trasparenza fenomenica [Phenomenal transparency]. Rivista di Psicologia, 49, 3-18.

Kanizsa, G. (1955b). Margini quasi-percettivi in campi con stimolazione omogenea [Quasi-perceptual margins in homogeneously stimulated fields]. Rivista di Psicologia, 49, 7-30.

Kanizsa, G. (1976, April). Subjective contours. Scientific American, 234(4), 48-52. doi:10.1038/scientificamerican0476-48

Kanizsa, G. (1979). Organization in vision: Essays on Gestalt psychology. New York, NY: Praeger Publishers.

Kanizsa, G., \& Gerbino, W. (1976). Convexity and symmetry in figureground organization. In M. Henle (Ed.), Art and artefacts (pp. 25-32) New York, NY: Springer. 
Kellman, P. J., Garrigan, P., Shipley, T. F., \& Keane, B. P. (2007). Interpolation processes in object perception: Reply to Anderson (2007). Psychological Review, 114, 488-502. doi:10.1037/0033295X.114.2.488

Kellman, P. J., \& Shipley, T. F. (1991). A theory of visual interpolation in object perception. Cognitive Psychology, 23, 141-221. doi:10.1016/ 0010-0285(91)90009-D

Kienker, P. K., Sejnowski, T. J., Hinton, G. E., \& Schumacher, L. E. (1986). Separating figure from ground with a parallel network. Perception, 15, 197-216. doi:10.1068/p150197

Kimchi, R. (2000). The perceptual organization of visual objects: A microgenetic analysis. Vision Research, 40, 1333-1347. doi:10.1016/ S0042-6989(00)00027-4

Kimchi, R., Behrman, M., \& Olson, C. R. (Eds.). (2003). Perceptual organization in vision: Behavioral and neural perspectives. Mahwah, NJ: Erlbaum.

Kimchi, R., \& Hadad, B. (2002). Influence of past experience on perceptual grouping. Psychological Science, 13, 41-47. doi:10.1111/14679280.00407

Kimchi, R., Hadad, B., Behrmann, M., \& Palmer, S. E. (2005). Microgenesis and ontogenesis of perceptual organization: Evidence from global and local processing of hierarchical patterns. Psychological Science, 16, 282-290. doi:10.1111/j.0956-7976.2005.01529.x

Kimchi, R., \& Peterson, M. A. (2008). Figure-ground segmentation can occur without attention. Psychological Science, 19, 660-668. doi: 10.1111/j.1467-9280.2008.02140.x

King, D. B., \& Wertheimer, M. (2005). Max Wertheimer \& Gestalt theory. New Brunswick, NJ: Transaction Publishers.

Klapp, S. T., \& Jagacinski, R. J. (2011). Gestalt principles in the control of motor action. Psychological Bulletin, 137, 443-462. doi:10.1037/ a0022361

Koffka, K. (1922). Perception: An introduction to the "Gestalt-Theorie." Psychological Bulletin, 19, 531-585. doi:10.1037/h0072422

Koffka, K. (1935). Principles of Gestalt psychology. London, England: Lund Humphries.

Kogo, N., Strecha, C., Van Gool, L., \& Wagemans, J. (2010). Surface construction by a 2-D differentiation-integration process: A neurocomputational model for perceived border ownership, depth, and lightness in Kanizsa figures. Psychological Review, 117, 406-439. doi:10.1037/ a0019076

Köhler, W. (1920). Die physischen Gestalten in Ruhe und im stationären Zustand. Eine natur-philosophische Untersuchung [The physical Gestalten at rest and in steady state]. Braunschweig, Germany: Vieweg und Sohn.

Köhler, W. (1938). Physical Gestalten. In W. D. Ellis (Ed.), A source book of Gestalt psychology (pp. 17-54). London, England: Routledge \& Kegan Paul. (Original work published 1920)

Köhler, W. (1940). Dynamics in psychology. New York, NY: Liveright.

Köhler, W. (1965). Unsolved problems in the field of figural after-effects. Psychological Record, 15, 63-83.

Köhler, W., \& Held, R. (1949, October 21). The cortical correlate of pattern vision. Science, 110, 414-419. doi:10.1126/science .110 .2860 .414

Köhler, W., \& Wallach, H. (1944). Figural after-effects: An investigation of visual processes. Proceedings of the American Philosophical Society, $88,269-357$.

Korte, A. (1915). Kinematoskopische Untersuchungen [Kinematoscopic investigations]. Zeitschrift für Psychologie, 72, 194-296.

Kourtzi, Z., Tolias, A. S., Altmann, C. F., Augath, M., \& Logothetis, N. K. (2003). Integration of local features into global shapes: Monkey and human fMRI studies. Neuron, 37, 333-346. doi:10.1016/S08966273(02)01174-1

Kovács, I. (2000). Human development of perceptual organization. Vision Research, 40, 1301-1310. doi:10.1016/S0042-6989(00)00055-9
Kovács, I., \& Julesz, B. (1993). A closed curve is much more than an incomplete one: Effect of closure in figure-ground segmentation. PNAS: Proceedings of the National Academy of Sciences, USA, 90, 7495-7497. doi:10.1073/pnas.90.16.7495

Kramer, P., \& Yantis, S. (1997). Perceptual grouping in space and time: Evidence from the Ternus display. Perception \& Psychophysics, 59, 87-99. doi:10.3758/BF03206851

Krantz, D. H., Luce, R. D., Suppes, P., \& Tversky, A. (1971). Foundations of measurement: Vol. I. Additive and polynomial representations. New York, NY: Academic Press.

Krüger, N. (1998). Collinearity and parallelism are statistically significant second order relations of complex cell responses. Neural Processing Letters, 8, 117-129. doi:10.1023/A:1009688428205

Kubovy, M. (1994). The perceptual organization of dot lattices. Psychonomic Bulletin \& Review, 1, 182-190. doi:10.3758/BF03200772

Kubovy, M., Holcombe, A. O., \& Wagemans, J. (1998). On the lawfulness of grouping by proximity. Cognitive Psychology, 35, 71-98. doi: 10.1006/cogp.1997.0673

Kubovy, M., \& Pomerantz, J. R. (Eds.). (1981). Perceptual organization. Hillsdale, NJ: Erlbaum.

Kubovy, M., \& van den Berg, M. (2008). The whole is equal to the sum of its parts: A probabilistic model of grouping by proximity and similarity in regular patterns. Psychological Review, 115, 131-154. doi:10.1037/ 0033-295X.115.1.131

Kubovy, M., \& Wagemans, J. (1995). Grouping by proximity and multistability in dot lattices: A quantitative Gestalt theory. Psychological Science, 6, 225-234. doi:10.1111/j.1467-9280.1995.tb00597.x

Lamme, V. A. F. (1995). The neurophysiology of figure-ground segregation in primary visual cortex. Journal of Neuroscience, 15, 1605-1615.

Lashley, K. S., Chow, K. L., \& Semmes, J. (1951). An examination of the electrical field theory of cerebral integration. Psychological Review, 58, 123-136. doi:10.1037/h0056603

Lee, S.-H., \& Blake, R. (1999, May 14). Visual form created solely from temporal structure. Science, 284, 1165-1168. doi:10.1126/science .284 .5417 .1165

Lee, T. S., Mumford, D., Romero, R., \& Lamme, V. A. F. (1998). The role of the primary visual cortex in higher level vision. Vision Research, 38, 2429-2454. doi:10.1016/S0042-6989(97)00464-1

Leeuwenberg, E. L. J. (1969). Quantitative specification of information in sequential patterns. Psychological Review, 76, 216-220. doi:10.1037/ h0027285

Leeuwenberg, E. L. J. (1971). A perceptual coding language for visual and auditory patterns. American Journal of Psychology, 84, 307-349. doi: $10.2307 / 1420464$

Levitt, J. B., \& Lund, J. S. (2002). The spatial extent over which neurons in macaque striate cortex pool visual signals. Visual Neuroscience, 19, 439-452. doi:10.1017/S0952523802194065

Leyton, M. (1989). Inferring causal history from shape. Cognitive Science, $13,357-387$.

$\mathrm{Li}, \mathrm{Z}$. (1998). A neural model of contour integration in the primary visual cortex. Neural Computation, 10, 903-940. doi:10.1162/089976698300017557

Liu, Z., Jacobs, D. W., \& Basri, R. (1999). The role of convexity in perceptual completion: Beyond good continuation. Vision Research, 39, 4244-4257. doi:10.1016/S0042-6989(99)00141-8

Lowe, D. G. (1985). Perceptual organization and visual recognition. Boston, MA: Kluwer. doi:10.1007/978-1-4613-2551-2

Machilsen, B., Pauwels, M., \& Wagemans, J. (2009). The role of vertical mirror symmetry in visual shape detection. Journal of Vision, 9(12), Article 11. doi:10.1167/9.12.11

Machilsen, B., \& Wagemans, J. (2011). Integration of contour and surface information in shape detection. Vision Research, 51, 179-186. doi 10.1016/j.visres.2010.11.005

Marr, D. (1982). Vision: A computational investigation into the human 
representation and processing of visual information. New York, NY: Freeman.

Marr, D., \& Nishihara, H. K. (1978). Representation and recognition of the spatial organization of three-dimensional shapes. Proceedings of the Royal Society of London, Series B: Biological Sciences, 200, 269-294. doi:10.1098/rspb.1978.0020

Martin, D. R., Fowlkes, C. C., Tal, D., \& Malik, J. (2001). A database of human segmented natural images and its application to evaluating segmentation algorithms and measuring ecological statistics. In Proceedings of the International Conference on Computer Vision (Vol. 2, pp. 416-423). Los Alamitos, CA: IEEE Computer Society Press.

Metelli, F. (1974, April). The perception of transparency. Scientific American, 230(4), 90-98. doi:10.1038/scientificamerican0474-90

Metzger, W. (1930). Optische Untersuchungen am Ganzfeld. II. Zur Phänomenologie des homogenen Ganzfeldes [Optical investigations of the Ganzfeld: II. Toward the phenomenology of the homogeneous Ganzfeld]. Psychologische Forschung, 13, 6-29. doi:10.1007/BF00406757

Metzger, W. (1934). Beobachtungen über phänomenale Identität [Observations on phenomenal identity]. Psychologische Forschung, 19, 1-60. doi:10.1007/BF02409733

Metzger, W. (1936). Gesetze des Sehens [Laws of seeing]. Frankfurt am Main, Germany: Kramer.

Metzger, W. (1941). Psychologie: Die Entwicklung ihrer Grundannahmen seit der Einführung des Experiments [Psychology: The development of basic principles since the introduction of the experimental method]. Darmstadt, Germany: Verlag von Dr. Dietrich Steinkopff.

Metzger, W. (2006). Laws of seeing (L. Spillmann, M. Wertheimer, \& S. Lehar, Trans.). Cambridge, MA: MIT Press.

Michotte, A. (1963). The perception of causality (T. R. Miles \& E. Miles, Trans.). New York, NY: Basic Books. (Original work published 1946)

Michotte, A., Thinès, G., \& Crabbé, G. (1964). Les compléments amodaux des structures perceptives [Amodal completion of perceptual structures]. Leuven, Belgium: Publications Universitaires de Louvain.

Mihalas, S., Dong, Y., von der Heydt, R., \& Niebur, E. (2011). Mechanisms of perceptual organization provide auto-zoom and autolocalization for attention to objects. PNAS: Proceedings of the National Academy of Sciences, USA, 108, 7583-7588. doi:10.1073/pnas .1014655108

Milner, P. M. (1974). A model for visual shape recognition. Psychological Review, 81, 521-535. doi:10.1037/h0037149

Mitchison, G. J., \& Westheimer, G. (1984). The perception of depth in simple figures. Vision Research, 24, 1063-1073. doi:10.1016/00426989(84)90084-1

Mohan, R., \& Nevatia, R. (1992). Perceptual organization for scene segmentation and description. IEEE Transactions on Pattern Analysis and Machine Intelligence, 14, 616-635. doi:10.1109/34.141553

Mumford, D. (1992). On the computational architecture of the neocortex: II. The role of cortico-cortical loops. Biological Cybernetics, 66, 241251. doi:10.1007/BF00198477

Nakayama, K., Shimojo, S., \& Silverman, G. H. (1989). Stereoscopic depth: Its relation to image segmentation, grouping, and the recognition of occluded objects. Perception, 18, 55-68. doi:10.1068/p180055

Navon, D. (1977). Forest before trees: The precedence of global features in visual perception. Cognitive Psychology, 9, 353-383. doi:10.1016/00100285(77)90012-3

Navon, D. (2011). The effect of recognizability on figure-ground processing: Does it affect parsing or only figure selection? Quarterly Journal of Experimental Psychology, 64, 608-624. doi:10.1080/17470218.2010 .516834

Nelson, R., \& Palmer, S. E. (2001). Of holes and wholes: The perception of surrounded regions. Perception, 30, 1213-1226. doi:10.1068/p3148

Neumann, H., \& Sepp, W. (1999). Recurrent V1-V2 interaction in early visual boundary processing. Biological Cybernetics, 81, 425-444. doi: $10.1007 / \mathrm{s} 004220050573$
Nygård, G. E., Sassi, M., \& Wagemans, J. (2011). The influence of orientation and contrast flicker on contour saliency of outlines of everyday objects. Vision Research, 51, 65-73. doi:10.1016/j.visres 2010.09.032

Nygård, G. E., Van Looy, T., \& Wagemans, J. (2009). The influence of orientation jitter and motion on contour saliency and object identification. Vision Research, 49, 2475-2484. doi:10.1016/j.visres.2009.08.002

Orbison, W. D. (1939). Shape as a function of the vector-field. American Journal of Psychology, 52, 31-45. doi:10.2307/1416658

Oyama, T. (1961). Perceptual grouping as a function of proximity. Perceptual and Motor Skills, 13, 305-306.

Oyama, T., Simizu, M., \& Tozawa, J. (1999). Effects of similarity on apparent motion and perceptual grouping. Perception, 28, 739-748. doi:10.1068/p2799

Palmer, S. E. (1977). Hierarchical structure in perceptual representation. Cognitive Psychology, 9, 441-474. doi:10.1016/0010-0285(77)90016-0

Palmer, S. E. (1992). Common region: A new principle of perceptual organization. Cognitive Psychology, 24, 436-447. doi:10.1016/00100285(92)90014-S

Palmer, S. E. (2002a). Perceptual grouping: It is later than you think. Current Directions in Psychological Science, 11, 101-106. doi:10.1111/ 1467-8721.00178

Palmer, S. E. (2002b). Perceptual organization in vision. In H. Pashler (Ed.), Stevens' handbook of experimental psychology: Vol. 1. Sensation and perception (3rd ed., pp. 177-234). New York, NY: Wiley.

Palmer, S. E. (2003). Perceptual organization and grouping. In R. Kimchi, M. Behrmann, \& C. R. Olson (Eds.), Perceptual organization in vision: Behavioral and neural perspectives (pp. 3-43). Mahwah, NJ: Erlbaum.

Palmer, S. E., \& Beck, D. (2007). The repetition discrimination task: An objective method for studying perceptual grouping. Perception \& Psychophysics, 69, 68-78. doi:10.3758/BF03194454

Palmer, S. E., \& Brooks, J. L. (2008). Edge-region grouping in figureground organization and depth perception. Journal of Experimental Psychology: Human Perception and Performance, 34, 1353-1371. doi: 10.1037/a0012729

Palmer, S. E., Brooks, J. L., \& Nelson, R. (2003). When does grouping happen? Acta Psychologica, 114, 311-330. doi:10.1016/ j.actpsy.2003.06.003

Palmer, S. E., \& Ghose, T. (2008). Extremal edges: A powerful cue to depth perception and figure-ground organization. Psychological Science, 19, 77-84. doi:10.1111/j.1467-9280.2008.02049.x

Palmer, S. E., Neff, J., \& Beck, D. (1996). Late influences on perceptual grouping: Amodal completion. Psychonomic Bulletin \& Review, 3, 7580. doi:10.3758/BF03210743

Palmer, S. E., \& Nelson, R. (2000). Late influences on perceptual grouping: Illusory figures. Perception \& Psychophysics, 62, 1321-1331. doi: 10.3758/BF03212134

Palmer, S. E., \& Rock, I. (1994). Rethinking perceptual organization: The role of uniform connectedness. Psychonomic Bulletin \& Review, 1 , 29-55. doi:10.3758/BF03200760

Panis, S., \& Wagemans, J. (2009). Time-course contingencies in perceptual organization and identification of fragmented object outlines. Journal of Experimental Psychology: Human Perception and Performance, 35, 661-687. doi:10.1037/a0013547

Pantle, A., \& Picciano, L. (1976, August 6). A multistable movement display: Evidence for two separate motion systems in human vision. Science, 193, 500-502. doi:10.1126/science.941023

Parent, P., \& Zucker, S. W. (1989). Trace inference, curvature consistency, and curve detection. IEEE Transactions on Pattern Analysis and Machine Intelligence, 11, 823-839. doi:10.1109/34.31445

Pasupathy, A., \& Connor, C. E. (1999). Responses to contour features in macaque area V4. Journal of Neurophysiology, 82, 2490-2502.

Peterhans, E., \& von der Heydt, R. (1989). Mechanisms of contour per- 
ception in monkey visual cortex: II. Contours bridging gaps. Journal of Neuroscience, 9, 1749-1763.

Peterson, M. A. (1994). The proper placement of uniform connectedness. Psychonomic Bulletin \& Review, 1, 509-514. doi:10.3758/BF03210956

Peterson, M. A., \& Enns, J. T. (2005). The edge complex: Implicit memory for figure assignment in shape perception. Perception \& Psychophysics, 67, 727-740. doi:10.3758/BF03193528

Peterson, M. A., \& Gibson, B. S. (1994a). Must figure-ground organization precede object recognition? An assumption in peril. Psychological Science, 5, 253-259. doi:10.1111/j.1467-9280.1994.tb00622.x

Peterson, M. A., \& Gibson, B. S. (1994b). Object recognition contributions to figure-ground organization: Operations on outlines and subjective contours. Perception \& Psychophysics, 56, 551-564. doi:10.3758/ BF03206951

Peterson, M. A., Harvey, E. M., \& Weidenbacher, H. J. (1991). Shape recognition contributions to figure-ground reversal: Which route counts? Journal of Experimental Psychology: Human Perception and Performance, 17, 1075-1089. doi:10.1037/0096-1523.17.4.1075

Peterson, M. A., \& Lampignano, D. W. (2003). Implicit memory for novel figure-ground displays includes a history of cross-border competition. Journal of Experimental Psychology: Human Perception and Performance, 29, 808-822. doi:10.1037/0096-1523.29.4.808

Peterson, M. A., \& Salvagio, E. (2008). Inhibitory competition in figureground perception: Context and convexity. Journal of Vision, 8(16), Article 4. doi:10.1167/8.16.4

Peterson, M. A., \& Skow, E. (2008). Inhibitory competition between shape properties in figure-ground perception. Journal of Experimental Psychology: Human Perception and Performance, 34, 251-267. doi: 10.1037/0096-1523.34.2.251

Pinna, B. (2010). New Gestalt principles of perceptual organization: An extension from grouping to shape and meaning. Gestalt Theory, 32, $11-78$.

Pomerantz, J. R., \& Kubovy, M. (1986). Theoretical approaches to perceptual organization: Simplicity and likelihood principles. In K. R. Boff, L. Kaufman, \& J. P. Thomas (Eds.), Handbook of perception and human performance (pp. 36-1-36-46). New York, NY: Wiley.

Pomerantz, J. R., Sager, L. C., \& Stoever, R. J. (1977). Perception of wholes and their component parts: Some configurational superiority effects. Journal of Experimental Psychology: Human Perception and Performance, 3, 422-435. doi:10.1037/0096-1523.3.3.422

Qiu, F. T., Sugihara, T., \& von der Heydt, R. (2007). Figure-ground mechanisms provide structure for selective attention. Nature Neuroscience, 10, 1492-1499. doi:10.1038/nn1989

Qiu, F. T., \& von der Heydt, R. (2005). Figure and ground in the visual cortex: V2 combines stereoscopic cues with Gestalt rules. Neuron, 47, 155-166. doi:10.1016/j.neuron.2005.05.028

Qiu, F. T., \& von der Heydt, R. (2007). Neural representation of transparent overlay. Nature Neuroscience, 10, 283-284. doi:10.1038/nn1853

Quinlan, P. T., \& Wilton, R. N. (1998). Grouping by proximity or similarity? Competition between the Gestalt principles in vision. Perception, 27, 417-430. doi:10.1068/p270417

Quinn, P. C., \& Bhatt, R. S. (2005). Learning perceptual organization in infancy. Psychological Science, 16, 511-515. doi:10.1111/j.09567976.2005.01567.x

Quinn, P. C., \& Bhatt, R. S. (2006). Are some Gestalt principles deployed more readily than others during early development? The case of lightness versus form similarity. Journal of Experimental Psychology: Human Perception and Performance, 32, 1221-1230. doi:10.1037/00961523.32.5.1221

Rausch, E. (1937). Über Summativität und Nichtsummativität [On summativity and nonsummativity]. Psychologische Forschung, 21, 209289. doi:10.1007/BF02441207

Rensink, R. A., \& Enns, J. T. (1995). Preemption effects in visual search:
Evidence for low-level grouping. Psychological Review, 102, 101-130. doi:10.1037/0033-295X.102.1.101

Ringach, D. L., \& Shapley, R. (1996). Spatial and temporal properties of illusory contours and amodal boundary completion. Vision Research, 36, 3037-3050. doi:10.1016/0042-6989(96)00062-4

Rock, I., \& Brosgole, L. (1964). Grouping based on phenomenal proximity. Journal of Experimental Psychology, 67, 531-538. doi:10.1037/ h0046557

Rock, I., Nijhawan, R., Palmer, S. E., \& Tudor, L. (1992). Grouping based on phenomenal similarity of achromatic color. Perception, 21, 779-789. doi:10.1068/p210779

Roelfsema, P. R. (2006). Cortical algorithms for perceptual grouping. Annual Review of Neuroscience, 29, 203-227. doi:10.1146/annurev .neuro.29.051605.112939

Roelfsema, P. R., Lamme, V. A. F., \& Spekreijse, H. (1998, September 24). Object-based attention in the primary visual cortex of the macaque monkey. Nature, 395, 376-381. doi:10.1038/26475

Roelfsema, P. R., Lamme, V. A. F., \& Spekreijse, H. (2000). The implementation of visual routines. Vision Research, 40, 1385-1411. doi: 10.1016/S0042-6989(00)00004-3

Roelfsema, P. R., Lamme, V. A. F., Spekreijse, H., \& Bosch, H. (2002). Figure-ground segregation in a recurrent network architecture. Journal of Cognitive Neuroscience, 14, 525-537. doi:10.1162/ 08989290260045756

Roelfsema, P. R., Scholte, H. S., \& Spekreijse, H. (1999). Temporal constraints on the grouping of contour segments into spatially extended objects. Vision Research, 39, 1509-1529. doi:10.1016/S00426989(98)00222-3

Rossi, A. F., Desimone, R., \& Ungerleider, L. G. (2001). Contextual modulation in primary visual cortex of macaques. Journal of Neuroscience, 21, 1698-1709.

Rubin, E. (1915). Synsoplevede Figurer. Studier i psykologisk Analyse Visuell wahrgenommene Figuren. Studien in psychologischer Analyse [Visually perceived figures: Studies in psychological analysis]. Copenhagen, Denmark: Gyldendalske Boghandel.

Rubin, E. (1927). Visuell wahrgenommene wirkliche bewegungen [Visually perceived genuine motions]. Zeitschrift für Psychologie, 103, 354 384.

Rush, G. P. (1937). Visual grouping in relation to age. Archives of Psychology, 31, 1-95.

Rust, N. C., \& DiCarlo, J. J. (2010). Selectivity and tolerance ("invariance") both increase as visual information propagates from cortical area V4 to IT. Journal of Neuroscience, 30, 12978-12995. doi:10.1523/ JNEUROSCI.0179-10.2010

Sagara, M., \& Oyama, T. (1957). Experimental studies on figural aftereffects in Japan. Psychological Bulletin, 54, 327-338. doi:10.1037/ h0048995

Sajda, P., \& Finkel, L. H. (1995). Intermediate-level visual representations and the construction of surface perception. Journal of Cognitive Neuroscience, 7, 267-291. doi:10.1162/jocn.1995.7.2.267

Sasaki, Y. (2007). Processing local signals into global patterns. Current Opinion in Neurobiology, 17, 132-139. doi:10.1016/j.conb.2007.03.003

Sassi, M., Vancleef, K., Machilsen, B., Panis, S., \& Wagemans, J. (2010). Identification of everyday objects on the basis of Gaborized outline versions. i-Perception, 1, 121-142. doi:10.1068/i0384

Schulz, M. F., \& Sanocki, T. (2003). Time course of perceptual grouping by color. Psychological Science, 14, 26-30. doi:10.1111/14679280.01414

Schumann, F. (1900). Beiträge zur Analyse der Gesichtswahrnehmungen. I. Einige Beobachtungen über die Zusammenfassung von Gesichtseindrücken zu Einheiten [Contributions to the analysis of visual perception I. Some observations on the combination of visual impressions into units]. Zeitschrift für Psychologie und Physiologie der Sinnesorgane, 23 , 1-32. 
Sejnowski, T. J., \& Hinton, G. E. (1987). Separating figure from ground with a Boltzmann machine. In M. A. Arbib \& A. Hanson (Eds.), Vision, brain, and cooperative computation (pp. 703-724). Cambridge, MA: MIT Press.

Sekuler, A. B., \& Bennett, P. J. (2001). Generalized common fate: Grouping by common luminance changes. Psychological Science, 12, 437444. doi:10.1111/1467-9280.00382

Sekuler, R. (1996). Motion perception: A modern view of Wertheimer's 1912 monograph. Perception, 25, 1243-1258. doi:10.1068/p251243

Sha'ashua, A., \& Ullman, S. (1988). Structural saliency: The detection of globally salient structures using a locally connected network. In Proceedings of the Second International Conference on Computer Vision (pp. 321-327). Los Alamitos, CA: IEEE. doi:10.1109/CCV.1988 .590008

Shadlen, M. N., \& Movshon, J. A. (1999). Synchrony unbound: A critical evaluation of the temporal binding hypothesis. Neuron, 24, 67-77. doi:10.1016/S0896-6273(00)80822-3

Shimojo, S., Silverman, G. H., \& Nakayama, K. (1989). Occlusion and the solution to the aperture problem for motion. Vision Research, 29, 619626. doi:10.1016/0042-6989(89)90047-3

Sigman, M., Cecchi, G. A., Gilbert, C. D., \& Magnasco, M. O. (2001). On a common circle: Natural scenes and Gestalt rules. PNAS: Proceedings of the National Academy of Sciences, USA, 98, 1935-1940. doi:10.1073/ pnas.031571498

Singer, W., \& Gray, C. M. (1995). Visual feature integration and the temporal correlation hypothesis. Annual Review of Neuroscience, 18, 555-586. doi:10.1146/annurev.ne.18.030195.003011

Singh, M. (2004). Modal and amodal completion generate different shapes. Psychological Science, 15, 454-459. doi:10.1111/j.09567976.2004.00701.x

Singh, M., \& Fulvio, J. M. (2005). Visual extrapolation of contour geometry. PNAS: Proceedings of the National Academy of Sciences, USA, 102, 939-944. doi:10.1073/pnas.0408444102

Singh, M., \& Fulvio, J. M. (2007). Bayesian contour extrapolation: Geometric determinants of good continuation. Vision Research, 47, 783798. doi:10.1016/j.visres.2006.11.022

Singh, M., \& Hoffman, D. D. (2001). Part-based representations of visual shape and implications for visual cognition. In T. F. Shipley \& P. J. Kellman (Eds.), Advances in psychology: Vol. 130. From fragments to objects-Segmentation and grouping in vision (pp. 401-459). New York, NY: Elsevier Science. doi:10.1016/S0166-4115(01)80033-9

Sinico, M. (2008). Demonstration in experimental phenomenology: How to bring out perceptual laws. Theory \& Psychology, 18, 853-863. doi:10.1177/0959354308097261

Spehar, B. (2002). The role of contrast polarity in perceptual closure. Vision Research, 42, 343-350. doi:10.1016/S0042-6989(01)00287-5

Sperry, R. W., Miner, N., \& Myers, R. E. (1955). Visual pattern perception following subpial slicing and tantalum wire implantations in the visual cortex. Journal of Comparative and Physiological Psychology, 48, 5058. doi:10.1037/h0043456

Spillmann, L. (1999). From elements to perception: Local and global processing in visual neurons. Perception, 28, 1461-1492. doi:10.1068/ p2763

Spillmann, L. (2009). Phenomenology and neurophysiological correlations: Two approaches to perception research. Vision Research, 49, 1507-1521. doi:10.1016/j.visres.2009.02.022

Stahl, J. S., \& Wang, S. (2008). Globally optimal grouping for symmetric closed boundaries by combining boundary and region information. IEEE Transactions on Pattern Analysis and Machine Intelligence, 30, 395411. doi:10.1109/TPAMI.2007.1186

Steinman, R. M., Pizlo, Z., \& Pizlo, F. J. (2000). Phi is not beta, and why Wertheimer's discovery launched the Gestalt revolution. Vision Research, 40, 2257-2264. doi:10.1016/S0042-6989(00)00086-9

Takeichi, H., Nakazawa, H., Murakami, I., \& Shimojo, S. (1995). The theory of the curvature-constraint line for amodal completion. Perception, 24, 373-389. doi:10.1068/p240373

Ternus, J. (1926). Experimentelle Untersuchungen über phänomenale Identität [The problem of phenomenal identity]. Psychologische Forschung, 7, 81-136.

Treisman, A. M., \& Gelade, G. (1980). A feature-integration theory of attention. Cognitive Psychology, 12, 97-136. doi:10.1016/00100285(80)90005-5

Trujillo, L. T., Allen, J. J. B., Schnyer, D. M., \& Peterson, M. A. (2010) Neurophysiological evidence for the influence of past experience on figure-ground perception. Journal of Vision, 10(2), Article 5. doi: $10.1167 / 10.2 .5$

Tse, P. U. (1999). Complete mergeability and amodal completion. Acta Psychologica, 102, 165-201. doi:10.1016/S0001-6918(99)00027-X

Tversky, T., Geisler, W. S., \& Perry, J. S. (2004). Contour grouping: Closure effects are explained by good continuation and proximity. Vision Research, 44, 2769-2777. doi:10.1016/j.visres.2004.06.011

Ullman, S. (1976). Filling in the gaps: The shape of subjective contours and a model for their generation. Biological Cybernetics, 25, 1-6.

Ullman, S. (1979). The interpretation of visual motion. Cambridge, MA: MIT Press.

van den Berg, M., Kubovy, M., \& Schirillo, J. A. (2011). Grouping by regularity and the perception of illumination. Vision Research, 51, 1360-1371. doi:10.1016/j.visres.2011.04.013

van Lier, R. (1999). Investigating global effects in visual occlusion: From a partly occluded square to a tree-trunk's rear. Acta Psychologica, 102, 203-220. doi:10.1016/S0001-6918(98)00055-9

van Lier, R., van der Helm, P. A., \& Leeuwenberg, E. L. J. (1994) Integrating global and local aspects of visual occlusion. Perception, 23, 883-903. doi:10.1068/p230883

van Lier, R. J., van der Helm, P. A., \& Leeuwenberg, E. L. J. (1995). Competing global and local completions in visual occlusion. Journal of Experimental Psychology: Human Perception and Performance, 21, 571-583. doi:10.1037/0096-1523.21.3.571

Vecera, S. P. (2004). The reference frame of figure-ground assignment. Psychonomic Bulletin \& Review, 11, 909-915. doi:10.3758/ BF03196720

Vecera, S. P., \& Farah, M. J. (1997). Is visual image segmentation a bottom-up or an interactive process? Perception \& Psychophysics, 59, 1280-1296. doi:10.3758/BF03214214

Vecera, S. P., Flevaris, A. V., \& Filapek, J. C. (2004). Exogenous spatial attention influences figure-ground assignment. Psychological Science, 15, 20-26. doi:10.1111/j.0963-7214.2004.01501004.x

Vecera, S. P., \& Palmer, S. E. (2006). Grounding the figure: Contextual effects of depth planes on figure-ground organization. Psychonomic Bulletin \& Review, 13, 563-569. doi:10.3758/BF03193963

Vecera, S. P., Vogel, E. K., \& Woodman, G. F. (2002). Lower region: A new cue for figure-ground assignment. Journal of Experimental Psychology: General, 131, 194-205. doi:10.1037/0096-3445.131.2.194

Vezzani, S., Marino, B. F. M., \& Giora, E. (2012). An early history of the Gestalt factors of organization. Perception, 41, 148-167. doi:10.1068/ p7122

Vickery, T. J., \& Jiang, Y. V. (2009). Associative grouping: Perceptual grouping of shapes by association. Attention, Perception, \& Psychophysics, 71, 896-909. doi:10.3758/APP.71.4.896

von der Heydt, R., Peterhans, E., \& Baumgartner, G. (1984, June 15). Illusory contours and cortical neuron responses. Science, 224, 1260 1262. doi: $10.1126 /$ science. 6539501

von der Malsburg, C. (1981). The correlation theory of brain function (Departmental Technical Report No. 81-2). Göttingen, Germany: MaxPlanck-Institut für Biophysical Chemistry.

von Ehrenfels, C. (1988). On "Gestalt qualities." In B. Smith (Ed. \& Trans.), Foundations of Gestalt theory (pp. 82-117). Munich, Germany: Philosophia Verlag. (Original work published 1890) 
Wagemans, J. (1992). Perceptual use of nonaccidental properties. Canadian Journal of Psychology/Revue canadienne de psychologie, 46, 236279. doi: $10.1037 / \mathrm{h} 0084323$

Wagemans, J. (1993). Skewed symmetry: A nonaccidental property used to perceive visual forms. Journal of Experimental Psychology: Human Perception and Performance, 19, 364-380. doi:10.1037/00961523.19.2.364

Wagemans, J., Feldman, J., Gepshtein, S., Kimchi, R., Pomerantz, J. R., van der Helm, P. A., \& van Leeuwen, C. (2012). A century of Gestalt psychology in visual perception: II. Conceptual and theoretical foundations. Psychological Bulletin, 138, 1218-1252. doi:10.1037/a0029334

Wagemans, J., van Lier, R., \& Scholl, B. J. (2006). Introduction to Michotte's heritage in perception and cognition research. Acta Psychologica, 123, 1-19. doi:10.1016/j.actpsy.2006.06.003

Wallace, J. M., \& Scott-Samuel, N. E. (2007). Spatial versus temporal grouping in a modified Ternus display. Vision Research, 47, 2353-2366. doi:10.1016/j.visres.2007.05.016

Wannig, A., Stanisor, L., \& Roelfsema, P. R. (2011). Automatic spread of attentional response modulation along Gestalt criteria in primary visual cortex. Nature Neuroscience, 14, 1243-1244. doi:10.1038/nn.2910

Wertheimer, M. (1912). Experimentelle Studien über das Sehen von Bewegung [Experimental studies on the seeing of motion]. Zeitschrift für Psychologie, 61, 161-265.

Wertheimer, M. (1922). Untersuchungen zur Lehre von der Gestalt, I: Prinzipielle Bemerkungen [Investigations in Gestalt theory: I. The general theoretical situation]. Psychologische Forschung, 1, 47-58.

Wertheimer, M. (1923). Untersuchungen zur Lehre von der Gestalt, II. [Investigations in Gestalt Theory: II. Laws of organization in perceptual forms]. Psychologische Forschung, 4, 301-350.

Wertheimer, M. (1938a). The general theoretical situation. In W. D. Ellis (Ed.), A source book of Gestalt psychology (pp. 12-16). London, England: Routledge \& Kegan Paul. (Original work published 1922)

Wertheimer, M. (1938b). Gestalt theory. In W. D. Ellis (Ed.), A source book of Gestalt psychology (pp. 1-11). London, England: Routledge \& Kegan Paul. (Original work published 1924)

Wertheimer, M. (1938c). Laws of organization in perceptual forms. In W. D. Ellis (Ed.), A source book of Gestalt psychology (pp. 71-94).
London, England: Routledge \& Kegan Paul. (Original work published 1923)

Wertheimer, M. (1945). Productive thinking. New York, NY: Harper.

Wertheimer, M. (1961). Experimental studies on the seeing of motion. In T. Shipley (Ed.), Classics in psychology (pp. 1032-1089). New York, NY: Philosophical Library. (Original work published 1912)

Westheimer, G. (1999). Gestalt theory reconfigured: Max Wertheimer's anticipation of recent developments on visual neuroscience. Perception, 28, 5-15. doi:10.1068/p2883

Williams, L. R., \& Jacobs, D. W. (1997). Stochastic completion fields: A neural model of illusory contour shape and salience. Neural Computation, 9, 837-858. doi:10.1162/neco.1997.9.4.837

Yen, S. C., \& Finkel, L. H. (1998). Extraction of perceptually salient contours by striate cortical networks. Vision Research, 38, 719-741. doi:10.1016/S0042-6989(97)00197-1

Yin, C., Kellman, P. J., \& Shipley, T. F. (2000). Surface integration influences depth discrimination. Vision Research, 40, 1969-1978. doi: 10.1016/S0042-6989(00)00047-X

Zhang, N. R., \& von der Heydt, R. (2010). Analysis of the context integration mechanisms underlying figure-ground organization in the visual cortex. Journal of Neuroscience, 30, 6482-6496. doi:10.1523/ JNEUROSCI.5168-09.2010

Zhaoping, L. (2005). Border ownership from intracortical interactions in visual area V2. Neuron, 47, 143-153. doi:10.1016/j.neuron.2005.04.005

Zhou, H., Friedman, H. S., \& von der Heydt, R. (2000). Coding of border ownership in monkey visual cortex. Journal of Neuroscience, 20, $6594-$ 6611.

Zhou, J., Tjan, B. S., Zhou, Y., \& Liu, Z. (2008). Better discrimination for illusory than for occluded perceptual completions. Journal of Vision, 8(7), Article 26. doi:10.1167/8.7.26

Zipser, K., Lamme, V. A. F., \& Schiller, P. H. (1996). Contextual modulation in primary visual cortex. Journal of Neuroscience, 16, 73767389.

Received June 27, 2011

Revision received May 6, 2012

Accepted May 11, 2012 